\title{
Magnetic Activity at the Poles of the Sun
}

\author{
Dissertation \\ zur Erlangung des Doktorgrades \\ der Mathematisch-Naturwissenschaftlichen Fakultäten \\ der Georg-August-Universität zu Göttingen
}

vorgelegt von

\section{Julián Blanco Rodríguez \\ aus Salamanca / Spanien}

Göttingen 2008 
D7

Referent: Prof. Dr. F. Kneer

Korreferent: Prof. Dr. W. Kollatschny

Tag der mündlichen Prüfung: 
With magic, you can turn a frog into a prince. With science, you can turn a frog into a Ph.D. and still have the same frog you started with.

Terry Pratchett, Ian Eddington \& Jack Cohen - The Science of Discworld 



\section{Contents}

Contents 5

\begin{tabular}{ll}
\hline Summary & 7
\end{tabular}

1 Introduction 9

1.1 The Sun . . . . . . . . . . . . . . . . . . . . 9

1.2 Aims of the present study . . . . . . . . . . . . . . . . . . 14

$\begin{array}{lll}2 & \text { Spectropolarimetry } & 17\end{array}$

2.1 Spectroscopy . . . . . . . . . . . . . . . . . . . . . . 17

2.2 Polarisation ... . . . . . . . . . . . . . . . . . . . . 18

2.3 Zeeman splitting . . . . . . . . . . . . . . . 21

3 Instrumentation and Observations 27

3.1 Instruments . . . . . . . . . . . . . . . . . . . . . . . . 27

3.1 .1 Kiephenheuer Adaptive Optics System . . . . . . . . . . . . . 29

3.1 .2 "Göttingen" Fabrv-Perot Interferometer . . . . . . . . . . . . . 32

3.1 .3 Tenerife Infrared Polarimeter II $\ldots \ldots \ldots$

3.2 Campaigns $\ldots \ldots \ldots \ldots \ldots \ldots \ldots \ldots \ldots \ldots \ldots$

4 Data Reduction 41

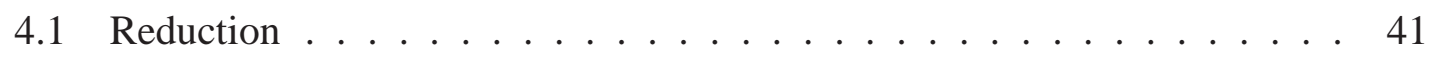

4.2 Speckle reconstruction . . . . . . . . . . . . . . . . . . . 43

4.3 Magnetic field and velocity determination . . . . . . . . . . . . . 49

$\begin{array}{lll}5 & \text { Results } & 57\end{array}$

5.1 Photometric analvsis . . . . . . . . . . . . . . . . . . . . . 59

$5.1 .1 \quad$ Number and size density . . . . . . . . . . . . . . . . . . . . . 59

5.1 .2 Centre-to-limb variation of contrast . . . . . . . . . . . . 63

$5.1 .3 \quad \mathrm{H} \alpha$ analvsis . . . . . . . . . . . . . . . . . . . . . . 65

5.1 .4 Temporal evolution . . . . . . . . . . . . . . . . . . . . . 67

5.2 Magnetic field . . . . . . . . . . . . . . . . . . . . . 68

5.2 .1 Comparison of methods . . . . . . . . . . . . . . . 68

5.2 .2 Polar Faculae . . . . . . . . . . . . . . . . . . . . 70

5.2.2.1 Centre Of Gravity and Weak Field Approximation . . . 70

5.2 .2 .2 Strong Field Regime . . . . . . . . . . . . . . . . . . . 72

5.2 .2 .3 Total magnetic flux in PFe . . . . . . . . . . . . 73 
5.2 .3 Magnetic flux outside $\mathrm{PFe}$. . . . . . . . . . . 76

5.3 Velocity . . . . . . . . . . . . . . . . . 76

$\begin{array}{lll}6 & \text { Conclusions } & 81\end{array}$

\begin{tabular}{ll}
\hline Bibliography & 85
\end{tabular}

\begin{tabular}{ll}
\hline Publications & 91
\end{tabular}

\begin{tabular}{|ll}
\hline Acknowledgements & 93
\end{tabular}

\begin{tabular}{ll}
\hline Curriculum Vitae & 95
\end{tabular} 


\section{Summary}

All activity that takes place on the Sun is triggered and driven by magnetic fields. Thus, the investigation and understanding of the solar magnetic field can shed light on the features observed on the Sun and their evolution. Furthermore, it can also help the analyses of other stars and celestial bodies which possess magnetic fields as well.

One of the most captivating aspects of the solar magnetic field is the so-called activity cycle. The magnetic field on the Sun evolves from poloidal to toroidal and again to poloidal, with polarity reversed to that in the first state, on an approximately 11 years basis. The surface of the Sun during the maximum of activity with predominantly toroidal field is characterised by the appearance of sunspots. This phase of the cycle has been studied in depth for long time.

During the realisation of the present work, the Sun was near a minimum of sunspot activity, i.e. the global magnetic field was mostly poloidal. Therefore, this was the best epoch to study the magnetic activity at the poles of the Sun. The present work has focused on polar faculae $(\mathrm{PFe})$, small-scale, bright magnetic features that appear at the polar caps of the Sun, down to latitudes $|\psi| \approx 60^{\circ}$. From previous studies, PFe are known to possess magnetic fields in the kilo-Gauss range and to have an activity cycle shifted 5-6 years with respect to that of sunspots. This means that their maximum of occurrence happens during the sunspot minimum, the time when the observations for the present study were obtained.

This thesis work analyses the properties of PFe and their relation to the global poloidal field by means of statistical samples. The observations were performed with the "Göttingen" Fabry-Perot interferometric (FPI) spectrometer and with the Tenerife Infrared Polarimeter II (TIP II) attached to the echelle spectrograph of the Vacuum Tower Telescope (VTT) at the Observatorio del Teide / Tenerife, thus allowing to have information on PFe with high spatial resolution (FPI plus speckle reconstruction methods) and with high spectral resolution (TIP II). Furthermore, thanks to the recent upgrade of the FPI providing, among other new improvements, the possibility of quasi-simultaneous observations in different spectral regions, $P F e$ have been observed at two different atmospheric layers: a) The magnetically sensitive iron line Fe I $6173.3 \AA$ was analysed to measure photospheric magnetic fields. b) The chromospheric $\mathrm{H} \alpha$ line was used to trace the penetration of PFe to higher layers, up to the chromosphere. The magnetically very sensitive iron lines at $1.56 \mu \mathrm{m}$ were observed with TIP II, supplying observations in the infrared spectral range for comparison with results from the visible spectral line.

The comparison of infrared and visible lines yields a high consistency in both regimes, with very similar results in all the analyses. The highest differences are larger PF areas and lower strengths of the line of sight component of the magnetic field from TIP II data compared to FPI data. This is caused by the much lower spatial resolution of TIP II. 
PF counting results in a much higher occurrence than observed hitherto. An asymmetry between north and south poles is seen, in the sense that near the north pole more PFe are found than in the south polar cap. The asymmetry being higher from visible observations, it is present in both visible and infrared data. Most of the PFe found at each pole have the same polarity as the global magnetic field, yet a non-negligible amount of PFe possesses opposite polarity.

A long time series of a particular PF, lasting approximately 6 hours, was also observed. Despite the fast evolution of small substructures of the PF in time scales of around 10 seconds, the PF itself (and neighbouring ones) remains as an identifiable structure for the whole duration of the time series.

Apart from the difference mentioned above in the values of the strength of the LOS component, both visible and infrared (crosstalk-free) lines give the same peculiar result: No variation of the field strength towards limb is noticeable.

From extrapolated PF areas and the total field strength of PFe, the total magnetic flux in the polar caps residing in PFe has been measured and compared with previous works. Although harbouring an important amount of flux, PFe cannot account for the total magnetic flux at the poles of the Sun. The magnetic flux found in the FOVs outside $\mathrm{PFe}$ is of the same order of that of PFe.

Velocity analyses, performed over the three different regimes (infrared, visible and $\mathrm{H} \alpha$ ), show a high agreement. PFe present a constant outflow of approximately $0.3 \mathrm{~km} \mathrm{~s}^{-1}$ until the top height of the observations, around $1 \mathrm{Mm}$. From these results, PFe qualify to be the photospheric sources of the fast solar wind. Observations at even higher atmospheric layers are necessary to confirm the continuous outflow and acceleration of material from $\mathrm{PFe}$ to high velocities in the fast solar wind from polar coronal holes. 


\title{
1 Introduction
}

\author{
"Most men, they'll tell you a story straight through. \\ It won't be complicated, but it won't be interesting either." \\ Big Fish (2003)
}

\subsection{The Sun}

Astrophysics is a science that deals with a wide variety and range of matter and phenomena, from energetic particles to planets, comets, stars, galaxies, space and time, their interactions, origins and evolution. Ultimately, it deals with the beginning and ending of everything.

The enormity of the scales in which astrophysics spreads, both in size and distance, together with the limited instrumental capacity, usually only allows small capacity to uncover the tiny details, the trees hidden in the forest. Fortunately, each passing year new instruments, simulations and theories make these details more accessible. Even more fortunate is to have a great example close enough to us.

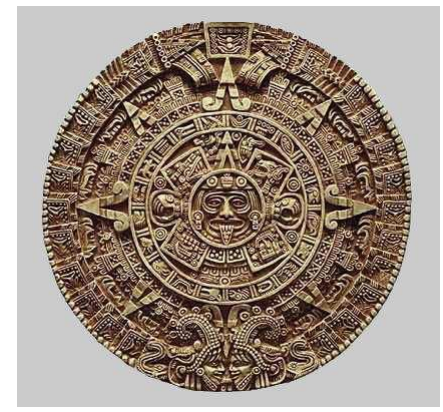

Figure 1.1: Aztec solar calendar.

The Sun has been the centre of legends, religions, calendars (e.g. Fig. 1.1) and life for the human beings since we have records of it. It was even the centre of the universe during a long time. Nowadays many cultural references and traditions related to the Sun still persist. Among them are astrophysical studies, where a whole branch is dedicated to this single star.

On its own, the Sun is no special star. Rather small, with no striking attributes like extreme activity or strong winds, it is located in the main sequence, the area of the Hertzsprung-Russell diagram 1 in which stars are fusing hydrogen in their cores, producing helium - stars spend most of their lifes in this state. Nothing by itself suggests that the Sun should be given so much attention in the analysis of the universe. Only its location. Just by being close to us, makes the Sun a great example, an indispensable element of the whole puzzle. For with it and with the resolving capabilities thanks to the Sun's proximity, we can test and learn about plasmas, neutrinos, stellar magnetism,

\footnotetext{
${ }^{1}$ Known by this name are the diagrams representing magnitude versus spectral type or colour and, in some other cases, luminosity versus temperature. Mostly used for stellar evolution and star cluster studies.
} 
stellar winds, seismic properties of stellar interiors, magnetic reconnection, magnetic interaction of stars with their environment, and so on.

Apart from the Sun, the closest star to Earth is Proxima Centauri at approximately 4.2 light years distance which is about 270000 times farther than the Sun-Earth distance. For a simple comparison, imagine a sunspot observed from Earth as a mole of 1 centimetre diameter on a person's skin at 1 meter distance. Then, searching for a similar spot in Proxima Centauri would mean to detect that 1 centimetre mole at 270 kilometres distance. And sunspots are by far not the smallest features on the Sun.

Thanks to the high spatial resolution achievable by the Sun's closeness to Earth, different observational techniques have been applied with high precision along the years to study the Sun. These techniques have allowed to discern different layers in the solar atmosphere. This distinction is depicted in Fig. 1.2 with the names of the layers as well as some observable features. The surface of the Sun, what is seen when observing the

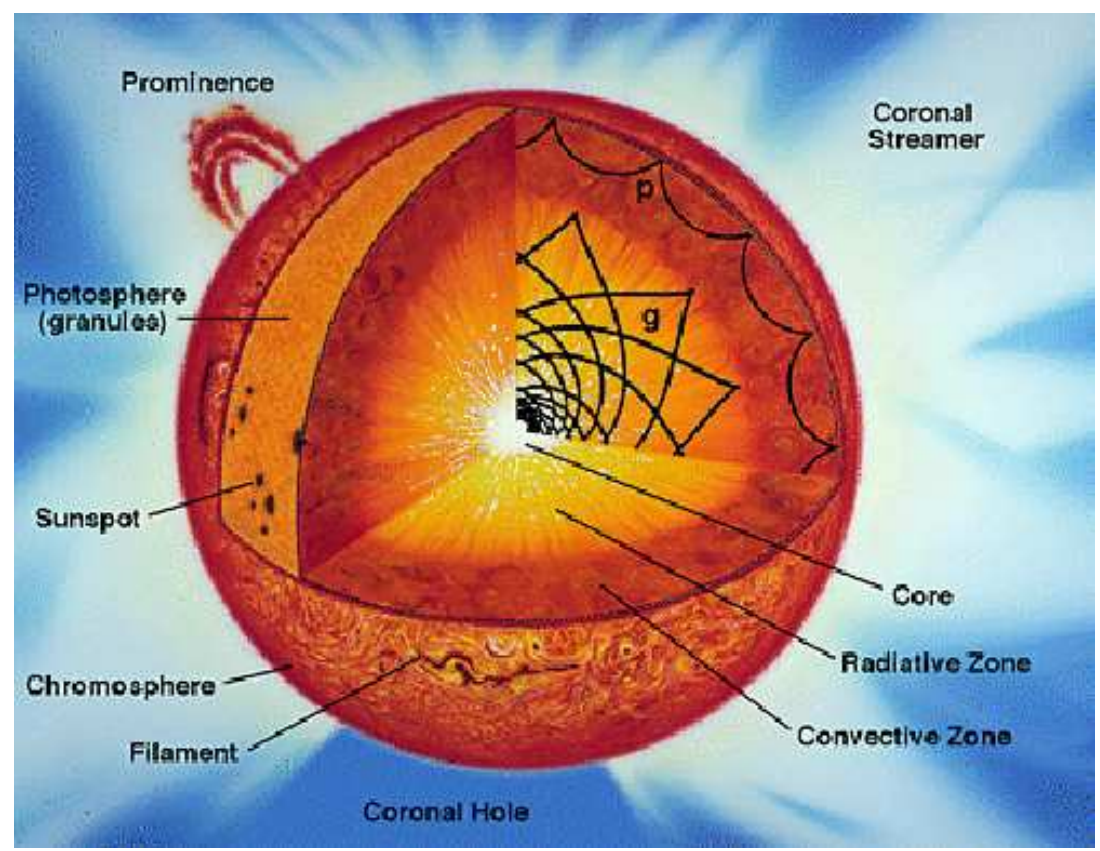

Figure 1.2: Artist's view of the solar atmospheric layers with some characteristic structures and of the solar interior permeated by waves ( $p$ and $g$ ). Image from http://nmp.nasa.gov/st5/SCIENCE/sun.html.

Sun in continuum or in white light, is called photosphere. There, sunspots are visible, as well as the granulation. The atmospheric layers below the photosphere are not accessible to optical observation and the layers above the photosphere are transparent at most wavelengths.

The outer solar layers can be studied by means of filters and coronagraphs (a special telescope in which an occulting disk prevents the scattered light of the very bright photosphere to reach the detector). Photons from these layers can escape from the Sun, while the layers below are optically thick. One way to know about the inner structure of the Sun is through helioseismology. Studying the waves that travel inside the Sun (denoted in Fig. 1.2 as $p$ and $g$ ) in the same manner as do seismic studies on Earth, the solar interior has been probed and subdivided into three areas. In the innermost part, the core, hydro- 
gen atoms are fusing, producing helium and the energy that is ultimately radiated from the Sun. Its radius is $20 \%$ of the total solar radius.

After the core comes the radiative zone. It is named after the mechanism of energy transport dominating in this layer. The most efficient way of transporting the energy generated in the core is by radiation.

The outermost layer of the solar interior is the convective zone, occupying the last $30 \%$ of the solar radius. In this layer the transport of energy by radiation is no longer efficient enough. The energy transport here is mainly performed by convection. Gas parcels move to the surface carrying the energy and release it there. The granules observed in the photosphere are the outermost manifestation from this type of energy transport.

Above the photosphere of approximately $500 \mathrm{~km}$ thickness, the chromosphere extends 2000-4000 km higher up. It is characterised by an outward increase of temperature, on average, from $4000 \mathrm{~K}$ to $10000 \mathrm{~K}$. Also it is very rugged and exhibits many dynamic features.

The last two layers of the solar atmosphere are the transition region and finally the corona. The transition region is a thin layer in which the temperature of the plasma increases rapidly, from the $10000 \mathrm{~K}$ temperature of the upper chromosphere to the coronal temperature of 1 million $\mathrm{K}$ and more. The mechanisms to heat these outer layers represent nowadays one of the most studied unsolved problems about the solar structure.

The corona is a low-density plasma region which extends $13-20$ solar radii outwards. It is the origin of the solar wind which permeates the whole solar system.

One of the most fascinating characteristics of the Sun is its magnetic field. Although the solar magnetic field is not a special case among stars (neither especially strong nor especially fast evolving), the proximity of the Earth to the Sun allows to analyse this magnetic field with high spatial and temporal resolution, as well as in different solar layers. According to the present knowledge, the solar magnetic field is produced and anchored at the base of the convection zone, in the area known as tachocline. From there, the solar magnetic field rises to the solar surface, expands from there to the corona in magnetic loops and drives the solar wind, filling the interplanetary medium until meeting with the interstellar medium. On its way from the interior to far outside, the solar magnetic field affects all matter which it encounters either by just perturbing it or even by confining it and governing its dynamics. At the solar surface and deeper, the magnetic field modifies the normal gas flow, the convection pattern, the travelling of waves, and more, giving rise to so-called "active phenomena" as sunspots, plages, etc. At higher layers in the solar atmosphere, the magnetic field directly controls the behaviour of the gas, dominating its dynamics, as in prominences or the whole corona. Some effects of the magnetic field influence are shown in Fig. 1.2 The mutual influence of magnetic field and gas can be expressed by the number $\beta$, or plasma beta. It represents the ratio of the gas pressure to the pressure of the magnetic field. For example, in the outer solar atmosphere, the density is very low thus the magnetic pressure dominates over the gas pressure, so $\beta$ is small.

A very important characteristic of the solar magnetic field is that it changes periodically in time. Sunspots were known since long time, but it was not until 1843 when Heinrich Schwabe announced a periodic variation of the number of sunspots present on the solar surface. From his own observations during 17 years, he calculated a period of 
around 10 years for this cycle. Since then many studies have been devoted to this solar activity cycle and its implications, both on the Sun and at Earth, being the variation of the sunspot number only one surface effect of the periodic behaviour of the solar magnetic field.

Nowadays, the length of the cycle is established as 11 years on average, with observed durations from 9 to 14 years. The maxima and minima of an activity cycle correspond to the maxima and minima of the sunspot cycle, respectively. So a maximum occurs when the number of sunspots is highest. However, not every solar magnetic phenomenon exhibits the same cyclic behaviour. For example, the polar magnetic fields of the Sun reach their maximum extension over the polar areas during minimum of sunspot activity, whereas during maximum of activity the polarity of the global magnetic field of the Sun undergoes a reversal. This is shown in Fig. 1.3

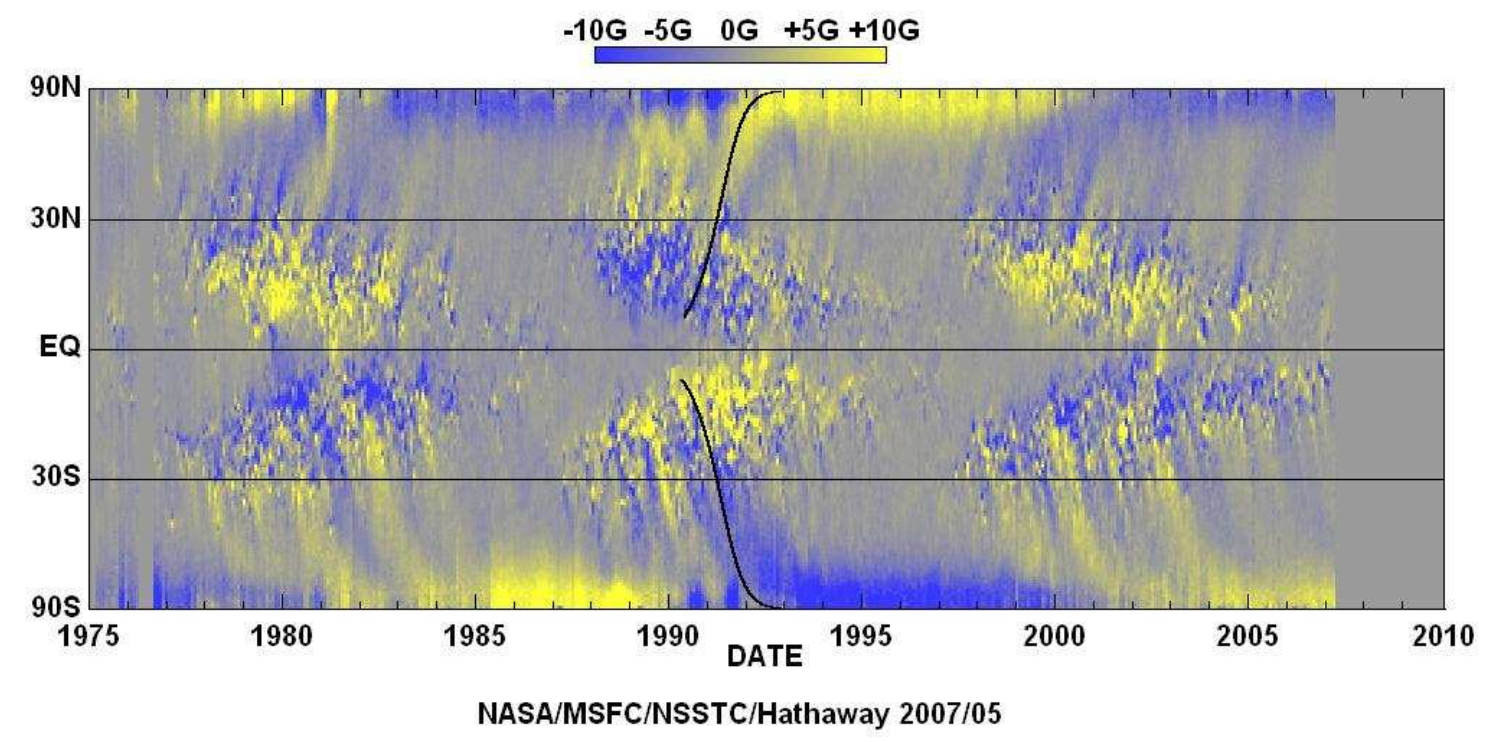

Figure 1.3: Magnetic butterfly diagram.

Figure 1.3 is a magnetic version of the so-called butterfly diagram, named after the shape of the latitude-time occurrence of sunspots, which remind of the wings of butterflies. It consists of sunspot numbers averaged over one solar rotation period for each latitude. In the case of Fig. 1.3 the magnetic flux is depicted instead of number counts. In Fig. 1.3 the so-called activity belts, which are the latitudes of sunspot appearance and correspond to latitudes $|\psi| \leq 40^{\circ}$, are clearly visible. Note also that during the realization of the present work (2005-2007), the Sun was almost at its minimum of activity.

The solar magnetic activity cycle can be considered as a poloidal magnetic field at minimum that turns into a toroidal field at maximum with the peak appearance of sunspots. From there it develops again into a poloidal field with opposite polarity. The mostly accepted mechanism for this behaviour is the $\alpha \Omega$ dynamo, illustrated in Fig. 1.4. Two consecutive effects take place during the activity cycle to regenerate the solar magnetic field. Due to the differential rotation of the Sun - the equator rotates faster than the 


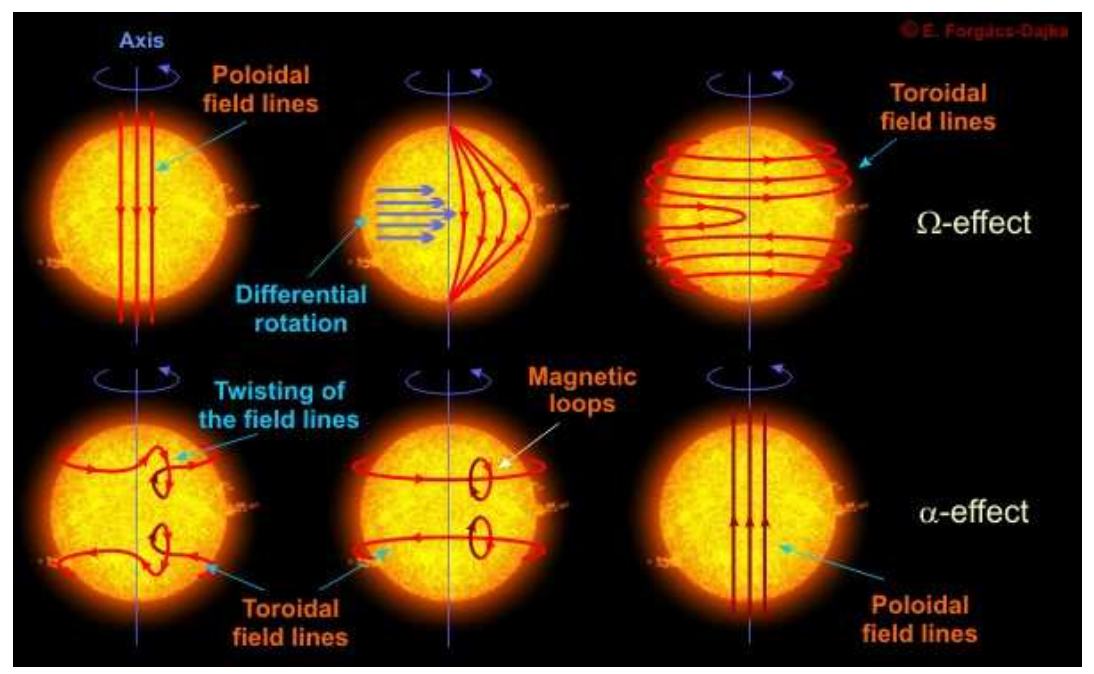

Figure 1.4: Scheme of the $\Omega$ effect (upper) and $\alpha$ effect (lower) on the Sun. CForgacs-Dajka

poles - and because the magnetic field is driven by the plasma in the inner solar layers, the originally poloidal field lines become toroidal field lines. This is the $\Omega$ effect. The $\alpha$ effect is the twisting of the toroidal magnetic field lines, generated as a consequence of the Sun's rotation on the rising tubes of magnetic field. The twist produced by the $\alpha$ effect explains Joy's rule and Hale's rule of magnetic sunspot groups. Joy's rule states that sunspot groups tend to be "tilted", the leading sunspot being closer to the equator than the trailing ones.

During a sunspot maximum, the leading and trailing spots of bipolar groups possess opposite magnetic polarity, e.g. always positive (negative) polarity of the leading spot in the northern (southern) hemisphere. In the subsequent sunspot cycle, the polarities of leading and trailing spots have reversed. This is known as Hale's rule. Thus, the full magnetic cycle is 22 years long. Both these two rules are sketched in Fig. 1.5
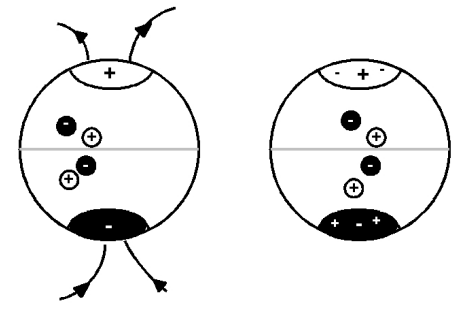

Figure 1.5: Magnetic polarities of the solar poles and of sunspot groups through an activity cycle.
The processes generated by the $\alpha \Omega$ dynamo do not occur instantaneously. The reversal of polarity at the poles can take years. The commonly accepted mechanism for the reversal is the migration of the trailing polarity of bipolar regions towards the poles as tentatively indicated in Fig. 1.3 by the black curves. These elements of opposite polarity cancel the existing polarity and sum up for the opposite one.

Wang et al. (2002) and Baumann et al. (2004) have modelled this effect by simulations of surface magnetic flux transport. The simulations include the appearance of bipolar fields during the time of sunspot activity; diffusion of magnetic field - the magnetic field breaks up into smaller components -; differential rotation; and meridional poleward circulation - longitudinal flows directed towards the respective pole. The surface transport of small magnetic flux elements instead of monolithic sunspot tubes appears reasonable in view of the dynamic disconnection of sunspots at near-surface layers discussed by 
Schüssler \& Rempel (2005).

\subsection{Aims of the present study}

Since the solar magnetic field is so important for the global and atmospheric dynamics and characteristics of the Sun and the whole solar system, its study is of much interest and importance. Earth's orbital plane with respect to the Sun, the so-called ecliptic plane, is not too much inclined to the solar equatorial plane, as a consequence of the conservation of angular momentum of the pre-solar system nebula. Thus, the best accessible areas for observations are those near the solar equator, i.e., close to the areas of sunspot appearance. Those areas have indeed been the most investigated. Yet, during half of the magnetic cycle, the activity at the poles is of more importance than that at the equator.

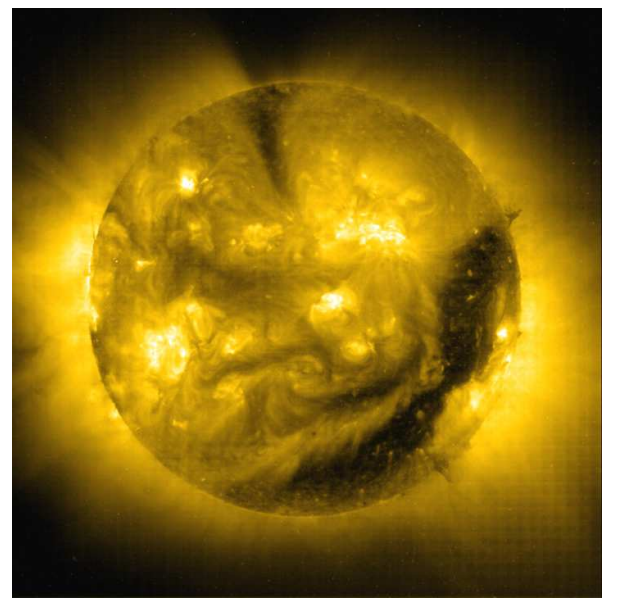

Figure 1.6: Image from SoHO/EIT.

The so-called polar coronal holes - named from their relative darkness in coronal emission lines as an effect of lower density (e.g. Figs. 1.2 and 1.6 - are largest at the time of sunspot minimum and are associated with concentrations of unipolar open field lines. These dark coronal areas (not only at the solar poles) correspond to regions of fast outflow of material (e.g. Wilhelm et al. 1998, 2000). From insitu measurements of various spacecraft (e.g. Mariner 2, Helios missions, Ulysses) the theoretically predicted solar wind (Biermann 1951) was indeed detected. It was found to occur in two kinds, the fast solar wind and the slow solar wind, according mainly to their average velocity. But, beyond this characteristic, the distinction is more complex than just different velocities. Ulysses data have shown that the fast solar wind's angular coverage, centred on the poles, is cycle dependent. During sunspot minimum the angular distribution of the fast solar wind is wider, coinciding with the polar coronal holes.

Observations of the solar poles - of the solar limb in general - from telescopes in the ecliptic plane (e.g. ground-based) have to cope with two main handicaps. First, the limb darkening, a consequence of the temperature gradient through the solar atmospheric layers together with the optical depth of observation. When observing at the disc centre - where the line of sight (LOS) is normal to the surface-, the light comes from deeper, i.e. hotter, layers than close to the limb where the LOS is more tangential. This creates a centre to limb variation of the intensity. Second, the geometrical projection effect when imaging a distant spherical surface onto a plane detector.

Nonetheless, information from very high solar latitudes is needed to analyse the behaviour of the magnetic field at the poles and its influence on the magnetic cycle, the structures appearing there and the solar wind. The Ulysses spacecraft, especially designed to study the solar wind, has been the first one to observe the Sun in an out-of-ecliptic orbit. In the near future new observational spacecraft will orbit the Sun out of the ecliptic, 
allowing to measure the magnetic fields in the very polar areas. From ground, new techniques used regularly in the last years as, e.g., adaptive optics and image reconstruction, give the possibility of studying the magnetic field and features at the poles of the Sun with an unprecedented high spatial resolution. This has allowed to partly overcome the difficulties of observing the poles.

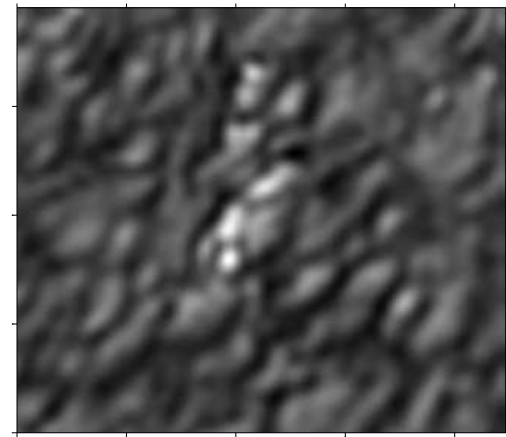

Figure 1.7: PFe example at $\mu=0.44$. Tickmark distances correspond to $2^{\prime \prime}$. Image from August 2005 campaign.
The topic of the present thesis work is to study the magnetic activity at the poles of the Sun, focusing on polar faculae (PFe; e.g. Fig. 1.7), by means of high-spatial and highspectral resolution observations. Polar faculae are conspicuously bright magnetic features of small spatial scale, with sizes of the order of $1^{\prime \prime}$ (corresponding on average to 725 $\mathrm{km}$ on the solar surface), that appear near the solar poles. They are seen in photospheric and chromospheric layers and in wavelengths from radio (Makarov et al. 1991; Riehokainen et al. 1998) to ultraviolet (Riehokainen et al. 2001). Homann et al. (1997), Okunev (2004) and Okunev \& Kneen

(2004) found their field strength to be in the kilo-Gauss range, with short timescale evolution of 1 minute, whereas remaining as an identifiable PF entity for more than 1 hour. Since they are polar magnetic features, PFe follow the activity cycle of the poles, i.e., they exhibit maximum occurrence during sunspot minimum (Makarov \& Makarova 1996). Also at that time, PFe can be found down to latitudes of $|\psi|=60^{\circ}$ while at sunspot maximum their regions of appearance vanish (Waldmeier 1955, 1962; Sheeley 1964, 1991; Makarov \& Sivaraman 1989; Makarov et al. 2003a.b). Okunev (2004) and Okunev \& Kneen (2005) performed model calculations of PFe to understand their structure. Yet still, their role with respect to the global magnetic field is not fully comprehended.

Beyond the role of PFe as contributors to the polar magnetic flux and to the magnetic activity cycle, they also qualify as possible sources of the solar wind.

Magnetic funnels, constructed from extrapolation of photospheric magnetic fields to the corona, have been suggested as origin for the solar wind by Tu et al. (2005), on the basis of magnetic measurements with MDI (Michelson Doppler Imager) and intensity and velocity measurements with SUMER (Solar Ultraviolet Measurements of Emitted Radiation; Wilhelm et al. 1995), both instruments on-board the SOHO (SOlar and Heliospheric Observatory) spacecraft. In the same manner, Wiegelmann et al. (2005) arrive to similar conclusions about equatorial coronal holes. Likewise, equatorial faculae exhibit similar properties as those of PFe. Because of the characteristics of PFe and the outflows of plasma from them observed by Okunev (2004), PFe are possible photospheric footings of the magnetic funnels. However, according to Okunev \& Kneer (2005), the numbers of PFe needed both to feed the solar wind and to obtain the magnetic flux density at Earth's distance of $3.5 \mathrm{nT}$ (Smith \& Balogh 1995) do not fit the PFe observations. Many more PFe than observed would be needed to lead to the total flux at the poles of the Sun, whereas only few PFe would be sufficient to feed the fast solar wind. 
This thesis work presents a continuation in the study of PFe and their role at global solar scale. Since previous studies (e.g. Okunev 2004) some important observational advantages have become available. One of them was noted in Sect. 1.1 with reference to Fig. 1.3 The observations for this work were performed in 2005-2007, close to the minimum of sunspot activity. Thus, the number of PFe present at the poles was higher and PFe could be found in wider regions around the poles than during sunspot maximum. Their role in the polar global magnetic field should therefore show up more clearly. In addition, several upgrades have been performed at the instruments used for the observations of the present study allowing for better spatial, spectral, and time resolution opening thus new possibilities in the analysis of the small-scale properties of PFe.

Chapter 2 contains a brief introduction to spectropolarimetry and some of its characteristics which will be used in subsequent chapters. The instruments and the telescope employed for the observations are described in Chapter 3 as well as the different campaigns when the data were taken. In Chapter 4 the data reduction processes are explained. There, also the different methods applied in the course of this study to retrieve information from the data are expounded. Chapter 5 presents the results derived from the analysis of the observations. The conclusions arising from this study are stated in Chapter 6 


\title{
2 Spectropolarimetry
}

\author{
"You think quantum physics has the answer? I mean, you know, \\ what purpose does it serve for me that time and space are exactly the same thing? \\ I mean I ask a guy what time it is, he tells me 6 miles? What the hell is that?" \\ Anything Else (2003)
}

Most of the time, the first thing told to students in an introductory course in astrophysics is that almost all we know of the universe is thanks to radiation. And that is indeed true. Since half a century, the possibility of in situ measurements is a reality, and even some particles other than photons can be measured when they arrive at Earth or at a spacecraft. These days, high-energy astrophysics is entering the multi-messenger era, where information from different messengers (e.g. neutrinos, cosmic rays, gamma rays, etc.) is correlated to study high-energy physical processes in the universe. Still, the most common, easiest and most efficient way for investigating the cosmos is by means of photons, of light.

During the last centuries, the increasing knowledge of the electromagnetic field and its properties has yielded new methods to analyse the information contained in the radiation about the material and conditions where it comes from. In the present chapter, a summary of one of those methods, spectropolarimetry, is presented (a more thoroughly description can be found in e.g. del Toro Iniesta 2003).

\subsection{Spectroscopy}

The study of the composition and properties of light started centuries ago. It is possible to follow some of the ideas through the words of the great philosophers of history, Aristotle, Descartes, Hume, ... All of them tried to explain the colours of light and its behaviour. But the first big leap in the study of the light's components was in 1672, when Newton split the white light into colours - wavelength - with a prism and then demonstrated that the prism was not the cause of the colours but the light that was formed of them. He used in his description of his experiments the word spectrum to refer to this "apparition" of colours and the term is still in use today.

Focusing on solar spectroscopy, perhaps the beginning of it was in 1802, when William Wollaston noticed some black gaps in the solar spectrum. But it was not until 1814, when Joseph von Fraunhofer designed a spectroscope and began a systematic study of these dark features, measuring the wavelengths of 574 dark lines. The dark lines both in the solar and stellar spectra are named after him: Fraunhofer lines. 


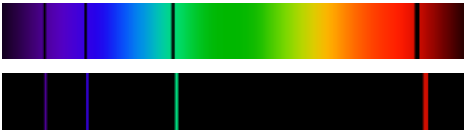

Figure 2.1: Examples of absorption (upper) and emission (lower) spectra.
The origin of the dark lines in the solar spectrum was uncovered around 1860. Gustav Kirchhoff and Robert Bunsen discovered that the spectrum of a heated gas presented characteristic bright lines. Some of these lines coincided with the dark ones observed from the Sun. It was understood later

that black lines correspond to absorption processes and bright lines to emission processes. Kirchoff and Bunsen discovered also that each chemical element produces a unique spectrum of lines. Thus, the spectrum can be used to identify the elements composing a fluid, giving birth to a new analysis tool. It was named spectroscopy, being the study of the spectrum, and was applied to astrophysics from the very beginning. Even a new element was discovered in the Sun's spectrum thanks to spectroscopy in 1868 by Pierre Janssen and Norman Lockyer. It was called Helium.

With increasing knowledge of the atomic structure, the lines were associated with the transitions of the electrons between different atomic levels. These transitions happen with only particular amounts of energy (originally quanta) absorbed or emitted,

$$
\Delta E=\frac{h c}{\lambda},
$$

where $\Delta E$ is the difference in energy between the levels of the transition, $h$ is Planck's constant - a physical quantity governing quantum physics,$- c$ the speed of light and $\lambda$ the wavelength of the transition.

These "packets" of energy are determined by the atom's electronic structure. The picture of atoms became, during the first decades of the $20^{\text {th }}$ century, more and more complex, more involved than simple orbits, as Bohr's atomic model from 1913. The more complex atomic models proved at the end capable of explaining the lines' multiplets and their response to velocity and magnetic and electric fields.

An example which is used in Chapter 5 to obtain the plasma velocities is the Doppler shift. It is based on the Doppler effect, from where the velocity of a moving light source can be obtained by means of the shift created on the original frequency of the wave due to the source's movement. It can be expressed, in the non-relativistic approximation, as

$$
\frac{\Delta \lambda}{\lambda}=\frac{v}{c},
$$

where $\Delta \lambda$ is the change in wavelength from the laboratory wavelength $\lambda, c$ is the speed of light in vacuum and $v$ the speed of the medium where the light comes from.

Since then spectroscopy has proven to be a very valuable tool for astrophysics. The constituent elements of plasmas, their abundances, ionization states, velocities, etc. can be inferred by means of spectroscopic measurements.

\subsection{Polarisation}

Around 1950, polarimetry (i.e. the measurement of the polarisation of light) arose as a new, powerful diagnosis method for astrophysics. Although the Zeeman splitting had 
already been observed in sunspots by G. Hale in 1908 and used to measure magnetic field strengths, for weak fields the splitting is not detectable (see Sect. 2.3). The measurement of polarisation - and, as a consequence, the possibility of measuring weak fields - began to be applied in solar physics by H. W. Babcock and K. O. Kiepenheuer during 1950 and following years. Nowadays it is still a tool as necessary as ever.

Polarisation is an intrinsic property of the electromagnetic field as a consequence of the plane of vibration of the field. Basically, radiation can be divided into three types: unpolarised, linearly polarised and circularly polarised. The first one refers to a field with no preferred plane of vibration of the electric vector. Linearly polarised radiation has an electromagnetic field which vibrates in just one plane. And circularly polarised radiation refers to a field whose vibration plane is rotating. An elliptically polarised field represents a more general case of the circular one, in which the amplitudes of the two components of the electric field along the main axes are not equal. A diagram of elliptical polarisation is shown in Fig. 2.2

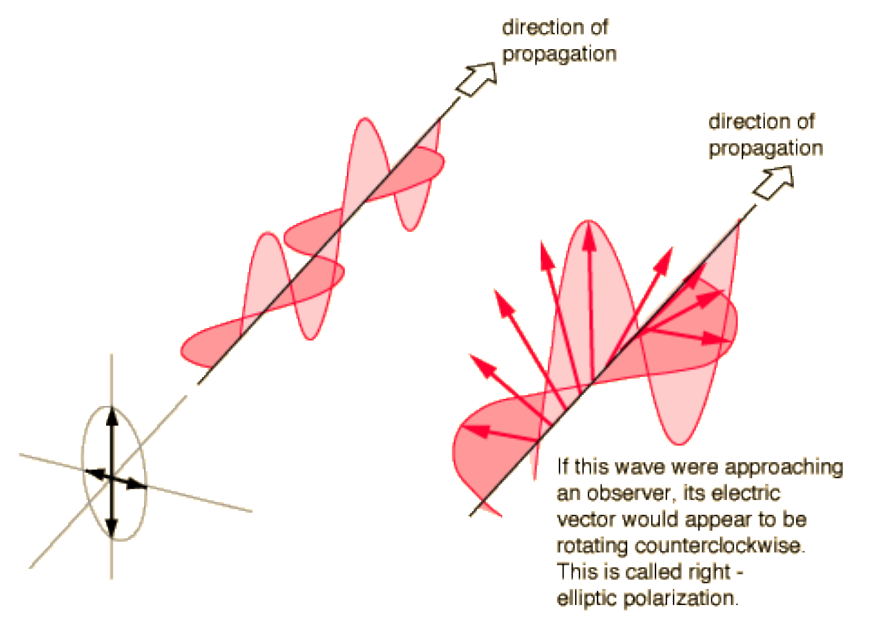

Figure 2.2: Diagram of elliptical polarisation. Image from HyperPhysics (c)C. R. Nave, 2006)

Parametrization of polarisation. In the present work, polarisation is referred to by means of the Stokes parameters, $I, Q, U$ and $V$. These parameters were first introduced by George Gabriel Stokes in 1852 as a more practical alternative to the polarisation description in terms of total intensity, degree of polarisation and shape parameters of the polarisation ellipse that was common.

The main advantage of the Stokes parameters to describe polarisation is that each parameter can be expressed in terms of additions or subtractions of measurable intensities. $I$ is the total intensity. Be $x, y, z$ a Cartesian co-ordinate system with $z$ the direction of propagation and $\vec{x}, \vec{y}, \vec{z}$ unit vectors along the corresponding axes. Angles be measured from $x$ over $y$. Then $Q$ is the intensity of the difference of the linearly polarised light at $0^{\circ}$ minus the linearly polarised light at $90^{\circ}$, whereas $U$ is the intensity of the linearly polarized light at $45^{\circ}$ minus the one at $-45^{\circ}$. Finally, $V$ is the left-circularly polarized light minus the right-circularly polarized light. By means of retarders and linear polarisers, all these intensities can be measured.

The electromagnetic field can be expressed as linear superposition of plane waves of 
different frequencies. Since the $z$ direction of the basis is the propagation direction, a monochromatic wave can be described as

$$
\begin{aligned}
& \vec{E}_{x}=E_{x} \cos (\omega t-k z) \vec{x} \\
& \vec{E}_{y}=E_{y} \cos (\omega t-k z+\phi) \vec{y} \\
& \vec{E}_{z}=0 \vec{z}
\end{aligned}
$$

with $E_{x}$ and $E_{y}$ the amplitudes and $\phi$ the phase difference between the $x$ and $y$ components. This difference in phase can be used to describe in a semi-intuitive manner the polarisation states. Linear polarisation corresponds to $\phi=0$. Then, both components are vibrating in phase and the direction of polarisation only depends on the ratio $E_{x} / E_{y}$. Circular polarisation needs two conditions: 1) $\phi= \pm \pi / 2$, and 2) $E_{x}=E_{y}$. The remaining situations correspond to elliptical polarisation.

The behaviour of polarisers and retarders can be explained in these terms also. A polariser is an optical device characterised by an acceptance axis, that is transparent to electromagnetic fields vibrating in the direction of the axis and opaque to fields vibrating in the perpendicular direction to the acceptance axis. A retarder, or wave plate, is an optical device characterised by two axes, so-called fast and slow. The effect of a retarder to an incoming electromagnetic field is to add a phase to the component parallel to the slow axis. The most common type of retarders are the quarter-wave plate, that introduces a phase of $\pi / 2$, and the half-wave plate, that adds a phase of $\pi$. With the above description of the polarisation types according to $\phi$, it is clear that such retarders can change the type and the direction of polarisation of light so that the intensities used for the measurement of the Stokes parameters can be evaluated. To illustrate this, two examples are shown in Eqs. 2.4 and 2.5 from where the Stokes parameters $U$ and $V$ can be calculated. Suppose $E_{x}=E_{y}=E, \phi=0$, and that the slow axis of the retarders are along the $y$ direction. Then,

$$
\begin{array}{rll}
\text { linear polarisation }\left(45^{\circ}\right) & \stackrel{\lambda / 2}{ } \text { linear polarisation }\left(-45^{\circ}\right) \\
\vec{E}_{x}=E \cos (\omega t-k z) \vec{x} & \Rightarrow \vec{E}_{x}=E \cos (\omega t-k z) \vec{x} \\
\vec{E}_{y}=E \cos (\omega t-k z) \vec{y} & \Rightarrow \vec{E}_{y}=-E \cos (\omega t-k z) \vec{y}
\end{array}
$$

linear polarisation $\left(45^{\circ}\right) \stackrel{\lambda / 4}{\longrightarrow}$ left circular polarisation $\stackrel{\lambda / 2}{\longrightarrow}$ right circular polarisation

$$
\begin{aligned}
& \vec{E}_{x}=E \cos (\omega t-k z) \vec{x} \Rightarrow \vec{E}_{x}=E \cos (\omega t-k z) \vec{x} \Rightarrow \vec{E}_{x}=E \cos (\omega t-k z) \vec{x} \\
& \vec{E}_{y}=E \cos (\omega t-k z) \vec{y} \Rightarrow \vec{E}_{y}=-E \sin (\omega t-k z) \vec{y} \Rightarrow \vec{E}_{y}=E \sin (\omega t-k z) \vec{y}
\end{aligned}
$$

In nature, light is never completely monochromatic, as supposed for Eq. 2.3, but it possesses a finite bandwidth in wavelength. Supposing that the bandwidth of the light is small compared to the width of a spectral line, so the phase $\phi$ can be determined, the Stokes parameters are

$$
\begin{aligned}
& I=\left\langle E_{x}^{2}+E_{y}^{2}\right\rangle \\
& Q=\left\langle E_{x}^{2}-E_{y}^{2}\right\rangle
\end{aligned}
$$




$$
\begin{aligned}
& U=2\left\langle E_{x} E_{y} \cos \phi\right\rangle \\
& V=2\left\langle E_{x} E_{y} \sin \phi\right\rangle
\end{aligned}
$$

where the averages are over the superposition of the plane waves forming the packet. Still, as pointed out by Shurcliff (1962), this expression of the Stokes parameters in terms of the electromagnetic field is complicated. Light must be assumed to be monochromatic enough so that a phase $\phi$ is definable at any time, and sufficiently polychromatic that light can be unpolarized.

The resulting polarisation of light after passing through an optical element can be determined by means of the Mueller calculus. In this method, each optical element is expressed by a Mueller matrix, a 4x4 matrix that characterises the effect of the element upon polarisation. To add the effect of various elements, the order of the matrices is inverse to the order of the elements along the optical path. The resulting polarisation state of light after $\mathrm{N}$ optical elements is

$$
\vec{S}_{o}=M_{N} \cdot M_{N-1} \cdot \ldots M_{2} \cdot M_{1} \vec{S}_{i}
$$

where $\vec{S}_{o}$ and $\vec{S}_{i}$ are the outcoming and incoming Stokes vector, respectively, $M_{1}$ is the Mueller matrix of the first optical system along the optical path and $M_{N}$ of the last one.

Instrumental Polarisation. On its way through different optical systems, the state of polarisation of the radiation undergoes modifications. In the case of the telescope used for observations for this thesis work, the instrumental polarisation, or instrumental crosstalk among the Stokes parameters, arises from retardances upon reflections at mirrors and from internal stresses of entrance and exit window of the vacuum tank (see Sect. 3.1 for description and use of the vacuum tank). Crosstalk distorts the profile of any Stokes parameter by a linear superposition of the three other parameters. The impact upon the scope of the present work is commented in Sect.4.3

\subsection{Zeeman splitting}

When in 1896 Pieter Zeeman was extending the experiments of his thesis work about interactions between light and magnetic fields, he discovered that a spectral line is split into several components in the presence of a magnetic field 2 .

As noted in Sect. 2.1 spectral lines are formed by transitions between atomic levels. Due to the several possible electronic configurations of an atom, there exist many transitions between levels occurring at the same wavelength. The presence of a magnetic field breaks the degeneracy of the atomic levels and thus, of energies of the transitions. While without magnetic field only one single spectral line appears, several can be seen with field. Furthermore, these spectral lines are polarised.

To quantify this splitting, the approximation of $L S$ coupling is adopted here. This approximation considers that the electrostatic interactions among the electrons are much more important than the spin-orbit interactions. Thus, all the electronic orbital angular

\footnotetext{
${ }^{2}$ In 1913, Johannes Stark discovered a similar behaviour, named Stark effect, in presence of an electric field instead of a magnetic field.
} 

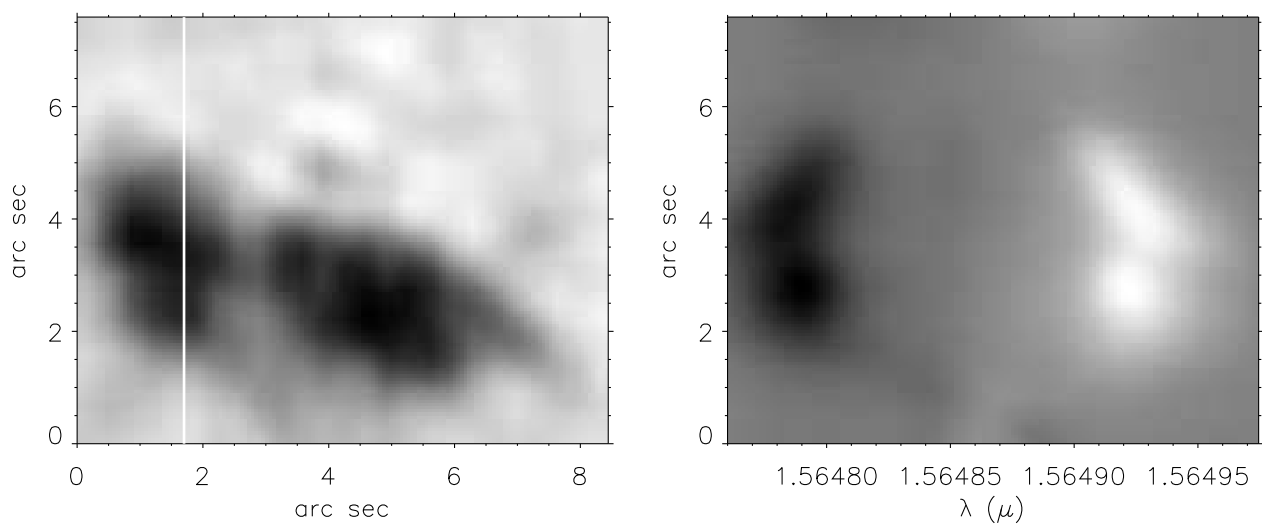

Figure 2.3: Example of Zeeman splitting. Left image shows a pore at continuum intensity at $1.56 \mu$. Right image is a Stokes $V$ spectrogram from the position corresponding to the white vertical line in the left image. The observations for this figure were taken during the May 2007 campaign, c.f. Table 3.3

momenta with quantum number $l_{i}$ couple to a total orbital angular momentum with quantum number $L$, and the analogue happens with the spin angular momenta with quantum number $s_{i}$ to form the total spin angular momentum with quantum number $S$. The total angular momentum is given by

$$
|\vec{J}|^{2}=\hbar^{2} J(J+1)
$$

with

$$
J=L+S, \quad \text { where } \quad L=\sum_{i} l_{i}, \quad S=\sum_{i} s_{i} .
$$

In this approximation, the state of an atom is defined, apart from the principal quantum numbers of the electrons, by the three above quantum numbers, $L, S$ and $J$, and the magnetic quantum number, $M_{J}$. The latter describes the projection of the total angular momentum onto a reference direction and takes the values of $-J,-J+1, \ldots J-1, J$. The state of an atom is usually written as ${ }^{(2 S+1)} L_{J}$ with $L$ represented by $S$ for $L=0, P$ for $L=1, D$ for $L=2, \ldots$

All the $(2 J+1)$ states $M_{J}$ have the same energy in the absence of magnetic field. When a magnetic field $\vec{B} \neq 0$ is present, $M_{J}$ is the projection of $J$ onto the direction of the magnetic field and the degeneracy is broken. Under such circumstances, the displacement of the line from the original position - Zeeman splitting - is

$$
\Delta \lambda_{B}=\lambda-\lambda_{0}=\frac{e}{4 \pi m_{e} c} \lambda_{0}^{2} B\left(g_{l} M_{l}-g_{u} M_{u}\right),
$$

where $e, m_{e}$, and $c$ denote the electron charge, electron mass, and speed of light, respectively, $\lambda_{0}$ the central wavelength of the transition. $g_{l}, g_{u}$ and $M_{l}$ and $M_{u}$ are the Landé factors and magnetic quantum numbers of the lower and upper states of the transition, respectively. The Landé factor is in essence a proportionality constant that relates the 


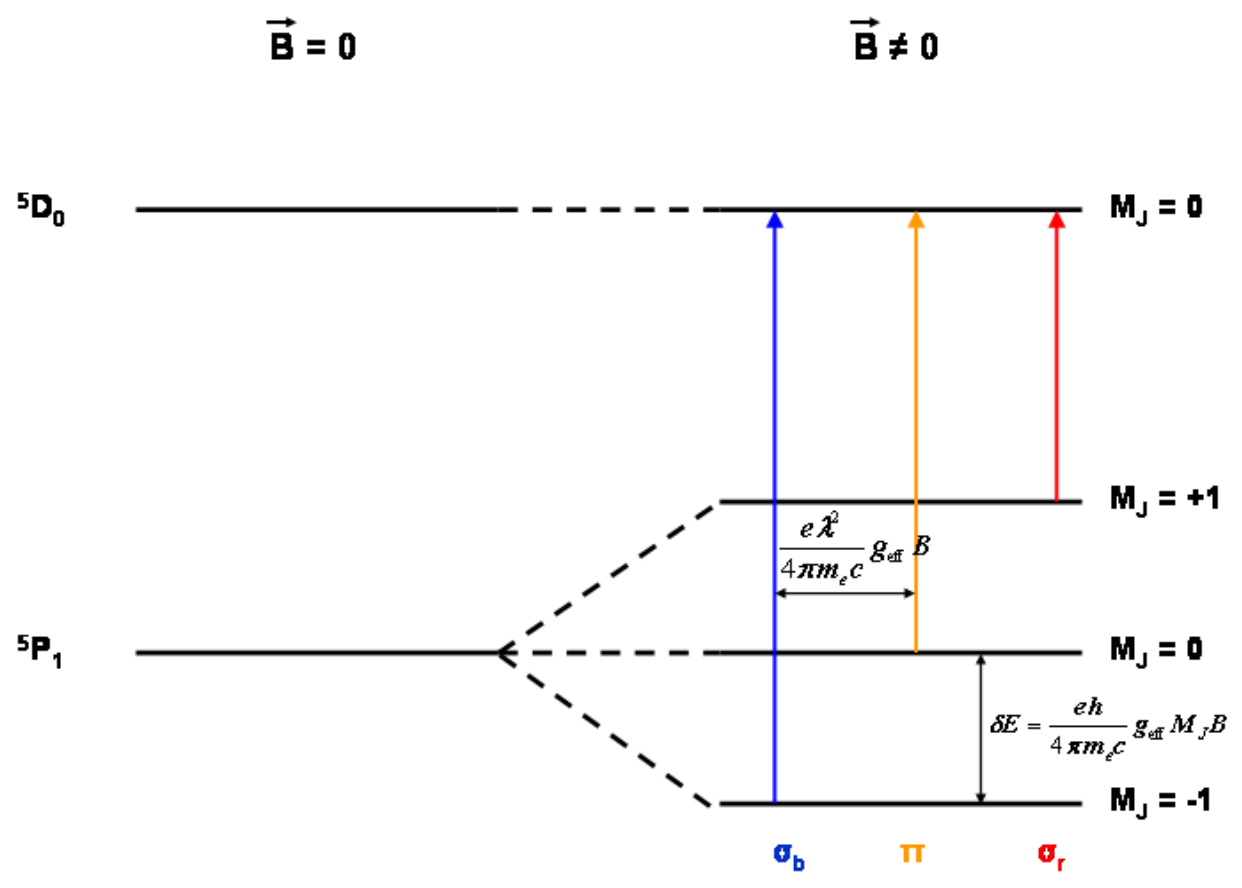

Figure 2.4: Scheme of the Fe I $6173 \AA$ A absorption without and with an external magnetic field.

magnetic moment of the electron with its angular momenta. It can be written as

$$
g=1+\frac{J(J+1)-L(L+1)+S(S+1)}{2 J(J+1)} .
$$

It is important to note that not all transitions among any $J$ and $M_{J}$ states are possible, resulting in a set of selection rules. The selection rules for the "allowed" transitions electric dipole transitions - are $\Delta J=0, \pm 1$ and $\Delta M_{J}=0, \pm 1$ (except for $J_{u}=J_{l}=0$ ). The usually so-called forbidden transitions are actually transitions that can occur, but their transition rates are much lower than the electric dipole transition.

An example with one of the lines used in this work, Fe I $6173.3 \AA$, is drafted in Fig. 2.4. This line exhibits a so-called normal Zeeman splitting, or Lorentz triplet. The names come from historical reasons. When the Zeeman effect was first discovered the quantum theory was not yet developed. Not even the spin of the electron was discovered. With the classical theory of electrodynamics, only the splitting into triplets with Landé factor $g=1$ could be explained. But in general more than three lines appear. This latter effect was called anomalous Zeeman effect as opposed to the case of a triplet, the normal Zeeman effect.

A Zeeman triplet consists of three components. One corresponding to the $\Delta M_{J}=0$ transition, or $\pi$ component, which is unshifted in wavelength. The other two correspond to the $\Delta M_{J}= \pm 1$ transitions, or $\sigma$ components, which are shifted from the central position, one to the blue and the other to the red. Depending on the angle between the LOS and the direction of the magnetic field, the relative intensities of the three components of the 
triplet are different. If the LOS is parallel to the field - so-called longitudinal Zeeman effect -, only the $\sigma$ components, which have circular polarisation of opposite sense, can be seen. With the LOS perpendicular to the magnetic field - transverse Zeeman effect -, all three components are seen, the $\pi$ component, linearly polarised parallel to the field, and the $\sigma$ components, linearly polarised perpendicular to the field. Generally, the magnetic field is oriented in-between these two extreme cases and the projection of the $\pi$ and $\sigma$ components along the LOS will be seen.

Due to the broadening of the solar lines, for weak fields - i.e., lower than the ones present in sunspots - or for small filling factors $\alpha$ - the fraction of the observational resolution element occupied by the magnetic field - the Zeeman splitting cannot be seen any more. At most, it can be noticed as a further line broadening (see Fig. 2.5 h). But the polarisation of the components together with polarimetric analyses, allows to detect the small splitting and thus, to measure weak magnetic fields that do not cause strong line splitting.

Also, since the solar lines are broad, the components of a multiplet are usually not resolved. In the case of weak field, such a multiplet can be treated as a triplet. To that end, an effective Landé factor, $g_{\text {eff }}$, is calculated from the weighted components of the multiplet. Analytically, it can be written as

$$
g_{\mathrm{eff}}=\frac{1}{2}\left(g_{u}+g_{l}\right)+\frac{1}{4}\left(g_{u}-g_{l}\right)\left[J_{u}\left(J_{u}+1\right)-J_{l}\left(J_{l}+1\right)\right] .
$$

Substituting the constants in Eq. 2.10 and using this $g_{\text {eff }}$ as a general case, the Zeeman splitting is

$$
\Delta \lambda_{B}=4.67 \times 10^{-13} g_{\text {eff }} \lambda_{0}^{2} B
$$

with $\Delta \lambda_{B}$ and $\lambda_{0}$ in $\AA$ and $B$ in Gauss. Therefore, the splitting depends linearly on the strength of the magnetic field.

The total Hamiltonian describing the energy levels of an atomic system in presence of a magnetic field is

$$
H=H_{0}+H_{M},
$$

where $H_{0}$ is the unperturbed Hamiltonian of the atom, and $H_{M}$ is the perturbation introduced by the magnetic field. The interaction of magnetic fields with atoms gives rise to three different regimes according to the strength of the field, i.e. to the ratio of $H_{M} / H_{0}$ : 1) The Zeeman regime occurs in the presence of weak fields where the magnetic field is only a perturbation to the LS coupling $\left(H_{M} \ll H_{0}\right)$. 2) For stronger fields, the LS coupling is disrupted and the spectral lines rearrange, although the perturbation introduced by the field is smaller than the original unperturbed atomic potential $\left(H_{M}<H_{0}\right)$, being then in the Paschen-Back regime. 3) For ultrastrong fields the magnetic field is no longer a perturbation and dominates over the original potential of the atom $\left(H_{M}>H_{0}\right)$, so the atom no longer exists in its normal meaning.

For most lines, fields like those found in sunspots, up to $3000 \mathrm{G}(0.3 \mathrm{~T})$, are still weak in the sense of LS coupling. Thus, the Zeeman regime is adequate for the scope of the present work.

The theoretical behaviour of the Stokes profiles can be studied from synthetic profiles derived from the radiative transfer equation for the Stokes vector (RTE; e.g. Bello González 
2006). Here, the following assumptions were made: Milne-Eddington atmosphere, static, constant magnetic field, spectral line of Fe r $6173.3 \AA$, Doppler width of $30 \mathrm{~m} \AA$ and an inclination $\gamma=40^{\circ}$ between the magnetic field and the LOS, with the latter parallel to the vertical. Thus, this situation is in-between the cases of longitudinal and transverse Zeeman effect. The variation of Stokes $I$ and $V$ with magnetic field strength ranging from $0 \mathrm{G}$ (black) to $2500 \mathrm{G}$ (red) is shown in Fig. 2.5

With regard to Stokes $I$, the three components of the triplet become only distinguishable for fields of approximately $1600 \mathrm{G}$ and stronger. For weaker fields, the only noticeable change is the increasing broadening with increasing field strength. It is commonly accepted that PFe possess a field strength below that limit, so there will be no detectable splitting in the observations of Stokes I profiles from PFe.
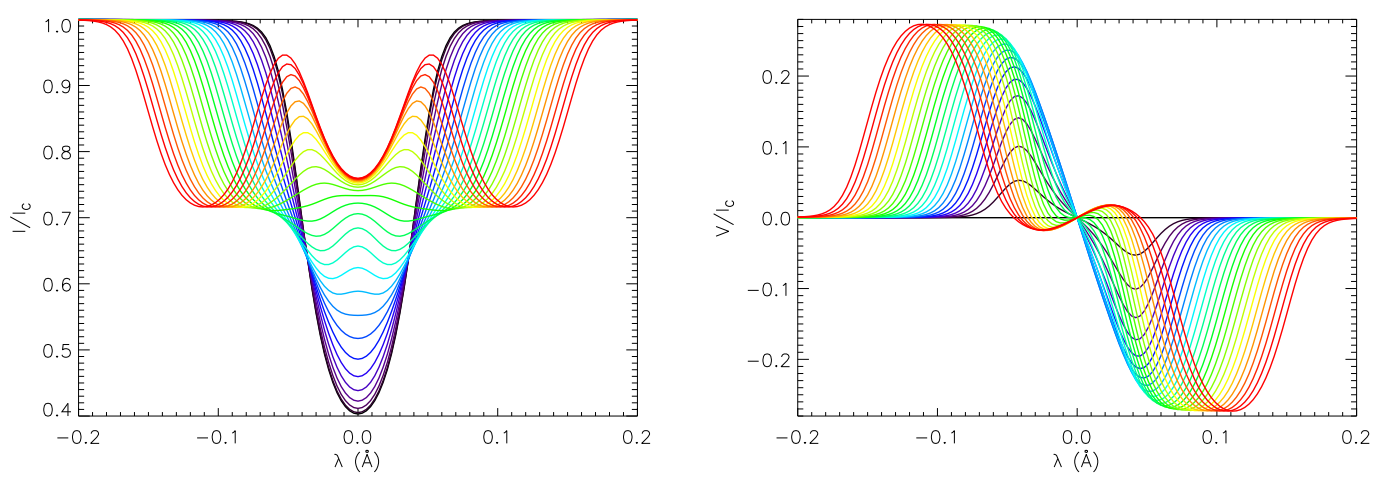

Figure 2.5: Synthetic profiles of Stokes $I$ and $V$ for Fe I $6173 \AA$, calculated under the assumptions given in the text. Color code indicates increasing magnetic field from $0 \mathrm{G}$ (black) to $2500 \mathrm{G}$ (red), in steps of $100 \mathrm{G}$.

The Stokes $V$ profiles show two antisymmetric lobes, a consequence of the opposite direction of polarisation of the two $\sigma$ components. With increasing magnetic field strength, the amplitudes of the lobes grow linearly until around $1000 \mathrm{G}$. Then, the amplitudes still become higher but with decreasing growth rate. The opposite happens with the position of the lobes. For fields weaker than $1000 \mathrm{G}$, the position of the lobes, and therefore their separation, barely changes. But for stronger fields, the separation of the extrema is the dominant effect, reaching approximately $240 \mathrm{~m} \AA$ for $2500 \mathrm{G}$. Starting from around $1300 \mathrm{G}$, small lobes of opposite sign appear close to the central wavelength. These are associated with magneto-optical effects, i.e., transformations of linear polarisation among themselves and into circular polarisation because of phase shiftings during the propagation of the light in the solar atmosphere. According to present knowledge, PFe possess field strengths in an intermediate range between the amplitude-dominated regime and the case of increasing separation. 



\title{
3 Instrumentation and Observations
}

\author{
“-Daddy's out of focus! \\ -Daddy doesn't need that, okay?" \\ Deconstructing Harry (1997)
}

This Chapter deals with the basis of the present thesis work. For the study of PFe, observations are the foundations upon which the results will be built. Thus, good observations are needed. To obtain best observations, fine instruments are required. Also, appropriate conditions of Earth's atmosphere - referred to as seeing - are desired since they determine the quality of the observations to a large extent.

In the present chapter, the telescope with which the observations were taken as well as the post-focus instruments used will be described briefly. Likewise, the difference between the two kinds of data sets obtained will be pointed out. The parameters used for the observational campaigns along with the seeing conditions during the observations will be commented in the second part of the chapter.

\subsection{Instruments}

All the observations were performed with the German Vacuum Tower Telescope (VTT) located in the Observatorio del Teide, Tenerife, at an altitude of 2400 metres. The VTT has two flat coelostat mirrors which direct the sunlight into the vertically mounted telescope. The latter consists of an off-axis, slightly aspherical primary mirror of $70 \mathrm{~cm}$ diameter and a flat folding mirror. Its focal length is $46 \mathrm{~m}$ yielding an image scale of $4.48^{\prime \prime} / \mathrm{mm}$ in the primary focus. The telescope per se - excluding the coelostat - until few meters before the focus, is contained in a vacuum tank which is evacuated to few mbar to prevent turbulent air flows near the primary mirror, heated by absorbed sunlight.

The VTT was built during mid-eighties. Presently, with its $70 \mathrm{~cm}$-aperture, it belongs to the medium-size class of solar telescopes. Larger telescopes of the $1-1.5 \mathrm{~m}$ class are in operation and under construction, while 2.5 - $4 \mathrm{~m}$ solar telescopes are being designed. A scheme of the telescope, with its main features of interest for this work marked with coloured shaded areas, is depicted in Fig. 3.1.

Very close to the entrance window of the vacuum tank, some small part of the light is deflected to feed the guiding system for the coelostat. Behind the vacuum exit window, the adaptive optics (AO) is located. After the AO the light can be directed into an optical laboratory where the "Göttingen" Fabry-Perot interferometer (FPI) is located. Alternatively - or simultaneously via a dichroic beam splitter - the light can continue to the 


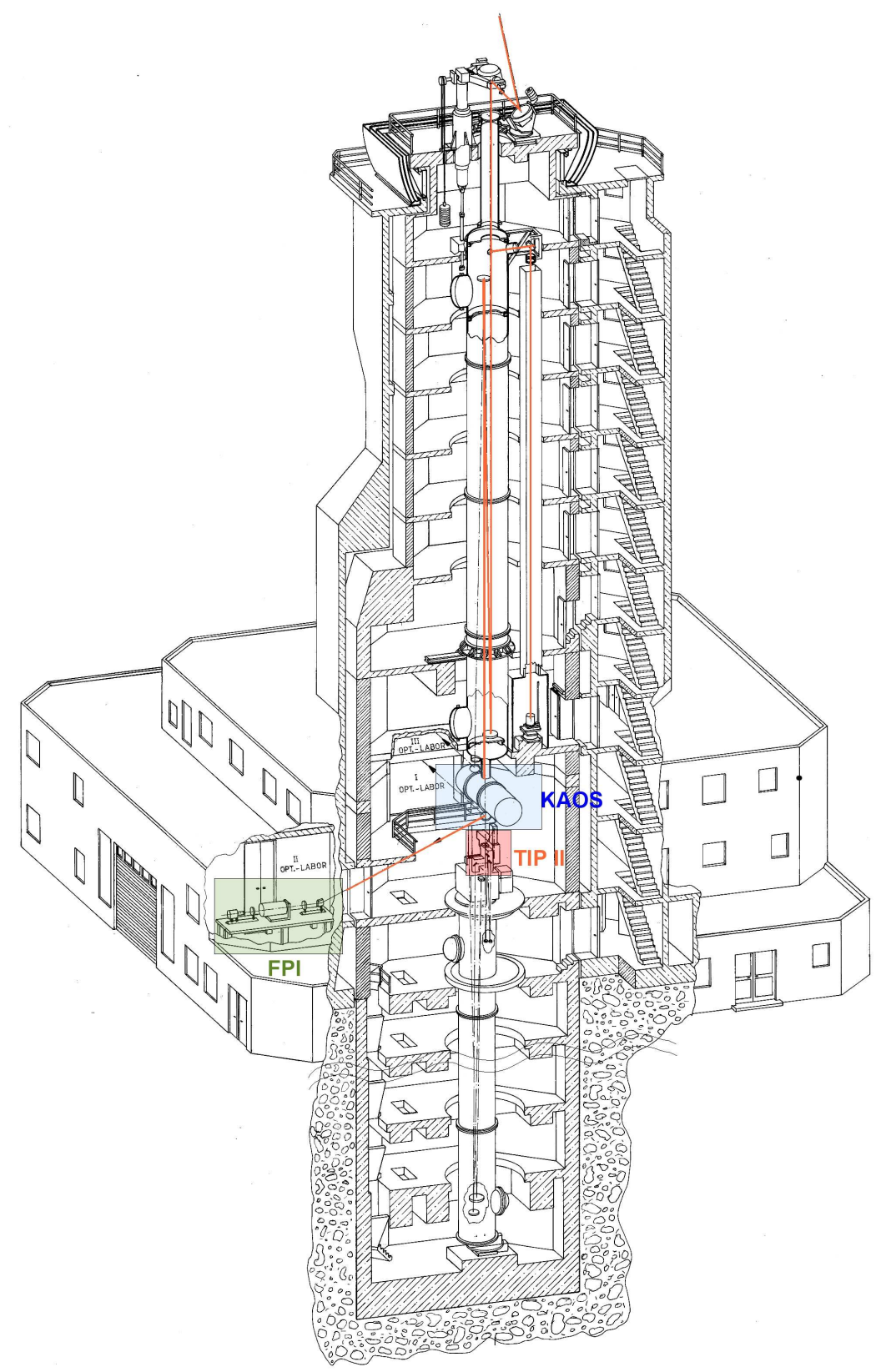

Figure 3.1: Scheme of the VTT and building. In the scheme the path of the light depicted as an orange line and the three parts described in the following subsections are marked as blue, green and red shaded areas.

vertically mounted slit spectrograph. At the exit of this spectrograph, the Tenerife infrared polarimeter II (TIP II) is seated.

During the last years, new post-focus instruments have been added at the VTT. Also, upgradings of existing instruments have been performed. An AO system has come into regular operation, allowing high-quality observations with high spatial resolution. Among the upgraded instruments are the FPI and TIP II, which are used for this study. Both of them will be described below. The upgradings will be completed for the new $1.5 \mathrm{~m}$ solar telescope GREGOR at the Observatorio del Teide. The construction of GREGOR is close 
to be completed, and will likely come into operation in late 2008 . With the new postfocus equipment, it will allow unprecedented spatial resolution. For the present work, high spatial resolution is of much importance, since PFe are small-scale structures whose study requires detailed information.

Before the description of the three elements marked in Fig. 3.1, a short note on the properties of FPI and TIP II is appropriate.

The FPI is a two-dimensional (2D) spectrometer. Fabry-Perot etalons are the heart of this kind of instrument. With it, a 2D field of view (FOV) is observed through a very narrow wavelength band $(20-50 \mathrm{~m} \AA)$. Scanning across a spectral range, e.g. through a spectral line, is performed by changing the etalon spacings by means of modulating the voltage applied to Piezo crystals which control the spacing.

TIP, which consists of a polarimetric analyser and of a CCD detector, makes use of the grating spectrograph of the VTT. This kind of instrument disperses in wavelength the incoming light which enters the spectrograph through a slit. So one observes simultaneously a certain wavelength range from all image points along the slit. Therefore, a $2 \mathrm{D}$ FOV is obtained by scanning the solar image perpendicularly to the slit direction.

Examples of spectrometric data from FPI and spectrographic TIP observations are shown in Figs. 3.2 and 3.3

Both observational methods are complementary and have their advantages and disadvantages. Slit spectrographs can have a high spectral resolution and can cover a large spectral range simultaneously. Yet the scanning of a 2D FOV is time-consuming and the spatial resolution suffers from variable seeing conditions. On the other hand, 2D spectrometers based on FPI etalons can cover a large FOV at once, which furthermore admits the application of image reconstruction methods. However, 2D spectrometers need spectral scanning and the spectral resolution is usually lower than that of slit spectrographs, since only few wavelength positions are commonly covered by observations with FPIs. Also, the sequential scanning may hamper the study of fast processes.

\subsubsection{Kiephenheuer Adaptive Optics System}

The Kiepenheuer Adaptive Optics System (KAOS, von der Lühe et al.2003), may be considered as the most important upgrade for the VTT in the last years. It allows, under good seeing conditions, to achieve an angular resolution close to the diffraction limit, which is $0.177^{\prime \prime}$ for a $70 \mathrm{~cm}$ entrance pupil and a wavelength of $6000 \AA$.

Adaptive optics systems operate in real time, during the observations. They are designed to correct the wavefront aberrations caused by turbulence in the Earth's atmosphere due to temperature gradients and winds. This turbulence perturbs the optical path of the incoming light. For small apertures, like the pupil of the human eye, the atmospheric turbulence results in amplitude fluctuations of the waves (twinkle of stars). For larger apertures, the effects are image motion and blurring.

A short description of the functioning of KAOS may suffice here (for further information see Sailer 2006). The main constituents of KAOS are a Shack-Hartmann wavefront sensor, a tip-tilt mirror and a deformable mirror. To perform all the calculations for the wavefront corrections as quickly as possible, a fast computer with powerful software is also needed. 

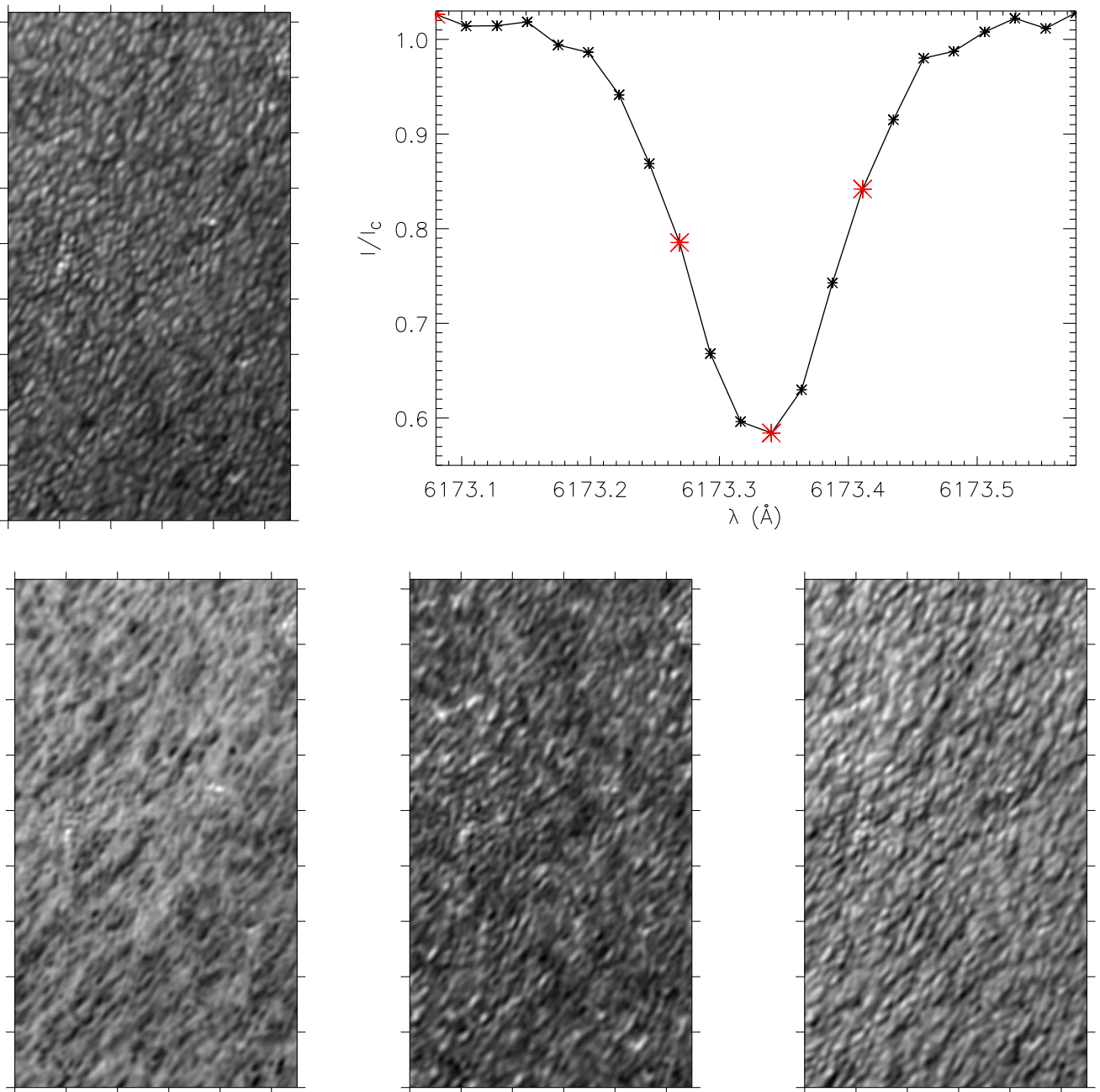

Figure 3.2: Example of images at different wavelength positions of the Fe I $6173 \AA$ Aline, obtained with the FPI spectrometer. The upper left image is the continuum image. The upper right plot represents the Stokes I profile averaged over the whole FOV and depicts as asterisks the positions along the $6173 \AA$ spectral line where observations where made. Red asterisks correspond to the presented images. The lower row shows images at wavelength positions $-70 \mathrm{~m} \AA$ off line minimum (left), line minimum (centre) and $+70 \mathrm{~m} \AA$ off line minimum (right). Tickmark intervals correspond to $5^{\prime \prime}$. The observations for this figure stem from the August 2005 campaign, cf. Table 3.2

A small amount of the incoming light is directed to the wavefront sensor located in an image of the entrance pupil. There a lenslet array of 36 small lenses produces 36 images of a small subfield in the FOV, the so-called lockpoint of the AO. When the incoming wavefront is unperturbed, the images are identical. But with deformed wavefronts the images are shifted from their zero position given by a reference image. These shifts are used by means of a correlation algorithm to calculate the needed correction for the wavefront. For this algorithm to work properly, a high contrast object is desirable in the lockpoint. At the disc centre, mid seeing conditions transmit the granulation pattern with sufficiently high contrast. With worse conditions or closer to the limb, a conspicuous feature - e.g. sunspot, pore, $\mathrm{PF}$ - is necessary.

The information about the corrections is then translated into electric voltages to be applied 

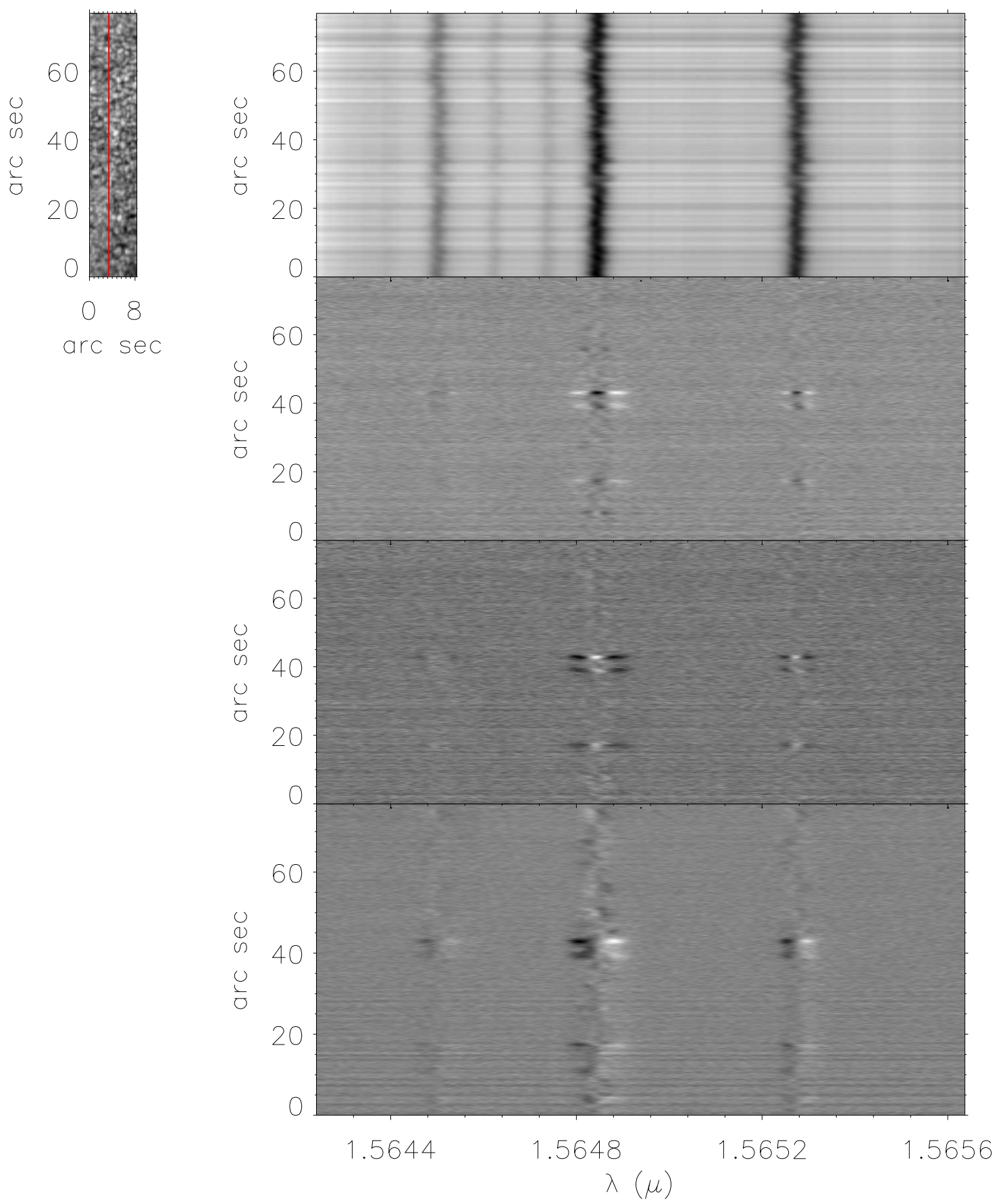

Figure 3.3: Example of spectrograph data. The left image is a composition of continuum data from 25 different scanning positions of the solar image. The right column contains the Stokes I (upper row), $Q, U$ and $V$ (lower) data corresponding to the red vertical line in the continuum image. The observations for this figure were obtained during the May 2007 campaign, cf. Table 3.3

to the tip-tilt mirror and the deformable mirror. The tip-tilt mirror is a flat mirror which is turned about two axes to hold the image as still as possible. The deformable mirror is of bimorphous material. It has 35 actuators that change the shape of the mirror's surface to correct for the wavefront deformation.

Under adequate seeing conditions, the resulting image from KAOS appears with much 
improved quality.

Yet, AO systems do not work very well at image positions in the FOV far away from the lockpoint. The reason for this spatial dependence of the AO correction is that the wavefront deformations are only approximately constant within small solid angles, the so-called isoplanatic patches. Since the wavefront correction is calculated for the lockpoint, the farther the image position is from the lockpoint, the less accurate the correction becomes. This effect is especially important for large FOVs, like the ones observed with the FPI spectrometer of $77^{\prime \prime} \times 58^{\prime \prime}$.

This problem will likely be solved with the advent of the new generation of adaptive optics systems, the multi-conjugate adaptive optics (MCAO), which is being developed for the GREGOR telescope. It corrects also for the effects of turbulence at high atmospheric layers, which are responsible for the reduced angular size of the isoplanatic patches. This correction is made by means of a further deformable mirror, positioned at the image plane of these layers.

\subsection{2 “Göttingen” Fabry-Perot Interferometer}

The 2D Fabry-Perot spectrometer was designed and constructed in its first version by the Universitäts-Sternwarte Göttingen in the early 1990s (Bendlin et al. 1992; Bendlin 1993; Bendlin \& Volkmer 1995). Since then, it has been upgraded several times (see e.g. Koschinsky et al. 2001; Puschmann et al. 2006). The last improvement, which included full Stokes polarimetry and an increase of the spectral resolution (Bello González \& Kneen 2008), was only implemented in early 2007. The observations for this thesis were taken earlier. Thus this work could not benefit from the last upgrade.

Figure 3.4 shows a basic scheme of the FPI. It is specifically designed and built to allow 2D spectropolarimetric observations with high spatial resolution applying speckle methods. The FPI possesses essentially two optical trains. A beam splitter, located close to a field stop in a focal plane, directs $5 \%$ of the light into the broadband train and $95 \%$ into the narrow-band train.

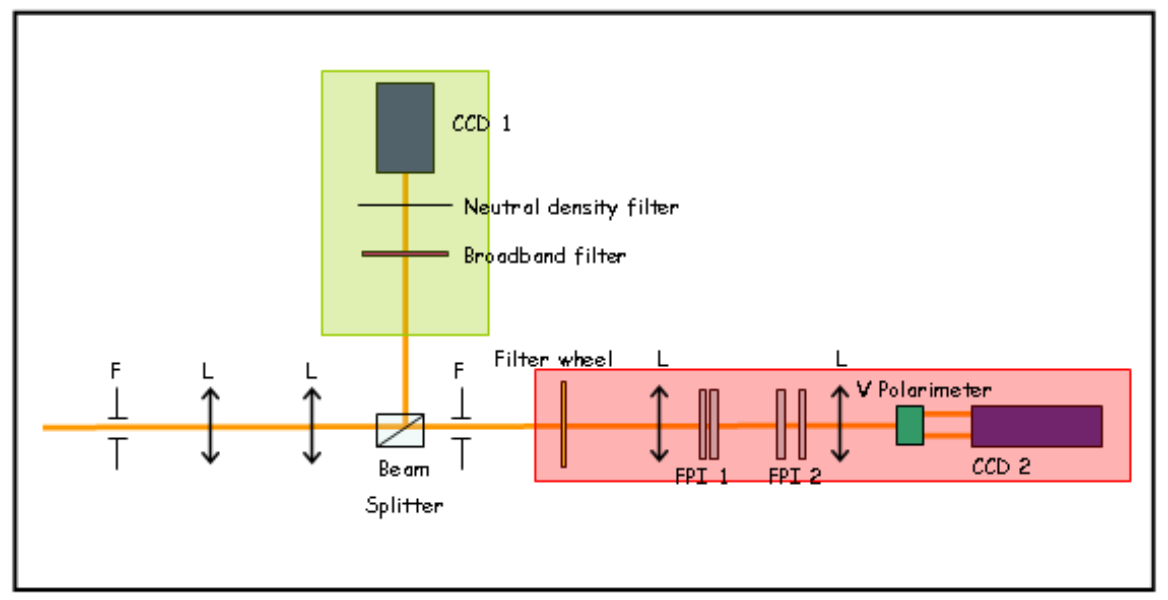

Figure 3.4: Scheme of the FPI. The greenish shaded area corresponds to the broadband and the reddish area to the narrow-band train, respectively. L: lense. F: field-stop in focal plane. 
The narrow-band train is the core of the instrument. It consists basically - apart from lenses to transfer the focal planes, interference pre-filter and a laser and photomultiplier for calibration - of a narrow-band interference filter, with a typical FWHM of $10 \AA$, and two Fabry-Perot etalons. This part of the instrument allows to make spectropolarimetry. The two Fabry-Perot etalons, with spacings of $d=1.1 \mathrm{~mm}$ and $d=0.125 \mathrm{~mm}$ at the time of the observations, form the heart of the spectrometer. For this reason, using pars pro toto, the whole spectrometer is often termed "Göttingen" FPI. Spectrometric scans are performed by changing the spacings $d$ of the etalons, thus changing the wavelength positions of the transmission peaks of the etalons. This way one can select specific wavelengths across a spectral line.

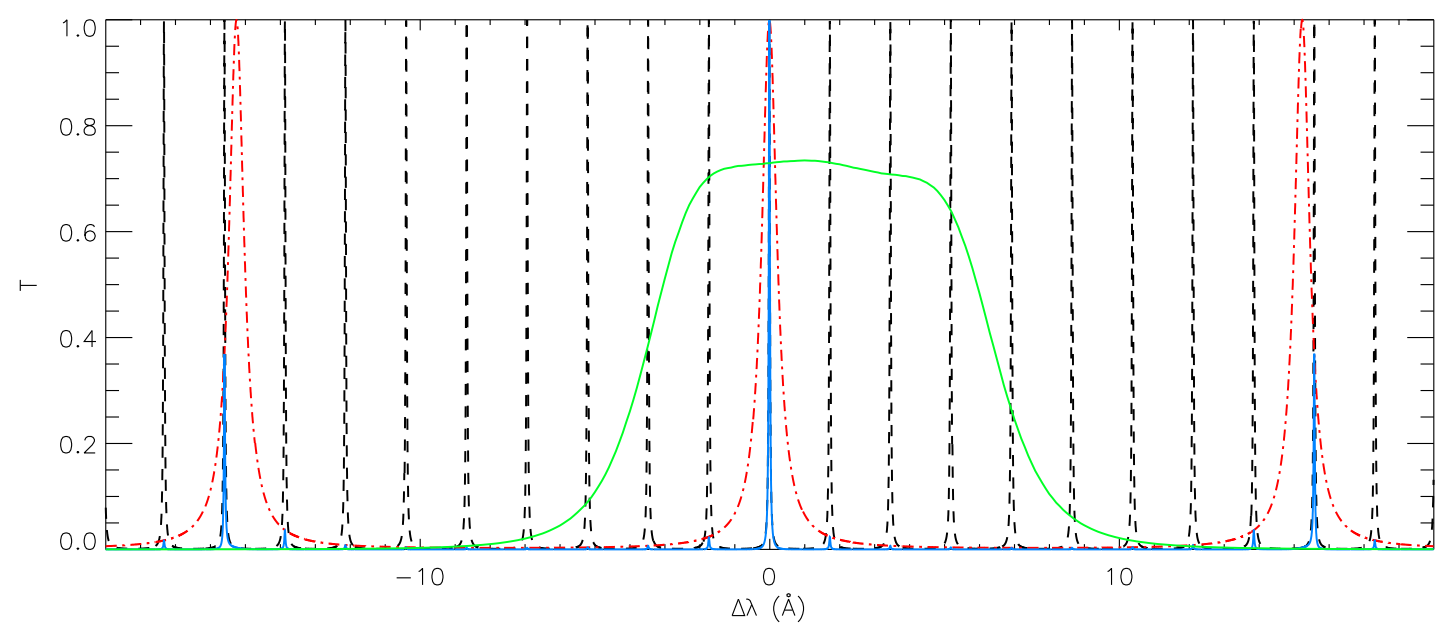

Figure 3.5: Transmissions from the interference filter (solid green line), the broadband etalon (dashdotted red line), the narrow-band etalon (dashed line), and the combination of the two latter (solid blue line) around the central wavelength.

Figure 3.5 shows how the two etalons and the narrow-band prefilter act together to select a narrow transmission peak of the $1.1 \mathrm{~mm}$ etalon.

One FPI produces a channel spectrum with a separation of adjacent orders, or free spectral range $(F S R)$ of

$$
F S R=\frac{\lambda^{2}}{2 n d \cos \Theta}
$$

where $n$ is the refractive index of air (a value $n=1$ is used throughout the present work), $d$ the spacing of the plates, and $\Theta$ the angle of incidence of the light onto the reflecting surfaces of the etalon - for the moment, a value $\Theta=0^{\circ}$ is assumed. At a wavelength $\lambda=6173 \AA$ - one of the solar spectral lines used in this work -, the $1.1 \mathrm{~mm}$ etalon has a FSR of $1.73 \AA$ and the $0.125 \mathrm{~mm}$ etalon a FSR of $15.24 \AA$. The purpose then of the broadband etalon is to suppress the adjacent orders of the small-band etalon. As can be seen from Fig. 3.5, the composite curve of both etalons shows only a very small influence from the neighbouring transmissions, less than 5\%. The additional interference filter, whose transmission curve is also seen in Fig. 3.5 as a green curve, selects one order from the broad etalon impeding the influence from the other orders.

Finally, a Stokes $V$ polarimeter was placed just in front of the CCD detector. This polarimeter, by means of a $\lambda / 4$ retarder plate and a pair of calcites, splits the incoming 
light in its two circularly polarised components, $\frac{1}{2}(I+V)$ and $\frac{1}{2}(I-V)$. Each of these components or channels, illuminates a different half of the detector and can later be treated separately or combined to obtain the Stokes parameters $I$ and $V$.

The mounting of the instrument is also important for the final result of the observations. The FPI etalons are mounted in the collimated - i.e. parallel - beam close to an image of the telescope pupil. Unlike having them in a telecentric mounting - which causes the orange peel pattern due to small imperfections of the flatness of the etalon surfaces -, the collimated mounting results in a blueshift. This terminology refers to a shift of the maximum of wavelength transmission across the FOV. The interference condition of FPIs for maximum transmission is

$$
m \lambda=2 n d \cos \Theta,
$$

with $m$ being the order. For small angles of incidence $\Theta$, the same order $m$ and $n=1$, one arrives at the blueshift

$$
\Delta \lambda=-\lambda(\cos \Theta-1) \approx-\frac{\lambda}{2} \Theta^{2} .
$$

The blueshift can be corrected as will be pointed out in Sect. 4.1. For more information about advantages and disadvantages of the two mountings, see Kneer \& Hirzbergen (2001).

The broadband part of the instrument is composed of a broadband filter, with a FWHM of typically 50 - $100 \AA$, a neutral density filter (NF) and a CCD detector (CCD1). The NF prevents the saturation of the CCD by an excess of photons. Here in the broadband train, a high number of short-exposure frames - more than one hundred - is taken, which will allow the post-facto speckle reconstruction of the data. Speckle methods are techniques of image reconstruction which improve the spatial resolution of the images taken during the observations. These techniques will be explained in more detail in Sect. 4.2 .

The upgrades of the FPI described in Puschmann et al. (2006), have increased its efficiency by a factor of approximately 60 .

The new CCDs, from LaVision GmbH, Göttingen, possess substantially more pixels and higher quantum efficiency, and allow much higher frame rates than the former CCDs. The new size of the FOV is $73^{\prime \prime} \times 55^{\prime \prime}$ for the broadband images and approximately $33^{\prime \prime} \times 54^{\prime \prime}$ for each narrow-band channel when using the Stokes $V$ polarimeter, with a pixel size corresponding to $0.112^{\prime \prime} \times 0.112^{\prime \prime}$ for both cameras. Thanks to these new CCDs also the exposure times could be reduced from $30 \mathrm{~ms}$ to 5-10 ms. This is important for the application of speckle methods which require that the atmospheric seeing conditions be frozen, i.e. constant, during exposure.

A further upgrade concerns the software specially developed for the spectrometer control and data acquisition. It is now possible to scan consecutively several spectral regions. For this purpose, various narrow-band interference filters for the corresponding wavelength range can be mounted on a filter slider and be moved into, and out of, the light beam.

The structure of an observation of a spectral scan consists of short-exposure narrowband frames at some 20-30 wavelength positions across a spectral line, with a sampling in wavelength appropiate for the FWHM of the spectrometer's transmission curve. Most 
times, at each wavelength position, 5-20 frames are taken. This is important for image reconstruction (see Sect.4.2). Also important for the image reconstruction, short-exposure frames of the same FOV are taken with the broadband CCD1 strictly simultaneously with the narrow-band images. In addition to these data, dark frames are recorded, as well as flat fields and frames from a continuum light source, which will allow to perform the data reduction (see Sect. 4.1).

In Fig. 3.6, an example of a spectral line profile, from Fe I $6173.3 \AA$, obtained in April 2006 is shown. In addition, the FPI transmission as well as the line profile from the Fourier Transfrom Spectrometer (FTS) Atlas (Brault \& Neckel, quoted by Neckel 1999) are shown. The FTS possesses a very high spectral resolution and the line profiles from it may be considered as free of any instrumental broadening, compared to the width of the solar spectral lines.

As can be seen in Fig. 3.6 the line depression of the observed data is much lower than

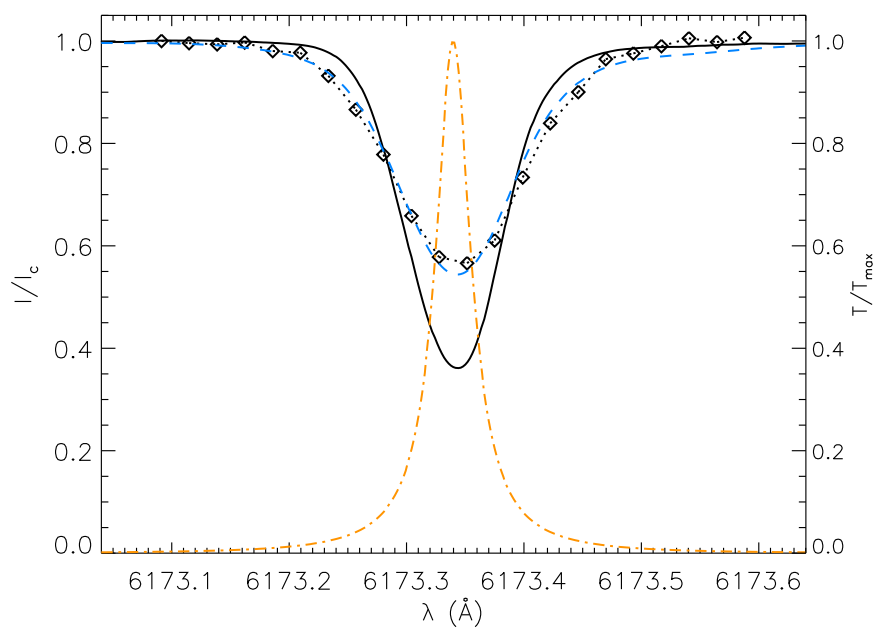

Figure 3.6: Intensity profiles from the FTS atlas (solid line), the observed data (rhombuses connected by dotted line) and the convolution of the FTS data with the Airy function from our instrument (blue dashed line). The Airy function from the instrument is drawn as an orange dash-dotted line with the scaling at the right axis.

the one from the FTS atlas. To simulate the effect of the FPI we have convolved the FTS data with the transmission curve of the combined two etalons. The resulting line profile has come close to the observed profile.

\subsubsection{Tenerife Infrared Polarimeter II}

TIP II is the new version of the original TIP (Collados 1999; Martínez Pillet et al. 1999; Collados et al. 2007). It possesses a better and bigger infrared-sensitive CCD chip than TIP, with 1024x1024 pixels. It is operating since May 2005 and has already achieved its optimum performance.

TIP II is an infrared polarimeter which makes use of the VTT's spectrograph. It measures the four Stokes parameters by means of a couple of ferroelectric liquid crystals (FLCs) and is able to achieve a polarimetric sensitivity of few times $10^{-4} I_{c}$, where $I_{c}$ is the continuum intensity. The FLCs are retarders which, according to an applied voltage, can change the 
orientation of their slow axes between two positions. Seen along the optical path, the first FLC possesses a (nominal) retardance of $\lambda / 2$ and the second FLC of $\lambda / 4$. The change in orientation amounts to (nominally) $45^{\circ}$ for each of the FLCs. The signal sent to the $\lambda / 2$ retarder has twice the frequency of the $\lambda / 4$. Therefore, a complete cycle of the polarimeter is composed of four different states. In each of the states, combinations of the four Stokes parameters are measured, so that at the end $I, Q, U$ and $V$ can be retrieved by combining the four states.

In terms of the modulation matrix of the polarimeter, $\boldsymbol{M}$, one has

$$
I=M \cdot S
$$

where $\boldsymbol{I}$ is the measured vector of four states and $S$ is the Stokes vector of the incoming light. To obtain $S$ from the observations, the inverse of the modulation matrix is applied,

$$
\boldsymbol{S}=\boldsymbol{M}^{-1} \cdot \boldsymbol{I}=\boldsymbol{D} \cdot \boldsymbol{I},
$$

with $\boldsymbol{D}$ being the demodulation matrix.

The temporal modulation of the polarised light by means of the FLCs is accompanied by a spatial modulation. The latter is achieved with a polarising beamsplitter, and the two outcoming beams reach different parts of the same detector. The combination of both methods to take observations on a two-dimensional detector allows to minimise the effects of crosstalk, both of gain-table uncertainties, which come from using only a spatial modulation, and of seeing-induced crosstalk arising from the temporal modulation.

For a demonstration of the low $I \rightarrow Q, U, V$ crosstalk thanks to the double modulation, consider a simple case where only Stokes $I$ and $V$ are measured. The intensities observed at the detector are

$$
\begin{aligned}
& I^{+}\left(t_{1}\right)=(I+V) / 2 \\
& I^{+}\left(t_{2}\right)=(I+\delta I-V-\delta V) / 2 \\
& I^{-}\left(t_{1}\right)=\left(1+\delta_{g}\right)(I-V) / 2 \\
& I^{-}\left(t_{2}\right)=\left(1+\delta_{g}\right)(I+\delta I+V+\delta V) / 2,
\end{aligned}
$$

where + and - refer to the two beams (spatial modulation) and $t_{1}$ and $t_{2}$ to the two states of the FLCs (temporal modulation). $\delta I$ and $\delta V$ account for the seeing-induced crosstalk and $\delta_{g}$ represents the gain-table uncertainties.

Combining the four equations yields

$$
\begin{aligned}
I_{\text {meas }}= & \frac{1}{2}\left[I^{+}\left(t_{1}\right)+I^{+}\left(t_{2}\right)+I^{-}\left(t_{1}\right)+I^{-}\left(t_{2}\right)\right] \\
& =\left(1+\delta_{g} / 2\right)(I+\delta I / 2)+\delta V \delta_{g} / 4 \\
V_{\text {meas }}= & \frac{1}{2}\left[I^{+}\left(t_{1}\right)-I^{+}\left(t_{2}\right)-\left(I^{-}\left(t_{1}\right)-I^{-}\left(t_{2}\right)\right)\right] \\
& =\left(1+\delta_{g} / 2\right)(V+\delta V / 2) V+\delta I \delta_{g} / 4
\end{aligned}
$$

The last term in Eq. 3.11 corresponds to the crosstalk $I \rightarrow V$. In most cases, $\delta I \delta_{g} / 4 I$ is of the order of $10^{-3}$ and smaller, thus it can be neglected. 


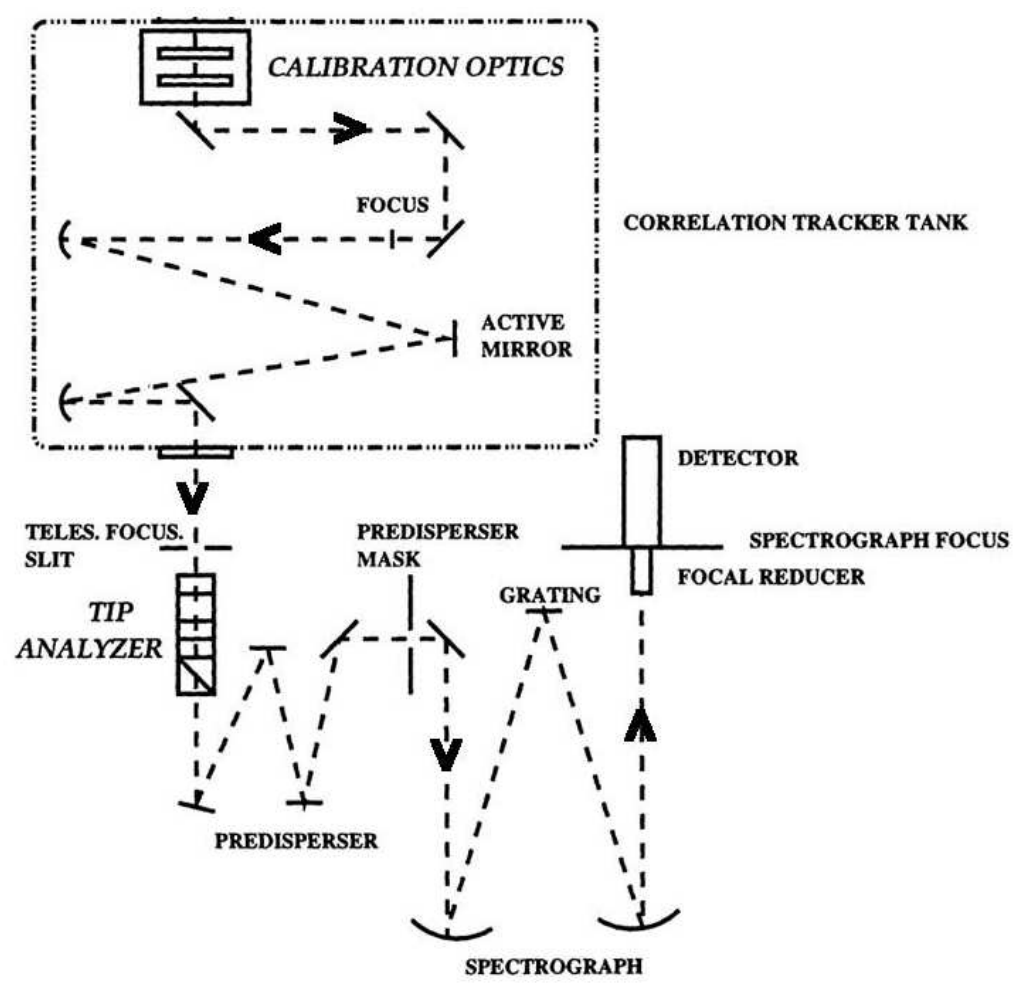

Figure 3.7: Scheme of TIP and spectrograph organisation (from Collados 1999).

After passing through the polarimetric analyser system, the light enters the spectrograph, mounted vertically and located mainly below the ground level of the VTT. The dispersed light is detected by the TIP II CCD. The latter is mounted inside a cryostat which keeps it at a temperature below $80 \mathrm{~K}$ to suppress the thermal excitations in the CCD chip and to avoid infrared stray light from the surroundings. Inside the cryostat there are also three filters for selection of the wavelength range. For the present work, the one for $1.56 \mu$ was used, which has a transmission of $90 \%$ at that wavelength.

A scheme of the light path from the exit of the telescope's vacuum tank through TIP and the spectrograph to the detector is shown in Fig. 3.7. The calibration optics is also depicted there. This system consisting of a linear polariser and a retarder serves two purposes: 1) to calibrate the FLCs, and 2) to measure the instrumental polarisation induced by all the optical elements from the exit of the vacuum tank, through KAOS, to the detector.

For the spectrograph, there is a set of slits with different widths from $40 \mu \mathrm{m}$ to $120 \mu \mathrm{m}$. The width of the chosen slit has various effects on the observations. On the one hand the wider the slit, the higher the number of photons gathered. This implies that observations can be made faster and with better signal to noise $(\mathrm{S} / \mathrm{N})$ ratio. On the other hand, a narrower slit allows better spectral resolution and gathers light from a smaller area of the Sun, i.e. gives higher spatial resolution than a wider slit.

To increase the S/N ratio, TIP II offers the possibility of taking various cycles or accumulations, $N_{A}$. This means that the four states are measured $N_{A}$ times and the corresponding counts are added. Supposing the noise of each measured state - e.g. due to 
photon noise - to be approximately the same, $\sigma$, the noise of each Stokes Parameter, $\sigma_{i}^{\prime}$ is

$$
\sigma_{i}^{\prime 2}=\sigma^{2} \sum_{j=1}^{4} D_{i j}^{2},
$$

just by applying error propagation to Eq. 3.5 This is the noise per modulation cycle. Now, for a number $N_{A}$ of cycles and with the efficiencies of the device, $\epsilon_{i}$, defined as (e.g. Sánchez Almeida et al. 1994)

$$
\boldsymbol{\epsilon}_{i}=\left(4 \sum_{i=1}^{4} \boldsymbol{D}_{i j}^{2}\right)^{-\frac{1}{2}}
$$

the noise can be written as

$$
\sigma_{i}^{2}=\frac{\sigma_{i}^{\prime 2}}{N_{A}}=\frac{\sigma^{2}}{N_{A}} \frac{1}{4 \epsilon_{i}^{2}} .
$$

Then, the $\mathrm{S} / \mathrm{N}$ ratio of the Stokes parameters is

$$
(S / N)_{i}=(s / n) \epsilon_{i} \sqrt{4 N_{A}}, \quad i=1,2,3,4,
$$

where $(\mathrm{s} / \mathrm{n})$ is the signal-to-noise ratio of one measurement of each of the four states.

The former instrument, TIP, was upgraded to TIP II with a bigger FOV of about 77" and better spatial resolution, with half the pixel size of TIP. The resolution in the spectral direction has also been improved to twice the original one, and a wider wavelength coverage is obtained because of the bigger CCD chip. The observable spectral range is likewise extended, going now from 0.9 to 2.5 microns.

A summary of the main characteristics of TIP and TIP II is given in Table 3.1

\begin{tabular}{|c|c|c|}
\hline & TIP I & TIP II \\
\hline \hline Spatial sampling & $0.36^{\prime \prime} / p x$ & $0.18^{\prime \prime} / p x$ \\
\hline Spatial coverage & $36^{\prime \prime}$ & $77^{\prime \prime}$ \\
\hline Spectral sampling & & \\
$1.08 \mu \mathrm{m}$ & $29.6 \mathrm{~m} \AA / \mathrm{px}$ & $11.6 \mathrm{~m} \AA / \mathrm{px}$ \\
$1.56 \mu \mathrm{m}$ & $29.4 \mathrm{~m} \AA / \mathrm{px}$ & $15.6 \mathrm{~m} \AA / \mathrm{px}$ \\
\hline Spectral coverage & & \\
$1.08 \mu \mathrm{m}$ & $7.6 \AA$ & $11.8 \AA$ \\
$1.56 \mu \mathrm{m}$ & $7.5 \AA$ & $15.9 \AA$ \\
\hline
\end{tabular}

Table 3.1: Summary of the main characteristics of TIP I and TIP II (after Collados et al. 2007)

\subsection{Campaigns}

The observational campaigns are split into two groups according to the two instruments used: the ones in which the FPI was used and the ones with TIP II.

During the campaigns, with both post-focus instruments, $\mathrm{PFe}$ were identified visually in 
live images from a video camera. Since PFe were needed as lockpoint for KAOS (cf. Sect. 3.1.1, a systematic coverage of the polar areas of the Sun could not be performed. The intention was to observe PFe both close to the limb and at low heliocentric angles, i.e. as close to the disc centre as possible. Thus, observations were constrained by the heliographic latitude of the disc centre of the Sun, $B_{0}$. For positive $B_{0}$ the solar north pole is better observable than the south pole, and vice versa for negative $B_{0}$. Some data were also taken from faculae near the solar equator, for comparison with PFe.

The campaigns with the FPI were performed in April/May 2005, August 2005 and April 2006. The observing conditions were very variable along the campaigns. An adequate measure for the quality of the atmosphere for observations is the Fried parameter $r_{0}$ (Fried 1966). An average $r_{0} \sim 10 \mathrm{~cm}$ was estimated at the times when data were taken. During the last FPI campaign in April 2006, the sky was often very cloudy, and only few data could be obtained. All along the first campaign of 2005 a problem of astigmatism was detected. The beam-splitting calcites - Savart plate - of the $V$ polarimeter, mounted in front of the detector in the convergent beam, generate by principle a small astigmatism in the extraordinary beams. The orientations of the astigmatism are perpendicular for the two channels, $\frac{1}{2}(I+V)$ and $\frac{1}{2}(I-V)$. Now, the optical components between KAOS and the FPI spectrometer also generated an astigmatism which, incidentally, doubled the astigmatism in one polarimetric channel and compensated it in the other. At high spatial resolution, the polarimetric measurements were seriously affected. In spite of the a posteriori work trying to compensate this effect combining the point spread functions (PSFs) of both channels, reliable polarimetric signals could not be obtained due to this different astigmatism. During the other campaigns, a feature of KAOS could be used which allows a pre-setting of aberrations of the deformable mirror. This way the astigmatism of the optics between KAOS and the spectrometer could be compensated, and the detector of the spectrometer could be placed at the position of the circle of least confusion.

Taking advantage of the new possibility of observing in various wavelength ranges, one filter for observations in the Fe I $6173 \AA$ and another one for $\mathrm{H} \alpha(6563 \AA)$ were chosen. This provided information about the photosphere from a magnetically sensitive Fe I line - Landé factor $g=2.5$ - and about the chromosphere from $\mathrm{H} \alpha$. Table 3.2 presents the main parameters chosen for the observations.

Table 3.2: Main characteristics of FPI observations: date of campaign, spectral lines observed, number of spectral positions scanned, number of frames taken at each spectral position, step width between positions, and exposure time of each frame.

\begin{tabular}{cccccc} 
Campaign & Lines & Positions & Images/pos. & Step width $(\mathrm{m} \AA)$ & Exposure time $(\mathrm{ms})$ \\
\hline $04 / 05.2005$ & $6173 \AA$ & 21 & 15 & 23.65 & 5 \\
08.2005 & $6173 \AA+6563 \AA$ & $21 / 22+23$ & 15 & $23.65+100.67$ & $5+10$ \\
04.2006 & $6173 \AA+6563 \AA$ & $22+23$ & 15 & $23.65+100.67$ & $5+10$
\end{tabular}

In order to perform correct speckle reconstructions (cf. Sect. 4.2), the wavelength of the broadband images must be close to the wavelengths selected for the narrow-band observations. Here, a 6300 A filter with a FWHM of $50 \AA$ 皮 was used. 
and May 2007. The weather and seeing conditions were very variable. At the beginning of the 2005 campaign, the passage of the famous Delta storm caused damages and problems in the telescope control. After a few days of repairing and resetting everything, the sky was clear, so the seeing was stable although not very good on average. During the 2006 campaign the sky was much more clouded making observations extremely difficult. Few data were obtained, with not very good seeing. Thus, KAOS could not lock very well onto PFe and did not operate at its best performance. The last campaign, in 2007, also contained many days with overcast sky. Yet on few very worthwhile days observations could be performed and good data could be acquired.

Throughout all TIP II campaigns, the range at $1.56 \mu \mathrm{m}$ was observed, focusing on the two Fe I lines at $15648 \AA$ and $15652 \AA$ with the set of liquid crystals appropriate for that range. The sets of parameters chosen for the observations are given in Table 3.3

Table 3.3: Parameters of TIP II observations: date of campaigns, size of used slit, number of accumulations in each position, exposure time for each of those positions, and step size from one position to the next.

\begin{tabular}{ccccc} 
Campaing & Slit width $(\mu \mathrm{m})$ & Accumulations & Exposure time $(\mathrm{ms})$ & Step size $\left({ }^{\prime \prime}\right)$ \\
\hline $11 / 12.2005$ & 100 & 5 & 250 & 0.5 \\
10.2006 & 100 & 5 & 250 & 0.35 \\
05.2007 & 60 & 5 & 250 & 0.35
\end{tabular}

The number of spatial positions scanned for each observation ranged from 15 to 120 , depending on the amount and size of PFe visible in the FOV. These various spatial extensions contain from just one or two PFe to many of them as well as quiet Sun allowing for some magnetic flux measurements outside PFe.

The step size of $0.35^{\prime \prime}$ corresponds approximately to twice the pixel size along the slit, which is $0.17^{\prime \prime}$, making it easy with an interpolation to have the same scaling in both slit and scanning direction. Likewise, the step of $0.5^{\prime \prime}$ corresponds closely to three times the pixel size along the slit, so again a simple interpolation gives the same scaling in both directions.

Simultaneously with the spectropolarimetric observations, slit jaw images were recorded. Mainly white light images were taken, but also some images in calcium $\mathrm{K}$ and some in $\mathrm{H} \alpha$ with the Lyot filters of the VTT's slit jaw camera. They serve as information in different wavelengths about the solar area around the positions from where spectropolarimetric data with the slit spectrograph were taken.

The usual flat field and calibration data were also obtained in order to perform later the data reduction, explained in Chapter 4 


\title{
4 Data Reduction
}

"In the absence of light, darkness prevails."

Hellboy (2004)

\begin{abstract}
"Data reduction" refers to the application of techniques for "cleaning" the data, for removing noise and imperfections which do not belong to the true object under study but result from the way the data were obtained. Earth's atmosphere, dust in the instruments, inexact telescope guiding and telescope jitter, instrumental polarisation, etc. are sources for these imperfections.

This chapter will describe some of the steps to be undertaken before the actual work with the data, to have them prepared for scientific interpretation. In Sect. 4.1 the standard processing will be described. Sect. 4.2 deals with the speckle reconstruction, which was mentioned above in Sect. 3.1.2 as a way to improve the spatial resolution of the observations. In Sect. 4.3 the different methods used in the subsequent work to obtain magnetic and velocity fields will be presented.
\end{abstract}

\subsection{Reduction}

For the observations from both FPI and TIP II instruments, the basic reduction processes concerning the dark correction and flat fielding, were performed in the usual way. Dark frames and flat field exposures were obtained during the observations and are used as explained in the following.

Darks are applied to take into account the number of counts that detectors are delivering just by being in operation. An image with the same exposure time as the data, but with the light blocked, is recorded to measure the thermal counts and any electronic bias. This offset is then removed from all the data, including the flat fields.

Flat fields allow to measure the different response of each pixel of the detector. This includes the variations of the pixel sensitivity (or gain table), vignetting, and shadows from dust on the optical surfaces. To quantify these fluctuations, the detector is exposed to a homogeneous constant intensity (flat field). On the Sun, a homogeneous field does not exist since there are always spatial structures such as granulation. Thus, to correct for this effect, several images are taken whilst changing the telescope pointing in a random way near the disc centre of the Sun. These several flat field images are then averaged in order to smooth out the intensity variation due to spatial structures of the images, and to obtain the required homogeneous field. All data will then be corrected for the remaining pattern. 
In summary, the dark and flat field corrections are applied as

$$
\text { data reduced }=\frac{\text { original data }- \text { dark }}{\text { flat field }- \text { dark }}
$$

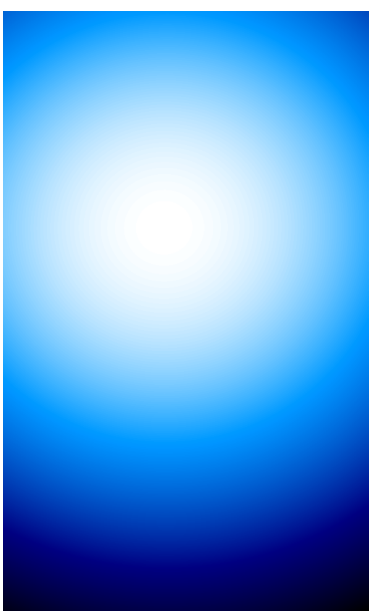

Figure 4.1: Example of the induced blueshift from a flat field scan in one narrow-band channel.
Special care must be taken with the reduction of the narrow-band FPI data, regarding the previously mentioned (Sect. 3.1.2) blueshift induced by the mounting of the FPI. An example of such a blueshift is presented in Fig. 4.1 The image shows the pattern of the blueshift from a narrow-band flat field scan. The brightness variation in Fig. 4.1 corresponds to the different amount of displacement of the FPI transmission in wavelength. It is calculated just by measuring the difference between the position of the line minimum in each pixel with respect to the position of the line minimum of the mean profile. As the flat field scans do not contain any solar line shifts, the blueshift pattern is obtained from them, and used for the wavelength correction of the line profiles across the FOV.

The blueshift correction should be applied to the data from the narrow-band train, both flat field images and science data - separately for each narrow-band channel - because flat fields and science data are equally affected.

A continuum correction is also applied to the data from both instruments, FPI and TIP II, to rectify the variation of continuum intensity with wavelength. This effect results from the transmission curve of the order sorting interference pre-filters. The continuum correction is performed differently for each instrument.

In the case of the FPI, the detector is illuminated with light from a halogen lamp, which emits homogeneous continuum light. These so-called "continuum images" and "continuum scans", are recorded additionally in the course of the observations.

For TIP II there is no halogen lamp from which to calibrate the influence of the pre-filter. However, the high spectral range covered by TIP II allows to select several continuum windows at different wavelengths. A polynomial is then fitted to these wavelength areas to estimate the filter's transmission curve. Dividing by the latter leaves a constant continuum level throughout the whole spectral range. Figure 4.2 depicts an original spectrum along with the continuum corrected spectrum and the one from the FTS atlas. The continua of both the corrected and the atlas profile coincide to a large extent. It was stated above in Sect. 3.1.2, that the FTS atlas can be considered as free of instrumental broadening. Since it can be seen from Fig. 4.2 that the corrected spectrum compares well with atlas data, the corrected data can be considered as almost free of instrumental broadening as well.

There are some more steps in the TIP II data reduction that should be mentioned. 


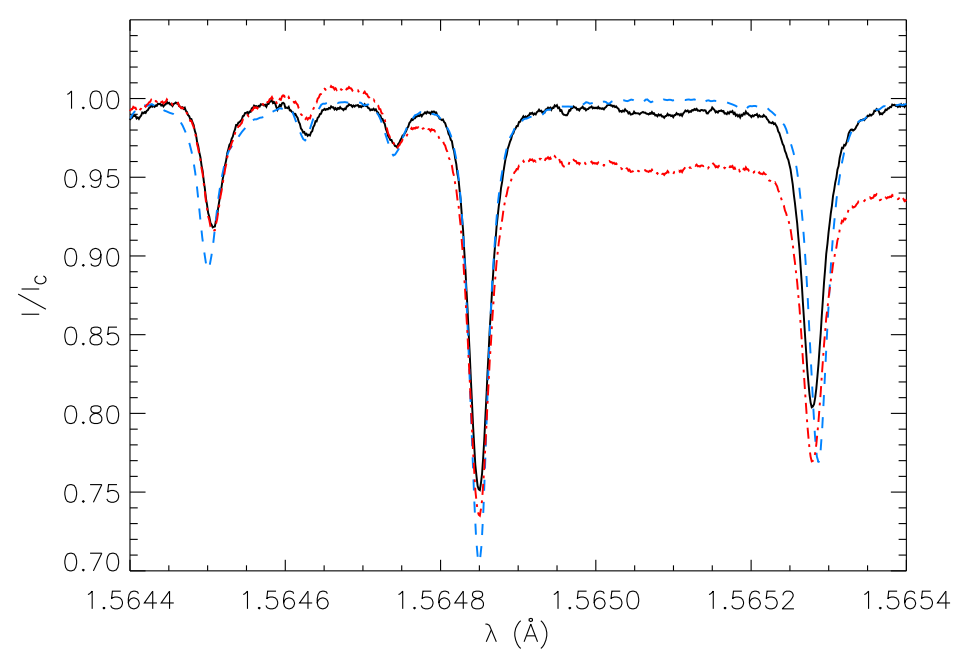

Figure 4.2: Flat field spectrum before (dash-dotted red line) and after (solid line) continuum correction. The dashed blue line shows the spectrum of the atlas by Livingston \& Wallace (1991). The original data have been scaled to their maximum intensity.

Most of the TIP II reduction procedures are already implemented in programs written and supplied by M. Collados (Instituto de Astrofísica de Canarias, Tenerife, Spain). These programs use the dark, flat field, and calibration data taken during the observations as well as combine the two beams and four states of the data, in order to perform the dark and flat field corrections, take out the signature of some bad pixels and remove the crosstalks, i.e. the polarisation induced by the optical elements.

Still, unreasonable intensities, and some residual noise or periodic signals in wavelength with short periods, in some bad pixels remained. To correct for these last disturbing effects two more procedures were applied. Firstly, the remaining bad pixel intensities were interpolated with their neighbouring ones. Then, a low-pass filter was applied to the spectra for smoothing and reducing the noise.

One important pre-analysis measure is the wavelength spectral sampling as it will be involved in the calculations of the magnetic field strengths and velocities. In the FPI case the step size is chosen by the observer so it does not require any further work. For TIP II, the difference between the tabulated wavelength positions of the iron lines was compared with the observed difference. This yielded a step width corresponding to $14.4 \mathrm{m \AA} / \mathrm{pixel}$.

\subsection{Speckle reconstruction}

Even with the use of an excellently working AO system, most of the time the Earth's atmosphere impedes achieving diffraction-limited resolution in the observations. But the pursuit of resolving the smallest structures on the Sun requires further improvements to reduce the degradation of observations. Therefore, a combination of $\mathrm{AO}$ with some post factum - after the acquisition of the data - image reconstruction technique is needed. Here, speckle techniques have proved highly successful.

The name speckle comes from an intensity pattern created by mutual interference of 
coherent wavefronts with a difference in their phases - e.g., a laser reflected by a rough surface. Such a laser pattern is presented in the left frame of Fig. 4.3
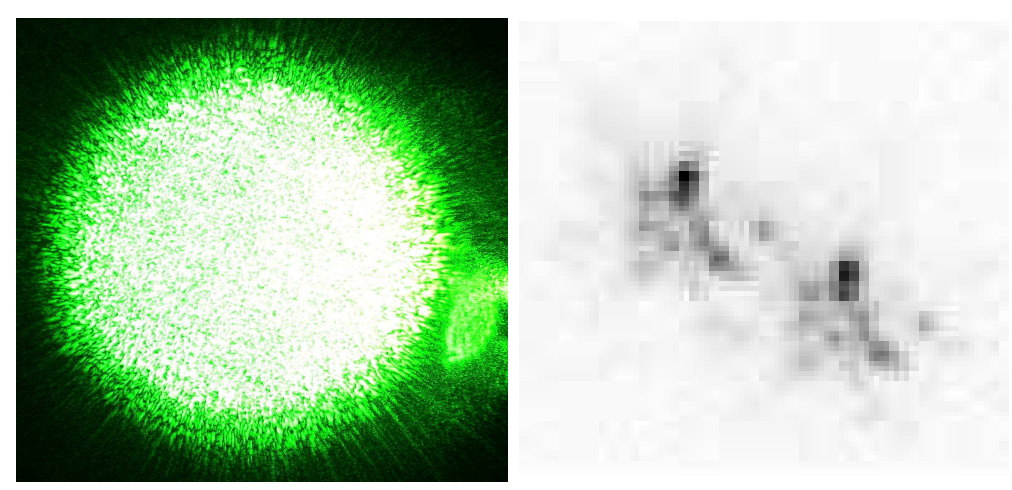

Figure 4.3: Examples of speckle pattern, from a laser (left) and from the $\zeta$ Bootis binary system (right). The $\zeta$ Bootis observation was made with the Nordic Optical Telescope (NOT) in May 2000. The laser pattern image is taken from user jurvetson on www.flickr.com.

Consider a star which, at Earth's distance, can be considered as a point source. A groundbased long-exposure image of the star appears as a diffuse intensity pattern, the seeing disc. But if observed with short exposure, it reveals a speckle pattern which changes and moves globally in time. The exposure time must be shorter than the time scale of atmospheric variations, typically a few 10 milliseconds. The atmosphere would then be frozen. An example of such circumstances is shown in the right frame of Fig. 4.3 with an observation of the $\zeta$ Bootis binary system.

Speckle images are taken with these short time scales. Thus, each of them is only affected by the instantaneous atmosphere. Then a statistical approach can be made. Many - of the order of 100 or more - speckle images are taken so the arithmetic averages used below will be approximately equal to ensemble averages. From here the atmospheric conditions can be inferred and the seeing degradation can be reduced.

An image taken through an optical device and medium - e.g. telescope and atmosphere - can be expressed mathematically as the following convolution

$$
i(\vec{x})=o(\vec{x}) \otimes p s f(\vec{x}),
$$

where $i$ is the image at the detector, $o$ is the original object and psf is the point spread function (PSF), which contains the information about the medium and the device.

Considering a series of $\mathrm{N}$ images and changing to the Fourier space, their average is

$$
\frac{1}{N} \sum_{i=1}^{N} I_{i}(\vec{q})=O(\vec{q}) \frac{1}{N} \sum_{i=1}^{N} O T F_{i}(\vec{q})
$$

being $I, O$, and the optical transfer function $(O T F)$ the Fourier transforms of $i, o$ and $p s f$. It can be seen from the last equation that the loss of spatial resolution, when dealing with a long exposure image, $\sum_{i} I(\vec{q})$, comes from the summation of the OTFs. Due to the changing atmosphere the complex Fourier components are cancelled, upon summation of the instantaneous OTFs. Thus, information at high spatial frequencies is lost. The effort 
shall be put then in the recovery of this information, both on amplitudes and on phases. To this aim the speckle reconstruction code was developed at the Universitäts-Sternwarte Göttingen (de Boer 1996). The idea of the code is the separate recovery of the amplitudes and the phases. For the phases, the so-called speckle masking method (Weigelt 1977) is applied. For the amplitudes, starting from Labeyrie's idea (Labevrie 1970), the spectral ratio method (von der Lühe 1984) is used.

To avoid the cancellation and to recover the amplitudes, Labeyrie proposed to take the square modulus of the Fourier components in the average,

$$
\frac{1}{N} \sum_{i=1}^{N}\left|I_{i}(\vec{q})\right|^{2}=|O(\vec{q})|^{2} \frac{1}{N} \sum_{i=1}^{N}\left|O T F_{i}(\vec{q})\right|^{2} .
$$

With this approach the speckle transfer function $\mathrm{STF}=\frac{1}{N} \sum_{i=1}^{N}\left|O T F_{i}(\vec{q})\right|^{2}$, does not cancel out at high spatial frequencies.

Since the real object is unknown - and, therefore, also its Fourier transform $O(\vec{q})$ - von der Lühe set forth the spectral ratio method, dividing the squared modulus of the averaged Fourier transforms by the average power spectrum:

$$
\epsilon(\vec{q})=\frac{|\langle I(\vec{q})\rangle|^{2}}{\left\langle|I(\vec{q})|^{2}\right\rangle}=\frac{|O(\vec{q})|^{2}}{|O(\vec{q})|^{2}} \frac{|\langle O T F(\vec{q})\rangle|^{2}}{\left\langle|O T F(\vec{q})|^{2}\right\rangle} .
$$

This ratio depends only on the telescope and atmospheric conditions and is characterised by the normalised Fried parameter $\alpha=r_{0} / D$, where $D$ is the diameter of the telescope's entrance pupil and $r_{0}$ is the Fried parameter.

In order to obtain $\epsilon$ and, from that, $r_{0}$, the observed and the theoretical ratio $\epsilon(\vec{q})$ are compared. The theoretical expressions of the averages in Eq. 4.5] are based on work by Fried (1966) for $|\langle O T F(\vec{q})\rangle|^{2}$ and by $\operatorname{Korff}(1973)$ ) for the STF. With this information, the STF corresponding to the observations is known and, from Eq. 4.4 the amplitudes of the object are obtained.

The speckle masking method uses the bispectrum to retrieve the still missing phases. The bispectrum is defined as

$$
B S(\vec{q}, \vec{p})=\langle I(\vec{q}) I(\vec{p}) I(-\vec{q}-\vec{p})\rangle .
$$

Considering the image as a two-dimensional pixel matrix and using Eq. 4.3, Eq. 4.6 can be re-written as

$$
\begin{aligned}
B S(i, j, k, l) & =O(i, j) O(k, l) O(-i-k,-j-l) \\
& \times\langle\operatorname{OTF}(i, j) O T F(k, l) O T F(-i-k,-j-l)\rangle .
\end{aligned}
$$

It can be proven that the bispectrum of the OTF, upon sufficient averaging, gives a real non-zero function. This means that its phases are zero and therefore, $B S$ contains the phases of only the original object.

Applying the closure equation to the phases in Eulerian formulation, yields

$$
e^{i \phi(i+k, j+l)}=e^{i \phi(i, j)} e^{i \phi(k, l)} e^{-i \Phi(i, j, k, l)},
$$


where $\Phi$ is the phase of the bispectrum. Now, a recursive calculation starts from the initial condition $\phi(0,0)=0$ and from known phases at low frequencies, i.e. at large scales. From here, all the phases will be recovered.

All this processing requires an instantaneous OTF that does not vary over the FOV. But this latter is correct only for an isoplanatic patch, mentioned above in Sect. 3.1.1. The size of an isoplanatic patch is of the order of 5", much smaller than the FOV of the FPI. The FOV is hence divided into subfields with the approximate size of the isoplanatic patch and the speckle reconstruction is applied to each of them. Subsequently, the subfields are reassembled again.

Figure 4.4 presents an example of an image before and after the speckle reconstruction. The uppermost image is the best - i.e., the one with highest contrast - of the speckle images of a burst of 330 frames. The middle image shows an average over all the images from the burst, corresponding thus to a long exposure image. The small details are clearly blurred. The lowest image is the speckle reconstructed image. The contrast is highly enhanced and the small details are better defined than in the upper image.

Recently, the speckle reconstruction code was improved by Puschmann \& Sailer (2006) to take into account one further effect introduced by the use of AO.

In Sect. 3.1.1 the problem of the field dependence of the AO correction was pointed out. This leads to a radial variation of the spectral ratio whereas in the previous calculations $\epsilon$ was only determined by $r_{0} / D$. The solution was to divide the FOV into a circle centred on the KAOS lockpoint and 6 further concentric annuli. The diameter of the central circle and the width of the annuli are the same. In each of these annuli, the spectral ratio is assumed constant and is estimated independently. Only the subfields whose centre lies within an annulus are used for the calculation of $r_{0}$ in that specific annulus.

Narrow-band reconstruction. Albeit much information on the structure of the solar atmosphere is contained in the speckle reconstructed broadband images, the information that can be obtained from the narrow-band spectropolarimetric scans is much higher. As was described in Sect. 2] spectropolarimetry gives the opportunity of analysing many characteristics of the region under study unaccessible by other methods. Thus the reconstruction of the narrow-band images - i.e., the improvement of their spatial resolution is as important as the broadband reconstruction.

In the narrow-band case, the same speckle reconstruction method applied to the broadband would bring serious constraints. Taking a few hundred images at each spectral position creates problems not only at a storage capacity level, but also of time resolution. One scan would take up to 7 minutes from start to end. Most of the time scales on the Sun which are of interest here, are shorter than this time, so the structures under study would have undergone a complete change during the scan.

This is the main reason to have a broadband and a narrow-band train operating in parallel. The observations in both bands are taken simultaneously so they are affected by the same atmospheric perturbations. Once these perturbations are known and removed from the broadband images, that information can be used for the narrow-band reconstruction, applying the method by Keller \& von der Lühe (1992).

The observations are expressed as, again in Fourier space,

$$
I_{\mathrm{bb}, i}=O_{\mathrm{bb}} O T F_{i}
$$



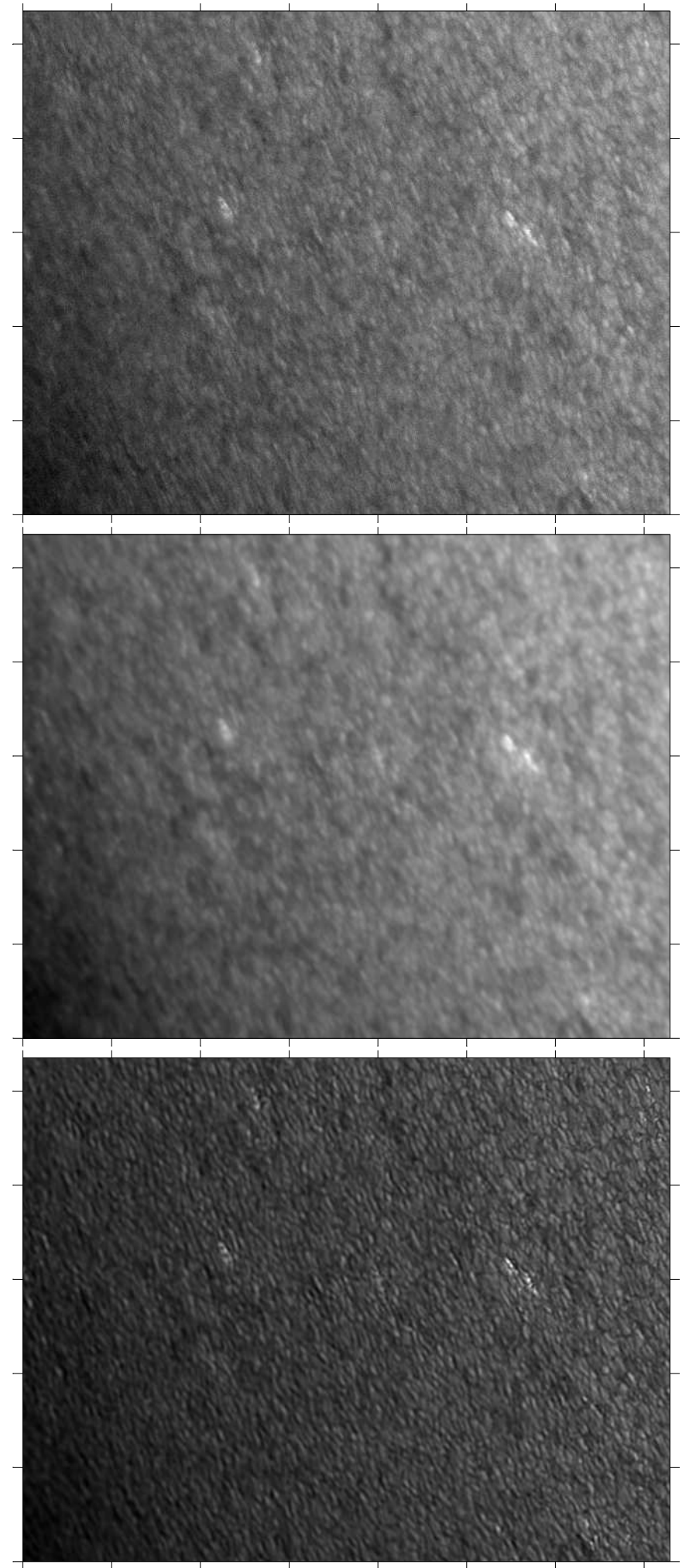

Figure 4.4: Speckle reconstruction of a FOV showing an original short exposure speckle image (top), an average of the 330 speckle images (middle) and the actual speckle reconstructed image (bottom). Tickmark intervals correspond to $10^{\prime \prime}$. The observations for this figure were obtained in May 2005. 


$$
I_{\mathrm{nb}, i}=O_{\mathrm{nb}} O T F_{i},
$$

where the index 'bb' refers to the broadband train and the index 'nb' to the narrow-band train. The two objects, $O_{\mathrm{bb}}$ and $O_{\mathrm{nb}}$, are different as they correspond to different heights in the solar atmosphere. But the OTFs in Eqs. 4.9 and 4.10 are the same, provided that the spectral regions used for the broad and narrow-band observations are close enough. Thanks to the speckle reconstruction described above, the OTFs as well as the estimate of the broadband object are known. From Eqs.4.9 and 4.10 one obtains upon averaging

$$
O_{\mathrm{nb}}=\left\langle\frac{I_{\mathrm{nb}, i}}{O T F_{i}}\right\rangle=\left\langle\frac{I_{\mathrm{nb}, i}}{I_{\mathrm{bb}, i}}\right\rangle O_{\mathrm{bb}} .
$$

This estimation can be very noisy because some of the $O T F_{i}$ may have values close to zero at high spatial frequencies. To have a better estimate, Eq. 4.11 is weighted with the power spectrum of $I_{\mathrm{bb}, i}$,

$$
O_{\mathrm{nb}}=\frac{\left\langle\left(I_{\mathrm{nb}, i} / I_{\mathrm{bb}, i}\right)\left|I_{\mathrm{bb}, i}\right|^{2}\right\rangle}{\left\langle\left|I_{\mathrm{bb}, i}\right|^{2}\right\rangle} O_{\mathrm{bb}}=\frac{\left\langle I_{\mathrm{nb}, i} I_{\mathrm{bb}, i}^{*}\right\rangle}{\left\langle\left|I_{\mathrm{bb}, i}\right|^{2}\right\rangle} O_{\mathrm{bb}} .
$$

Note that Eq. 4.12 can also be obtained from a least-square calculation, i.e. by minimising the error metric

$$
E=\sum_{i}\left|O_{\mathrm{nb}} \frac{I_{\mathrm{bb}, i}}{O_{\mathrm{bb}}}-I_{\mathrm{nb}, i}\right|^{2}
$$

A further reduction of noise was achieved by applying an optimum filter $H$,

$$
O_{\mathrm{nb}}=H \frac{\left\langle I_{\mathrm{nb}, i} I_{\mathrm{bb}, i}^{*}\right\rangle}{\left\langle\left|I_{\mathrm{bb}, i}\right|^{2}\right\rangle} O_{\mathrm{bb}}
$$

The level of noise in the data, used to create the filter, is estimated from defocused flat fields. These are images taken under the same conditions as the data but with the telescope out of focus (see e.g. Koschinsky 2001).

The narrow-band reconstruction process is applied to the data from both channels of the polarimeter in the same way.

The last step before further analysis of the data is performing a careful, sub-pixel accurate alignment of the images from both channels of the $V$ polarimeter, and of these images with the broadband images.

As noted in Sect. 3.1.2 the two channels of the $V$ polarimeter give the two circularly polarised states of light, $\frac{1}{2}(I+V)$ and $\frac{1}{2}(I-V)$. To obtain images of Stokes $I$ and $V$ as parameters to be analysed, one has to add and subtract the images from the two channels of the polarimeter. Therefore, an alignment of the data from the two channels must be carried out to avoid false signals. Likewise, some of the later analyses will require studying together information obtained simultaneously from the narrow-band and broadband channels. Thus, the alignment of images from both bands is also necessary.

The alignment process consists of a global shift for maximum correlation and of a destretching with an extension of the code by Yi \& Molowny Horas (1992). This de-stretching corrects small displacements which vary across the FOV, due to different optical paths of 
the two polarised light channels and the broadband optical train. The de-stretching parameters are calculated from averaged continuum images in the narrow-band channels. The parameters so calculated are then applied to the reconstructed images at all wavelength positions of the scanned line profile.

\subsection{Magnetic field and velocity determination}

Once the data are freed from imperfections as much as possible - and reconstructed, in the FPI case - the next step is to obtain from them physical parameters of the solar atmosphere to infer the magnetic and velocity fields in the observed area on the Sun from the properties of the measured intensities, including polarisation.

Section 2.3 described the response of the Zeeman splitting to the magnetic field strength and the polarisation of the different Zeeman components. The different methods used in the present work to translate the response of the measured spectropolarimetric properties - such as separation and amplitudes of Stokes $V$ extrema and line shifts - into the magnetic and velocity fields generating them, are discussed in this Section. Also a comparison of the different methods using synthetic Stokes profiles is carried out (cf. Fig. 4.7).

The magnetic field strength was measured by three methods: weak field approximation (WFA), strong field regime (SFR), and centre of gravity (COG). The former two methods extract the field strengths from the Stokes $V$ profiles whereas the COG method uses the profiles corresponding to the two circular polarisation states $\frac{1}{2}\left(I_{\lambda}+V_{\lambda}\right)$ and $\frac{1}{2}\left(I_{\lambda}-V_{\lambda}\right)$.

Weak Field Approximation. In the WFA, the Zeeman splitting, $\Delta \lambda_{B}$ (cf. Eq. 2.13), is assumed to be much smaller than the Doppler width, $\Delta \lambda_{D}$, of the line

$$
\Delta \lambda_{B}<<\Delta \lambda_{D}=\sqrt{\frac{2 k T}{m_{A}}} \frac{\lambda}{c}
$$

where $k$ is the Boltzmann constant, $T$ the gas temperature and $m_{A}$ the atomic mass. In this case, the amplitudes of the Stokes $V$ profiles increase linearly with the LOS component of the magnetic field, as seen in Fig. 2.5 b. From the expansion of the terms in the equation of transfer of polarised light in a Taylor series to first order in $\Delta \lambda_{B}$ (Landi Degl'Innocenti 1992), one obtains

$$
V(\lambda)=-4.67 \times 10^{-13} g_{\text {eff }} \lambda_{0}^{2} \bar{B}_{\text {eff }} \frac{\mathrm{d} I_{0}(\lambda)}{\mathrm{d} \lambda},
$$

where $I_{0}(\lambda)$ is the non-split Stokes $I$ profile, $\bar{B}_{\text {eff }}=\alpha \cos \gamma \bar{B}$ is the LOS component of the flux density averaged over the resolution element (and over the formation heights), with $\gamma$ the angle between the LOS and $\vec{B} . \alpha$ is the filling factor (cf. Sect. 2.3.

A low filling factor $\alpha<1$, e.g. due to limited spatial resolution, results in decreased amplitudes of Stokes $V$. In the case of very small $V$ signals, they become buried in the noise. Given the characteristic shape of Stokes $V$ in the presence of magnetic fields, the observed profiles were fitted by a non-linear least-squares method (minimising $\chi^{2}$ ) to two 
Gaussians,

$$
\chi^{2}=\sum_{i}\left[V_{\mathrm{obs}}\left(\lambda_{i}\right)-F\left(\lambda_{i}\right)\right]^{2},
$$

where

$$
F\left(\lambda_{i}\right)=A_{1} e^{-\left(\lambda_{i}-B_{1}\right)^{2} / 2 C_{1}^{2}}+A_{2} e^{-\left(\lambda_{i}-B_{2}\right)^{2} / 2 C_{2}^{2}} .
$$

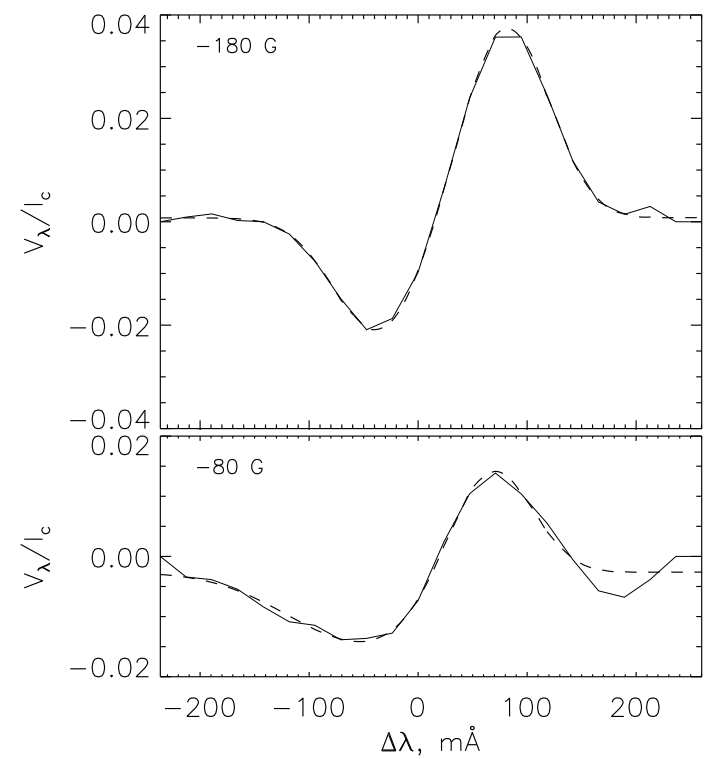

Figure 4.5: Examples of observed Stokes $V$ profiles (solid lines) and fits with two Gaussians (dashed lines). The resulting field strengths are $-180 \mathrm{G}$ (upper panel) and $-80 \mathrm{G}$ (lower panel).
The fit increases the polarimetric sensitivity and allows to distinguish magnetic signals from the background noise. The amplitudes of Stokes $V$ were calculated from the amplitudes of the Gaussian fit in the pixels where it worked properly. Figure 4.5 depicts two examples of Stokes $V$ profiles from data observed during the August 2005 campaign (cf. Table 3.2) and their corresponding Gaussian fits. The field strengths resulting from the profiles are $-180 \mathrm{G}$ and $-80 \mathrm{G}$ for the upper and lower panel of Fig.4.5, respectively, calculated from the amplitudes of the Gaussian fits.

\section{As also explained in Landi Degl'Innocenti} (1992), the Stokes parameters for linear polarisation, $Q$ and $U$, are often related (although without solid physical justification)

to the second derivative of the intensity and can be expressed as

$$
\begin{aligned}
Q(\lambda) & =-\frac{1}{4}\left(g_{\text {eff }} C \lambda_{0}^{2} B\right)^{2} \sin ^{2} \gamma \cos 2 \chi \frac{d^{2} I_{0}(\lambda)}{d \lambda^{2}} \\
U(\lambda) & =-\frac{1}{4}\left(g_{\text {eff }} C \lambda_{0}^{2} B\right)^{2} \sin ^{2} \gamma \sin 2 \chi \frac{d^{2} I_{0}(\lambda)}{d \lambda^{2}},
\end{aligned}
$$

with $C=4.67 \times 10^{-13} \mathrm{G}^{-1} \AA^{-1}$. Combining these two expressions of $Q$ and $U$, the azimuthal angle and the transversal component of the magnetic field are calculated

$$
\begin{array}{r}
\chi=\frac{1}{2} \arctan \frac{U}{Q}, \\
B_{\text {trans }}=\frac{1}{D}\left(Q^{2}+U^{2}\right)^{\frac{1}{4}},
\end{array}
$$

where $D=\left[\frac{1}{16}\left(g_{\mathrm{eff}} C \lambda_{0}^{2}\right)^{4}\left(\frac{d^{2} I_{0}}{d \lambda^{2}}\right)^{2}\right]^{1 / 4}$.

In addition to using the two-Gaussians fit, which does not work properly at many pixels in the FOVs since the fitting procedure does not find reasonable values, a different 
approach was also performed. A least-squares calculation from Eq.4.16yields

$$
\bar{B}_{\mathrm{eff}}=\frac{\sum_{i} V\left(\lambda_{i}\right) C^{\prime}\left(\lambda_{i}\right)}{\sum_{i} C^{\prime 2}\left(\lambda_{i}\right)}
$$

where $C^{\prime}=-4.67 \times 10^{-13} g_{\text {eff }} \lambda_{0}^{2} d I_{0}\left(\lambda_{i}\right) / d \lambda$.

Likewise, a least-squares calculation from Eqs. 4.19 and4.20yields

$$
\begin{gathered}
B_{\text {trans }}^{2}=\frac{\sum_{i} Q\left(\lambda_{i}\right) D_{1}^{\prime}\left(\lambda_{i}\right)}{\sum_{i} D_{1}^{\prime 2}\left(\lambda_{i}\right)} \\
B_{\text {trans }}^{2}=\frac{\sum_{i} U\left(\lambda_{i}\right) D_{2}^{\prime}\left(\lambda_{i}\right)}{\sum_{i} D_{2}^{\prime 2}\left(\lambda_{i}\right)},
\end{gathered}
$$

with $D_{1}^{\prime}=-\frac{1}{4}\left(g C \lambda_{0}^{2}\right)^{2} \cos 2 \chi \frac{d^{2} I_{0}}{d \lambda^{2}}$ and $D_{2}^{\prime}=-\frac{1}{4}\left(g C \lambda_{0}^{2}\right)^{2} \sin 2 \chi \frac{d^{2} I_{0}}{d \lambda^{2}}$. Like in Eq. 4.22, the addition of the squares of Eqs. 4.24 and 4.25 must be performed in order to eliminate the unknown angle $\chi$.

In this study, the summations in Eqs.4.23 4.24 and 4.25 included all wavelengths in the scanned line profile. These determinations of $\bar{B}_{\text {eff }}$ and of $B_{\text {trans }}$ worked at all pixels in the FOVs.

Strong Field Regime. For strong fields and/or magnetically very sensitive lines the SFR applies. As shown in Fig. 2.5, at strong fields, the amplitudes of Stokes $V$ are saturated. They do no longer augment with the field strength, whereas the two lobes become increasingly separated. In this regime, the Zeeman splitting is bigger than the Doppler broadening, $\Delta \lambda_{B}>\Delta \lambda_{D}$, as seen also in Fig. 2.5. The separation of the lobes, $\Delta \lambda_{V}$, approaches twice the Zeeman splitting and thus, the magnetic field can be calculated from

$$
\Delta \lambda_{V}=2 \Delta \lambda_{B}=2 C g_{\text {eff }} \lambda^{2} B
$$

In this way, the modulus of the magnetic field strength is estimated, independently of the angle $\gamma$. The advantage of using the separation of the Stokes $V$ lobes to calculate the magnetic field strength is that it is also not dependent on the filling factor. This latter decreases the amplitudes of Stokes $V$ but does not affect the location of the extrema.

The separations $\Delta \lambda_{V}$ were determined from the locations of the Stokes $V$ extrema, obtained by means of the same two-Gaussians fit as in the WFA.

Centre of Gravity. The actual intensities obtained after the $V$ polarimeter in the FPI do not correspond to Stokes $I$ and $V$ but consist of the two circularly polarised states of light. A way to derive the magnetic field directly from the $\frac{1}{2}(I+V)$ and $\frac{1}{2}(I-V)$ profiles has proven to be very robust and reliable. The COG method (cf. Semel 1967; Rees \& Semel 1979) obtains the LOS component of the magnetic field - averaged over the resolution element and formation heights - from the separation of the COGs of the two profiles 
$I_{+}=\frac{1}{2}\left(I_{\lambda}+V_{\lambda}\right)$ and $I_{-}=\frac{1}{2}\left(I_{\lambda}-V_{\lambda}\right)$. The COG of each of the profiles is defined as

$$
\lambda_{ \pm}=\frac{\int\left[I_{\mathrm{c}}-I_{ \pm}(\lambda)\right] \lambda \mathrm{d} \lambda}{\int\left[I_{\mathrm{c}}-I_{ \pm}(\lambda)\right] \mathrm{d} \lambda},
$$

with $I_{\mathrm{c}}$ being the continuum intensity and the integration being performed over the whole line profile. The calculation of the COGs is not affected by spectral broadening, e.g. by the spectrometer.

Using Eq. 2.13 and the above definition, the determination of the magnetic field is done with the relation

$$
\Delta \lambda_{B}=\frac{1}{2}\left(\lambda_{+}-\lambda_{-}\right)=4.67 \times 10^{-13} g_{\mathrm{eff}} \lambda_{0}^{2} \bar{B}_{\mathrm{eff}} .
$$

In Fig. 4.6 a section from a FOV containing various PFe is shown, as an example of the speckle reconstruction and of a magnetogram obtained via the COG method. The magnetogram only depicts effective fields of $\left|B_{\text {eff }}\right|>75 \mathrm{G}$ and both magnetic polarities are seen as bright and dark patches. The FOV is located at $\cos \theta=\mu=0.4$, where $\theta$ is the heliocentric angle. A spatial resolution of $0.4^{\prime \prime}-0.5^{\prime \prime}$ is estimated. Note in Fig. 4.6 the presence of magnetic features with no associated bright counterpart (upper-right area) and of brightnesses with no identifiable magnetic field (lower-left). Also note the existence of both positive and negative polarities with associated bright features. These points will be described more in-depth in Chapter [5]

Using the same assumptions for calculating synthetic profiles as in Sect. 2.3 and for Fig. 2.5- Milne-Eddington, Fe I $6173 \AA$ A, $30 \mathrm{~m} \AA$ Doppler width, static, constant magnetic field -, the previous methods for the magnetic field retrieval are tested. The results are shown in Fig. 4.7. Three different inclinations of the magnetic field with respect to the LOS are analysed: $\gamma=0^{\circ}$ (red) for the behaviour when the magnetic field is parallel to the LOS, $\gamma=50^{\circ}$ (yellow), and $\gamma=80^{\circ}$ (green) as the limits of the range of observations studied in this work. The dashed lines in Fig. 4.7 are the one-to-one correspondence taking into account the inclination for the magnetic field. As can be seen in Fig. 4.7, only the SFR gives the total field strength, not the LOS component. For low fields, the retrieval with this method is poor since it is the regime dominated by the amplitudes and the separation of the lobes does not change with field strength. Starting at approximately $1000 \mathrm{G}$, the measured separation of the lobes of Stokes $V$ behaves linearly with increasing field. For that and higher field strengths the measured strength becomes very similar to the introduced strength, with only a small overestimation.

Both the magnetic field obtained by means of a least-squares determination using Eq. 4.23 and the retrieval from the amplitudes of the two-Gaussians fit give very similar results to the introduced field strength until $B_{\text {eff }} \approx 500 \mathrm{G}$. The retrieval of the field strength is less accurate in the case of the WFA. The two methods respond linearly only at low field strengths. Beyond around $500 \mathrm{G}$, the amplitudes of Stokes $V$ saturate. From there and to higher strengths, the field measured under WFA comes to a constant value, while the strength determined with the least-squares starts to decrease. Since with increasing 


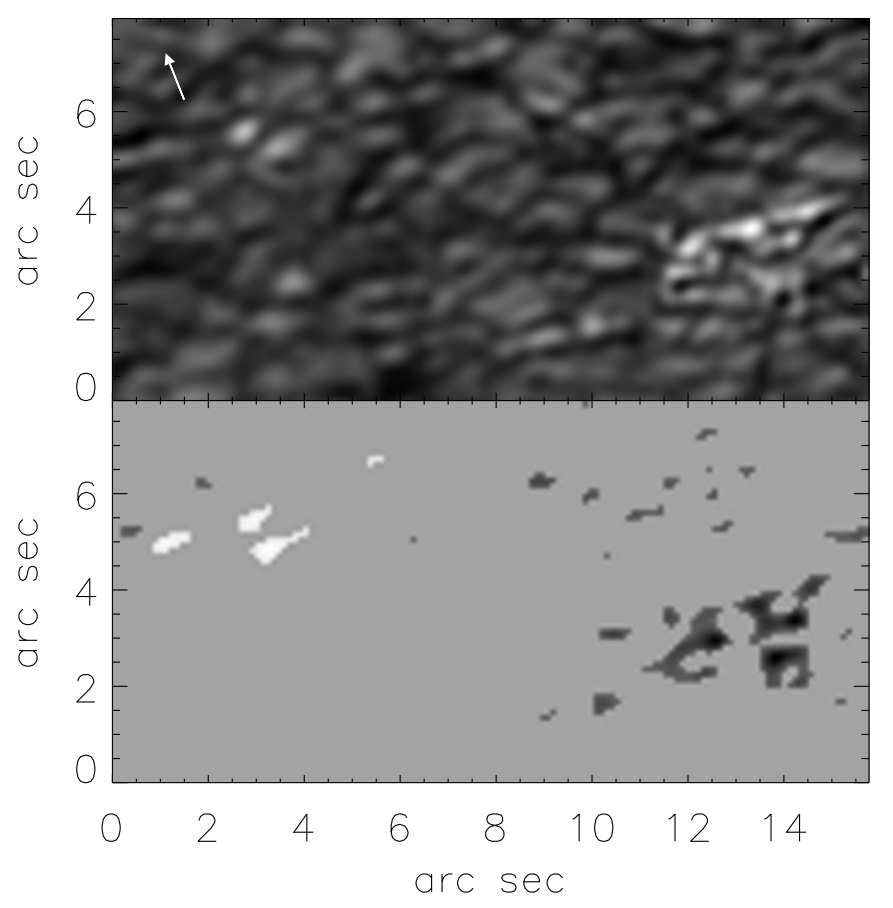

Figure 4.6: Example of PFe at $\cos \theta=\mu=0.4$. Upper frame corresponds to section of the speckle reconstructed image and lower frame to same section of magnetogram. In the magnetogram only fields of $\left|B_{\text {eff }}\right|>75 \mathrm{G}$ are drawn and opposite polarities marked as dark and bright patches. Arrow points towards limb. Observations for this figure were performed during the August 2005 campaign (cf. Table 3.2)
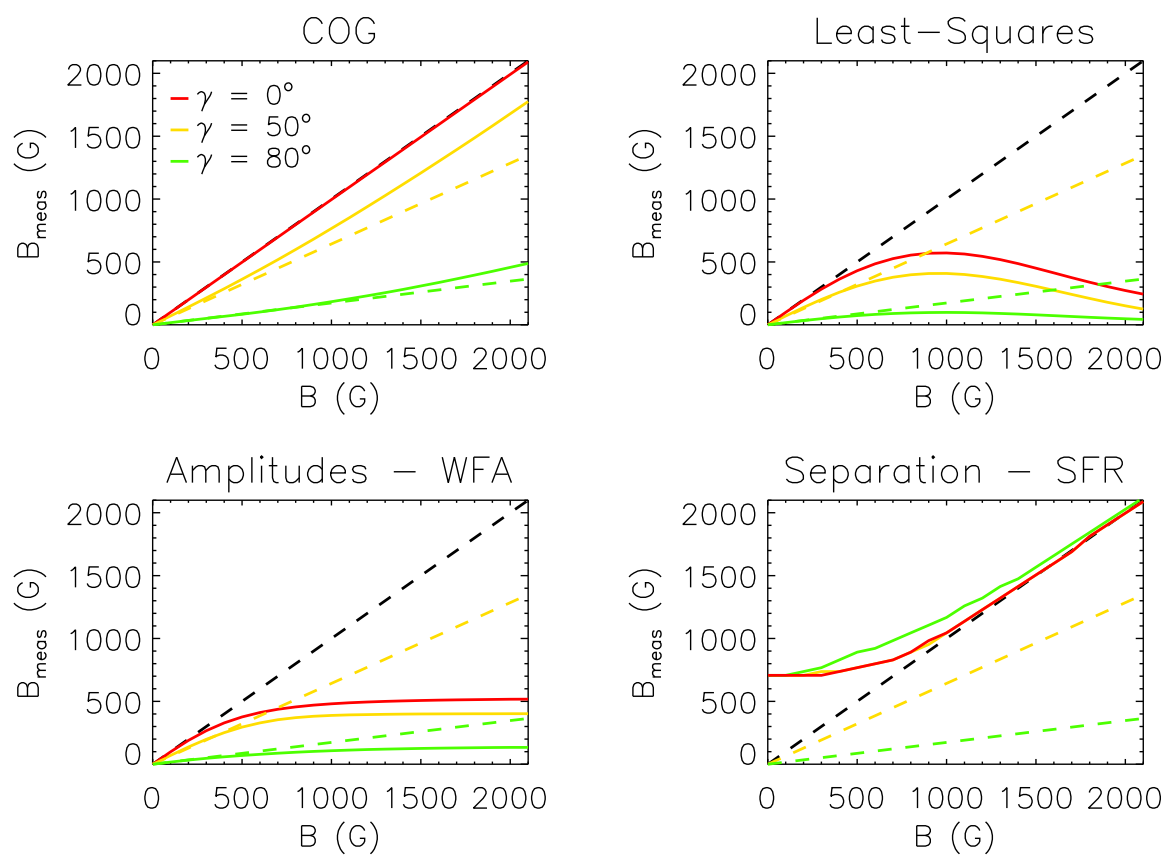

Figure 4.7: Retrieval of magnetic field $\mathrm{B}_{\text {meas }}$ with respect to the input field, $B$, from synthetic profiles. The dotted lines give the LOS component of the input field. Upper left: determination with COG method; upper right: least-squares determination with Eq.4.23, lower left: determination in WFA; lower right: assuming SFR. 
magnetic field strength, the lobes of Stokes $V$ start to separate whereas the derivative of the unperturbed Stokes $I$ does not change, the least-squares method using Eq. 4.23 gives decreasing strength of the field. The COG method responds more constantly to all applied field strengths than the other three methods, although for field strengths higher than approximately $1000 \mathrm{G}$ it overestimates the observed field for inclination angles $\gamma \neq 0^{\circ}$. For lower field strengths, the difference between the measured and the introduced field is lower than $10 \mathrm{G}$. Until approximately $500 \mathrm{G}$, the difference between the field strength obtained from the least-squares determination and the strength from the COG method is less than $20 \mathrm{G}$.

Velocities. Velocities in the atmosphere of the Sun can be obtained from the response of the spectropolarimetric signals to the various dynamic conditions of the plasma from which the radiation originated. In the present work, two methods were employed to measure the LOS velocities: the COG method and the Stokes $V$ zero-crossing.

From the two circularly polarised states, the Doppler shifts were measured by means of the average wavelength position of their COGs. As zero reference, the average velocity in the FOV was taken. This method gives the LOS velocity of magnetic and non-magnetic plasma in the resolution element averaged over the formation heights of the line.

The zero-crossing positions of the Stokes $V$ profiles were analysed with two methods, the above mentioned two-Gaussians fit to the $V$ profiles and a third order polynomial fitting the central part of the $V$ profiles, yielding very similar results. The output are maps of the LOS velocity of the magnetised plasma alone, since $V$ profiles are formed only in the presence of magnetic fields. These maps are not as continuous as the ones from COG. Whereas the profiles of $\frac{1}{2}(I+V)$ and $\frac{1}{2}(I-V)$ give a clear signal at every pixel, the Stokes $V$ signal is noisy, i.e. the zero-crossing is not measurable at positions with only weak fields.

Lambdameter Method. Velocities were also measured for the $\mathrm{H} \alpha$ data at different heights in the solar atmosphere, applying in this case the so-called "lambdameter method" (Tsiropoula et al. 1993). This method calculates the shifts in wavelength of the bisectors of each profile at certain line widths, with respect to the average profile of a quiet Sun region. Then, those shifts are related with Doppler shifts.

Instrumental Crosstalk. The instrumental crosstalk (cf. Sect. 2.2) is of concern here since it can affect the measurement of magnetic and velocity fields. Referring specifically to the methods described above, the determinations of $\bar{B}_{\text {eff }}$ and of $V$ zero-crossing velocities, are susceptible to instrumental crosstalk, especially to $Q, U \rightarrow V$ crosstalk.

For TIP II data, the double - spatial and temporal - modulation minimises the crosstalk induced by seeing and by uncertainties in the CCD's gain table (cf. Sect. 3.1.3). The crosstalk from the telescope and the AO is measured via the calibration optics in the course of the observations to remove it afterwards during data reduction. Unfortunately, for the FPI none of these possibilities were available - only since the last upgrade of the FPI (Bello González \& Kneer 2008), it is possible to measure both the full Stokes vector and the instrumental crosstalk, thus to correct for the latter.

An estimation of the crosstalk with the FPI was performed by Okunev (2004) and Bello González et al. (2005). The $Q, U, V \rightarrow I$ crosstalk is of negligible influence due to 
the low amplitudes of the polarisation signal compared to the intensity. The influence of $I \rightarrow V$ is also small and could be corrected during data analysis. However, the impact of $Q, U \rightarrow V$ distorts the Stokes $V$ profiles, leading to systematic errors in the determination of magnetic field strengths and velocities. The crosstalk increases towards the limb with increasing $Q$ and $U$. According to the estimates for the WFA and for the COG, $\bar{B}_{\text {eff }}$ comes out too small by $20 \%$ whereas the separations of the $V$ extrema, i.e. in the SFR, are little affected. But still, the velocities measured from the position of the zero-crossings have to be taken with caution. 



\title{
5 Results*
}

\author{
“- Inconceivable!
}

- You keep using that word. I do not think it means what you think it means.”

The Princess Bride (1987)

The favourable conditions during the realisation of this work - i.e., time of sunspot minimum, instrumental upgradings providing very high spatial resolution - allow to gain a deeper insight into the characteristics of PFe and their relation to the global magnetic field, based on statistical samples from many observed PFe. Analyses of the main properties of $\mathrm{PFe}$ and their variation with heliocentric angle are carried out, providing new information and constraints for future studies and numerical modelling. Comparisons between information obtained from the photospheric magnetic line Fe I $6173.3 \AA$ and the infrared lines at $1.56 \mu$ are carried out as well.

This Chapter presents the results obtained from the observations described in Chapter 3, by means of the methods explained in Chapter 4 The results will be discussed as well. Section 5.1 deals with photometric analyses of PFe observed in the broadband and continuum intensity maps, such as the number density, size distribution and contrast variation. Also the penetration of $\mathrm{PFe}$ into higher atmospheric layers is described with $\mathrm{H} \alpha$ observations. A complete interpretation of these data is difficult because $\mathrm{H} \alpha$ is a chromospheric line, formed under conditions of non-LTE. The full understanding of the dynamic $\mathrm{H} \alpha$ structures would require a different interpretational method outside the scope of this thesis work. The magnetic properties of PFe and their relation with the global field are described in Sect. 5.2 Finally, Sect. 5.3 takes into account the dynamics of the plasma related with $\mathrm{PFe}$.

Firstly, a way to distinguish and differentiate PFe from other features in the observed FOVs must be developed. The main properties of PFe - i.e., their outstanding brightness above most background intensities, their strong magnetic field and their small size - are used to create a combination of three thresholds that select only the PFe in a FOV. For a structure to be considered a PF, its intensity must be $I_{\mathrm{bb}, \mathrm{PF}} \geq 1.1 \times \bar{I}_{\mathrm{bb}}$, where $\bar{I}_{\mathrm{bb}}$ is the average broadband intensity in the neighbourhood of the facula. As second threshold, for magnetic field strength obtained from the COG method, it is required that $\left|B_{\text {eff,PF }}\right| \geq 60 \mathrm{G}$. This value is approximately three times the noise of the magnetograms. Finally, a threshold in size was also applied in the sense that to be considered a PF, the feature must have

\footnotetext{
* Part of this Chapter is an extended version of Blanco et al. (2007).
} 
a minimum area of $2 \times 10^{4} \mathrm{~km}^{2}$, which corresponds to three contiguous pixels on the detector.

The above values are the ones chosen when dealing with FPI data. For TIP II observations, the thresholds in intensity and field strength are $I_{\mathrm{PF}} \geq 1.02 \times \bar{I}_{\mathrm{bb}}$ and $\left|B_{\mathrm{eff}, \mathrm{PF}}\right| \geq 18 \mathrm{G}$ (again, approximately three times the noise), respectively. The threshold in size is taken the same as for the FPI case. The threshold in intensity must be lowered for two reasons: a) Intensity enhancements due to temperature enhancements are much lower in the infrared at $1.56 \mu$ than at $6173 \AA$, a general property of Planck functions. b) The spatial resolution strongly influences the contrast, as described in Sect. 5.1.2 below, and the spatial resolution of TIP II observations is much lower than that of the FPI observations.

The combination of the three thresholds at the same time is necessary since, as noted when referring to Fig. 4.6 there exist some bright features with no magnetic field associated and vice versa. The magnetic flux stored in non-bright features is analysed in Sect. 5.2.3. The size threshold impedes some spurious signal - which are not bigger than one pixel, two contiguous at most - from entering the analyses.

This method of identifying PFe will be applied throughout all the different analyses that will be performed in this study.

However, the method is not completely reliable at all latitudes since some $\mathrm{PFe}$ are below the selection thresholds and thus would escape detection. Towards the disc centre $\mathrm{PFe}$ are not much brighter than their surroundings. Their contrast decreases and becomes similar to the contrast of bright granules. This is clearly seen below, in Fig. 5.4 Therefore, for FOVs observed at latitudes near the lower latitude boundary of PFe appearance, the intensity threshold was lowered in order not to create a bias in the PFe selection. Yet, the magnetic field threshold was kept at the same level. Both choices on intensity and magnetic field thresholds will be discussed in Sect. 5.1.2 and Sect.5.2.2 respectively.

Along with this difficulty at the lower boundary, it is to be noted that magnetic field signatures do not exactly coincide with the facular brightenings. The polarimetric signal appears shifted towards the disc centre with respect to the continuum intensity. The amount of the displacement increases towards the limb. Thus, closer to the limb, this shift can generate a bias in the facular identification. However, as can be seen in Fig. 5.1 the amount of the displacement even for the FOVs closest to the limb is not high enough to exclude a PF from being counted with the chosen thresholds.

Furthermore, before entering into the separate analyses and discussion of facular properties, an important result must be pointed out. Some earlier studies (e.g. Okunev \& Kneer 2004) stated that PFe possess unipolar magnetic fields with the same polarity as the global magnetic field. However, during the present work, many PFe of both polarities have been found near both solar poles, although a preponderance of PFe having the same polarity as the global field is observed (see Tables 5.2 and 5.3 and comments there). Also it has been observed that PFe from both polarities appear not as bipolar combinations, but as isolated magnetic patches.

In several of the following presentations of the results, PFe are separated according to their polarity as well as to the solar pole where they were observed. 

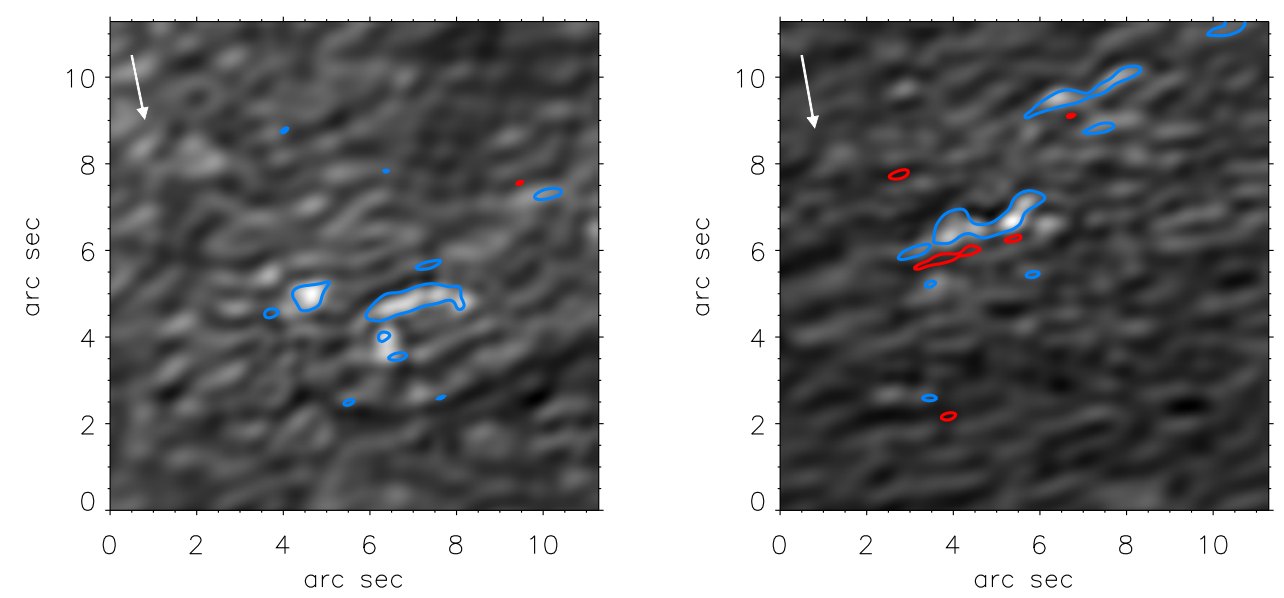

Figure 5.1: Examples of PFe at $\mu=0.34$ (left) and $\mu=0.20$ (right), sections of speckle reconstructed images overlaid with contours of magnetic field calculated from the COG method. Blue and red contours correspond to $-75 \mathrm{G}$ and $75 \mathrm{G}$, respectively. Arrows point towards limb.

\subsection{Photometric analysis}

The first analyses to be carried out correspond to the basic visual properties of PFe, i.e., brightness, number and size, since subsequent work is based on some of these properties. Except for Sect. 5.1.3, where intensity images from different positions along the $\mathrm{H} \alpha$ line profile are studied to follow the penetration of PFe into the chromosphere, the results presented here are obtained from the plain broadband and continuum images.

\subsubsection{Number and size density}

In order to understand deeper the relation of PFe with global solar phenomena, the first necessary study deals with the occurrence of PFe in the whole polar cap areas as well as their typical sizes. While PFe may be small and do not have such strong magnetic fields as sunspots, they could be polar phenomena of high relevance for the global magnetic field if they appear in sufficiently large numbers, given that their maximum occurrence takes place during sunspot minimum, i.e. when the solar magnetic field is predominantly poloidal. The PFe number and size would also be a constraint for simulations of magnetic flux transport from equator towards the poles and for the emergence of magnetic patches near the poles.

Not only is the number of PFe important for the polar magnetism but also for the solar wind (see Sect. 1.2). PFe could be sources of material feeding the fast solar wind out of the polar coronal holes, considering the coincidence in time and latitudinal extension of $\mathrm{PFe}$ appearance and polar coronal holes. But for PFe being the photospheric source of the fast solar wind, a sufficient amount of PFe is needed to match the material flux from the wind observed at Earth's distance as well as the magnetic flux contained in the wind.

In order to check whether there is some trend in the number of PFe observed with respect to the heliocentric angle in any of the two instruments, counts of $\mathrm{PFe}$ are grouped into intervals of $\Delta \mu=0.05$ without distinction between magnetic polarity or solar pole. 
The resulting facular numbers are given in Table 5.1 Some of the $\mu$ intervals show no PFe counts because there were no FOVs observed at that heliocentric angles.

Table 5.1: Number of observed PFe in intervals of $\Delta \mu=0.05$ together with the number of observed FOVs at those intervals in brackets, for both instruments.

\begin{tabular}{ccc}
\hline$\mu$ interval & FPI counts (FOVs) & TIP counts (FOVs) \\
\hline $0.20-0.25$ & $16(2)$ & - \\
\hline $0.25-0.30$ & - & - \\
\hline $0.30-0.35$ & $12(2)$ & $14(2)$ \\
\hline $0.35-0.40$ & $14(3)$ & $57(8)$ \\
\hline $0.40-0.45$ & $43(5)$ & $42(6)$ \\
\hline $0.45-0.50$ & $36(2)$ & $32(3)$ \\
\hline $0.50-0.55$ & $74(2)$ & $32(3)$ \\
\hline $0.55-0.60$ & - & - \\
\hline$>0.60$ & $62(3)$ & - \\
\hline
\end{tabular}

As seen from Table 5.1 both FPI and TIP II results show a sudden decrease towards the limb in the number of PFe at $\mu \approx 0.4$. Yet, according to the analyses in Sect. 5.1.2 and Sect. 5.2.2 no bias is introduced due to the intensity or magnetic field thresholds in the selection of PFe.

A possible cause of the descent in the number of PFe is of geometrical origin. Due to the increasing heliocentric angle, some PFe may not be visible as separate features but as forming part of a larger brightness structure because of a projection effect in three dimensions. Granules and embedded faculae are three-dimensional structures such that the interfacular areas become hidden when observing at high inclination angles with respect to the vertical. Limited spatial resolution then lets separated faculae appear as one structure.

To take into account the role of PFe in the global solar magnetism, an extrapolation from the limited surface covered with the observations to the whole polar cap area is necessary. The surface of a polar cap is

$$
S_{\mathrm{PC}}=\int_{60^{\circ}}^{90^{\circ}} 2 \pi R_{\odot}^{2} \cos \psi d \psi \approx 4.1 \times 10^{11} \mathrm{~km}^{2},
$$

with $R_{\odot}$ the solar radius and the integral performed between the extremal latitudes of PFe appearance during sunspot minimum. This will be the area considered for the extrapolation of the observations.

Due to the high heliocentric angles at which the observations were performed, and to the large FOVs observable with both instruments, the foreshortening effect - because of the planar projection of a spherical surface - is of relevance, changing also across the FOVs under observation. In order to take this into account, the FOVs were divided into sectors parallel to the limb. $\mu$ is different for each sector, thus also the correction factor $1 / \mu$. Each of the sectors was corrected according to its position, as shown in Fig. 5.2.

Once the PFe are selected from each FOV as described in the introduction to this section, they are segregated into north pole and south pole depending on where the PFe 
were observed. They are also separated according to their magnetic polarity. The results of the extrapolation are presented in Tables 5.2 and 5.3 for FPI and TIP II observations, respectively.

Table 5.2: Extrapolated number counts and extrapolated areas of PFe from FPI observations.

\begin{tabular}{ccc}
\hline \hline & total numbers & total areas $\left[10^{8} \mathrm{~km}^{2}\right]$ \\
\hline north pole, total & 4120 & 7.65 \\
magn. positive & 620 & 0.65 \\
magn. negative & 3500 & 7.0 \\
\hline south pole, total & 1250 & 3.44 \\
magn. positive & 720 & 2.8 \\
magn. negative & 530 & 0.64 \\
\hline
\end{tabular}

Table 5.3: Same as Table 5.2 for TIP II observations

\begin{tabular}{ccc}
\hline \hline & total numbers & total areas $\left[10^{8} \mathrm{~km}^{2}\right]$ \\
\hline north pole, total & 4365 & 24.7 \\
magn. positive & 1440 & 4.5 \\
magn. negative & 2925 & 20.2 \\
\hline south pole, total & 2720 & 18.8 \\
magn. positive & 1630 & 13.9 \\
magn. negative & 1090 & 4.9 \\
\hline
\end{tabular}

First of all, one notices that PFe with magnetic polarity opposite to the global magnetic field are not a rare phenomenon but in fact, they do appear often. Up to $20-30 \%$ of the total amount of PFe found in these observations presented opposite polarity.

An important asymmetry is observed between north and south poles in the total number of PFe as well as in the ratio of polarities, especially in FPI observations. With regard to these latter, there were almost four times more PFe near the north pole than near the south pole. With respect to TIP II data, nearly two times more PFe were observed close to the north pole than around the south pole. The ratio is much lower, i.e. closer to one, for opposite polarity PFe (magnetically positive at the north pole and magnetically negative at the south pole) in both cases.

This effect could be due to the different number of observed FOVs near the north and south poles. In the case of FPI observations, the total observed area near the north pole was almost three times larger than that near the south pole. This fact possibly introduced a bias during the extrapolation due to low number counts. However, for TIP II observations the total observed areas were very similar for both poles and the asymmetry is still present in these data.

The reason for the lower observational coverage of the southern polar cap in the case of FPI observations is a consequence of the inclination of the solar axis with respect to the 
ecliptic pole. During the FPI observational campaigns the northern polar cap was better visible and observations could be carried out to lower heliocentric angles $\theta$ than near the south pole. For TIP II data, the solar equator was seen closer to edge-on, so none of the poles was better observable than the other.

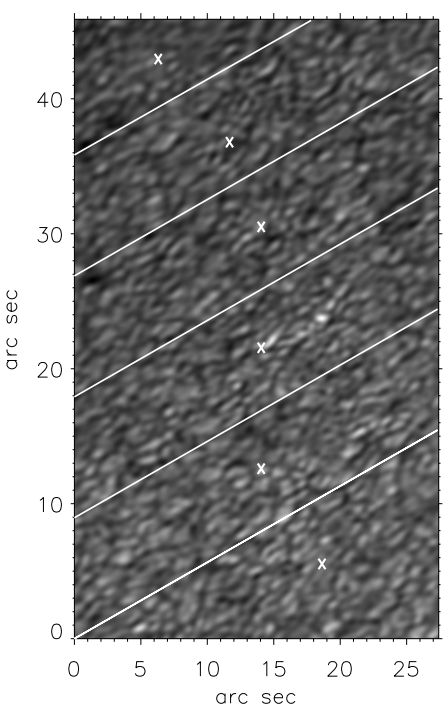

Figure 5.2: Example of correction for foreshortening. White lines are parallel to the limb. Crosses mark the centre of each sector.

Benevolenskaya (2004) also found asymmetries (in total magnetic fluxes) between both poles when analysing SoHO/MDI magnetograms in a more restricted area than that studied here, between heliographic latitudes $78^{\circ} \leq|\psi| \leq 88^{\circ}$, but with no observational coverage difference. This, combined with the present TIP II results, points more in the direction of an intrinsic asymmetry between north pole and south pole than to an observational bias. Further observations of both poles at the same epoch are needed to confirm this difference.

Figure 5.3 depicts the distribution of areas of $\mathrm{PFe}$, separately for both poles and both magnetic polarities. The areas are corrected for the foreshortening effect, by dividing them by $\mu$. The two left panels show the distribution of areas for PFe on the northern cap, separated into negative and positive polarities in the upper and lower panels, respectively. The right panels show the same distributions for the south pole. The results of observations from both instruments, FPI and TIP II, appear as red and black lines, respectively.
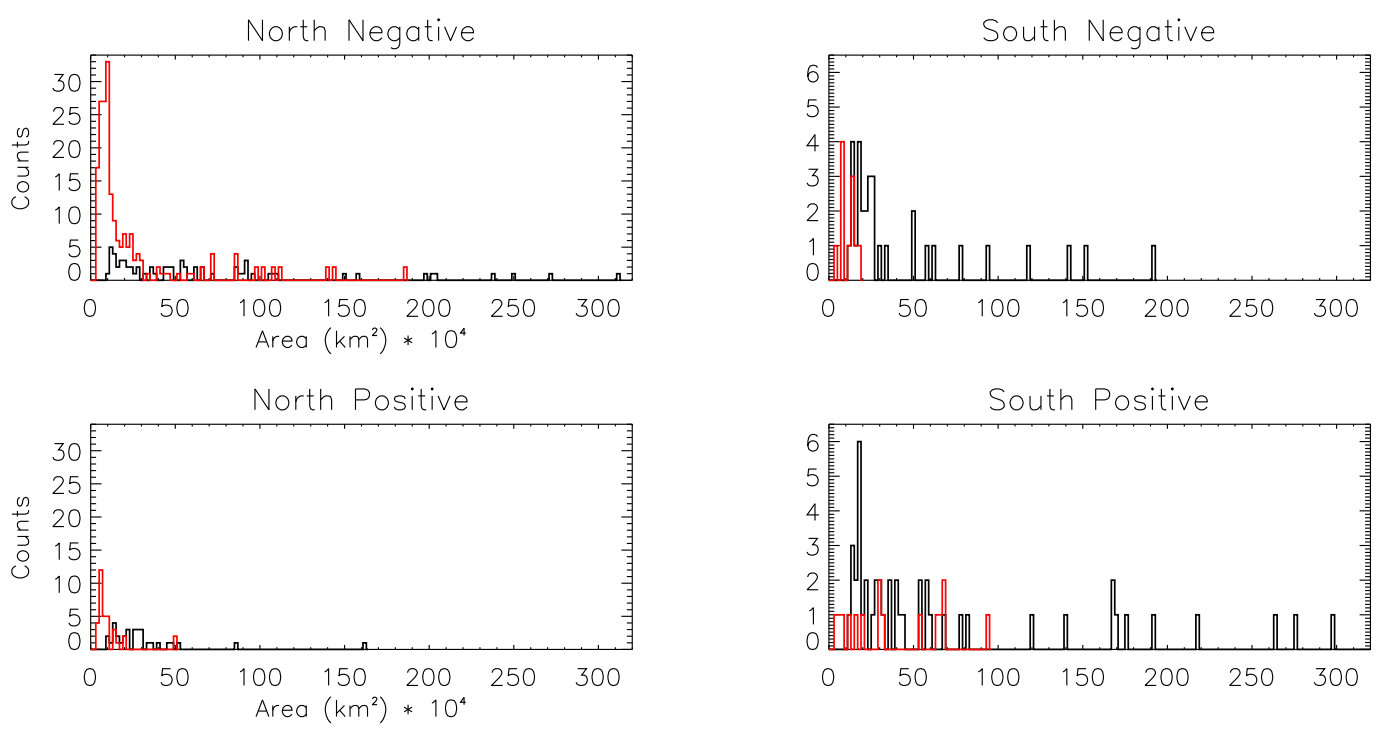

Figure 5.3: Distribution of PFe areas. Two left panels correspond to PFe at the northern polar cap, with negative (upper panel) and positive (lower) polarities. Right panels are from the southern polar cap. Red lines depict the area distributions from FPI data, black lines from TIP II data.

From Fig. 5.3, the distribution of sizes obtained from TIP II data peaks always at larger 
areas than that from FPI data. This fact also arises from the comparison of total extrapolated areas in Tables 5.2 and 5.3. This is the result of the lower spatial resolution of TIP II observations which impedes to discriminate smaller PF components in conglomerates. In both cases, FPI and TIP II, PFe of the same polarity as the global field are larger on average than those of opposite polarity. Since the global magnetic field is poloidal (to a good approximation) at this epoch, the flux in PFe of the same polarity must be larger to contribute actively to the total magnetic flux of the corresponding pole.

The mean size of all observed $\mathrm{PFe}$ - both poles, both polarities - is approximately of $25 \cdot 10^{4} \mathrm{~km}^{2}$. Supposing a circular shape for PFe, the mean area implies a radius of approximately $270 \mathrm{~km} \approx 0.33^{\prime \prime}$. This underlines, the importance of very high spatial resolution to distinguish the small $\mathrm{PFe}$ as separate features.

\subsubsection{Centre-to-limb variation of contrast}

With respect to the local quiet Sun, magnetic field features present enhanced or decreased intensity, depending on their size. Strong magnetic fields inhibit the convection in the subphotospheric layers and thus, the energy input and the temperature are reduced. However, PFe appear brighter than the surrounding atmosphere. Inside the tube, the density is lower because of evacuation of the tube caused by the magnetic pressure - the external and internal pressures of the tube must balance, $p_{\text {gas }}^{\text {ext }}=p_{\text {gas }}^{\text {int }}+p_{\text {mag }}$. When observing at large heliocentric angles, one obtains more radiation from the walls of a vertical magnetic tube than near disc centre and due to the evacuation, deeper, hotter layers of the ambient atmosphere than the one observed outside the tube, can be seen (hot wall model, see Spruit 1976). Studying the contrast of PFe as well as its variation towards the limb can provide valuable information about the internal thermal structure of PFe.

Furthermore, the change from sunspots to PFe during the activity cycle contributes to the variation of the solar irradiance (Solanki \& Fligge 2002) and constraining the contrast of $\mathrm{PFe}$, and of faculae in general, would help in modelling the variance.

During the observational campaigns, $\mathrm{PFe}$ were observed at latitudes from $\mu \approx 0.6$ to $\mu \approx 0.2$, i.e. nearly their whole region of appearance during sunspot minimum, except for the very limb. This high coverage together with the high number of PFe found, allows a statistical analysis of the contrast of $\mathrm{PFe}$ and its variation with heliocentric angle.

The contrast $C$ is defined as

$$
C=\frac{\left(I_{\mathrm{bb}, \max }-\bar{I}_{\mathrm{bb}, \mathrm{FOV}}\right)}{\bar{I}_{\mathrm{bb}, \mathrm{FOV}}},
$$

where $I_{\mathrm{bb}, \max }$ denotes the maximum broadband intensity in each facula and $\bar{I}_{\mathrm{bb}, \mathrm{FOV}}$ is the average broadband intensity of the whole FOV. A normalisation to a second order surface was applied to the images before measuring the contrasts, to remove the centre-to-limb variation (CLV) of the broadband intensity within each FOV.

The determination of the contrast from Eq. 5.2 was performed with different averages of $\bar{I}_{\mathrm{bb}, \mathrm{FOV}}$. The averages were calculated including the PFe present in the FOV and excluding them. Averaging only the local surrounding intensity was also considered. The differences from the three methods turned out to be negligible.

In Fig. 5.4 $\mathrm{a}$ ) the mean contrast of PFe from each FOV versus the heliocentric angle is 
presented. The standard deviations of PF contrast within each FOV are depicted as error bars. Dashed lines correspond to linear fits to the contrast values as a tentative indication of the CLV of the contrast. Before performing the linear fits, the measurements have been split into two groups according to their heliocentric angle, with $\mu \geq 0.42$ and $\mu \leq 0.42$. The reason for this separation is the apparent difference in the steepness of variation of PF contrast. Towards the disc centre, the contrast of PFe decreases whereas towards the limb it stays approximately constant or increases slightly to $C \approx 0.4$. This not-decreasing behaviour and the amplitude of the contrast coincide with the CLV obtained with the same speckle reconstruction as applied here by Hirzberger \& Wiehr (2005).
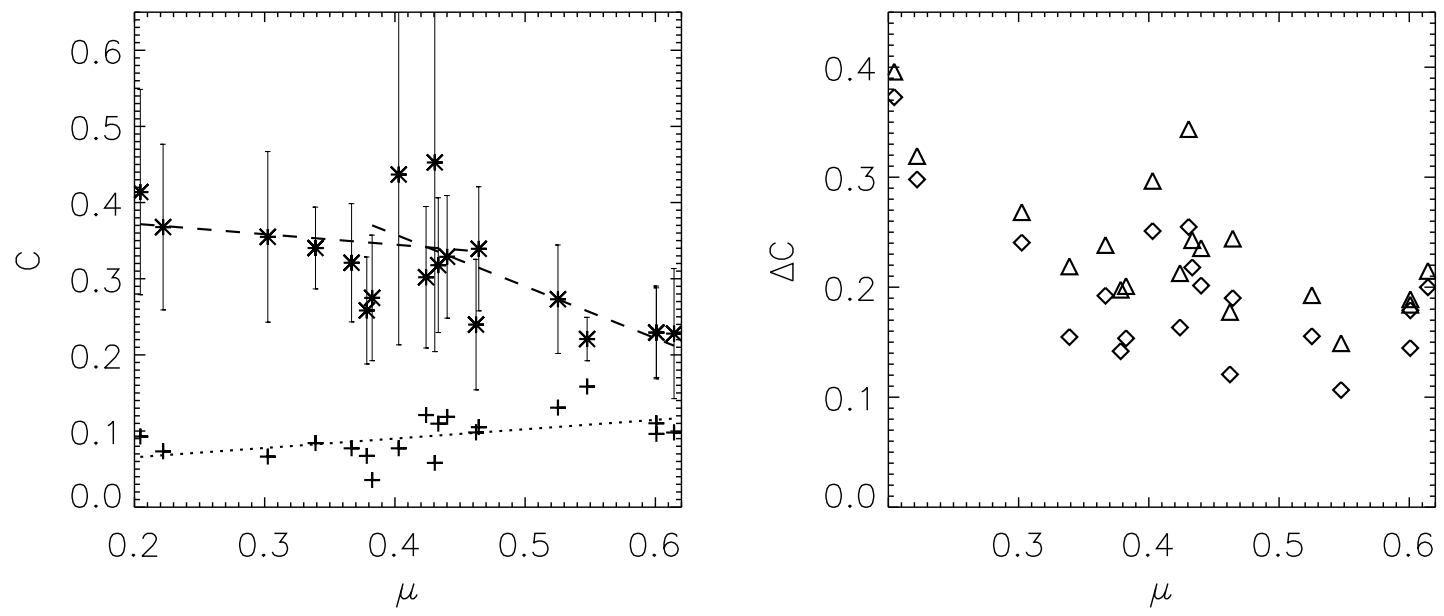

Figure 5.4: a) Contrast $C$ of PFe and of granules vs. $\mu$. The asterisks give the mean contrast of PFe within one FOV and the error bars represent the standard deviation of facular contrast within the same FOV. The dashed straight lines are linear fits to the data split into two sets, delineating tentatively the variation of facular contrast towards the limb. The crosses show twice the rms contrast of granules in the FOVs with no (or little) magnetic flux, $2 \times C_{\mathrm{rms} \text {,gran }}$ (see text). The dotted straight line is a least square fit to the granular contrast. b) Difference of contrast for different resolutions vs. $\mu$. Triangles correspond to the difference of contrast $C$ for PFe between the reconstructed image and the average image of the burst, and diamonds to the difference between the reconstructed image and the "best" speckle image of the burst.

For comparison with the PFe contrast, the contrast of the brightest granules in the quiet Sun is also shown in Fig. 5.4 a). To estimate this contrast, twice the rms contrast of granules was used, $2 \times C_{r m s, \text { gran }}=2 \times \sigma$. (For a pure two-dimensional sinusoidal intensity distribution, one has $2 \times \sigma=A$, with $A$ the amplitude. For two-dimensional Gaussian noise, the threshold $\Delta I / \bar{I} \geq 2 \times \sigma$ gives the brightest intensity values.) The dotted line represents a linear fit to the granular contrast just as an indication of the trend, with no scientific interpretation. Fluctuations around this regression line are due to the low number of granules with contrast higher than $2 \times \sigma$. Towards the disc centre, both the contrast of PFe and that of the bright granules become similar. Hence, near the disc centre it is difficult to distinguish between PFe and granules from a broadband or continuum intensity analysis alone.

Note also that, although in other properties (e.g., Sect. 5.1.1 Sect. 5.2 Sect. 5.3) an asymmetry in the behaviour between PFe from north and south poles has been observed, no difference is noticeable regarding their contrast.

A wide range of values has been published in the literature concerning the facular and 
bright point contrast and their CLV (Lawrence \& Chapman 1988; Auffret \& Muller 1991; Ortiz et al. 2002; Okunev 2004). One of the main reasons for the different values seems to be the spatial resolution of the data - with higher spatial resolution leading to higher contrast. Domingo et al. (2005) found also, from MDI observations, that the trend of the CLV changes with the spatial resolution. For high-resolution data, the contrast towards limb was always increasing whereas for low-resolution data the contrast presented a maximum around $\mu=0.4$ and decreases towards limb. In Fig. 5.4b), the difference between the contrast of PFe in the "best" speckle image of the burst and in the reconstructed image is presented, and the difference between the contrast of PFe in the average image and in the reconstructed one. Obviously, the PF contrasts obtained from the reconstructed images are always higher than those from the average and the best images. For $\mu \geq 0.4$, this difference stays approximately constant towards the disc centre at $\Delta C \approx 0.2$, whereas towards the limb there is an increase of the difference. This result is similar to what Domingo et al. (2005) found. When comparing semiempirical atmospheric models with observations from the Precision Solar Photometric Telescope at Mauna Loa, Penza et al. (2004) find that network features show a decrease in contrast near the extreme limb. They suggest that this results from the instrumental profile (i.e. limited spatial resolution) causing the measurement of the thin network elements to be "polluted" by an increasing quiet Sun area dependent on the heliocentric angle. The facular contrast is not so much affected because facular structures are more extended than network elements.

However, the present results differ from the suggestion made by Domingo et al. (2005) that in regions of isolated flux elements the CLV would decline.

\subsubsection{H $\alpha$ analysis}

The height of formation of the $\mathrm{H} \alpha$ line, up to the chromosphere (at $1200-1700 \mathrm{~km}$, Vernazza et al. 1981) at the line core, and down to 100-300 km in the wings, allows to study the behaviour of PFe through different atmospheric layers. Also, a photometric analysis of $\mathrm{H} \alpha$ is useful as proxy for magnetic elements.

Leenaarts et al. (2006) analysed observations from the Dutch Open Telescope in different wavelengths in order to refine intensity proxies for small-scale magnetic elements. On the basis of G-band bright points - widely used indicators for small intergranular magnetic elements -, they performed a comparison with $\mathrm{Ca}$ II $\mathrm{H} \& \mathrm{~K}$ lines and with the blue wing of $\mathrm{H} \alpha$ at $-0.8 \AA$ off line centre. From their analyses, they conclude that the blue wing of $\mathrm{H} \alpha$, although giving less sharp images than the G-band, is a better diagnostic tool to track intermittent magnetic elements.

$\mathrm{H} \alpha$ data obtained for the present work have the advantage of having been observed at 23 different positions across the line profile. Also, the fact that these observations are performed quasi-simultaneously in a magnetically sensitive line and in $\mathrm{H} \alpha$ permits the direct comparison between magnetic field and $\mathrm{H} \alpha$ brightness. This allows to study the relation of magnetic fields with $\mathrm{H} \alpha$ structures in a wavelength range of $2 \AA$ across the profile. Figure 5.5 shows a subfield of a speckle reconstructed image from FPI data at $\mu=0.46$, at 21 different wavelength positions in the $\mathrm{H} \alpha$ line. Overlaid in each frame are contours of magnetic field strength at $75 \mathrm{G}$ (blue) and $-75 \mathrm{G}$ (red), measured from the COG, and contours of $\mathrm{H} \alpha$ (green) with an intensity $14 \%$ higher than the mean at the corresponding wavelength position. 
$-1006.70 \mathrm{~mA}$

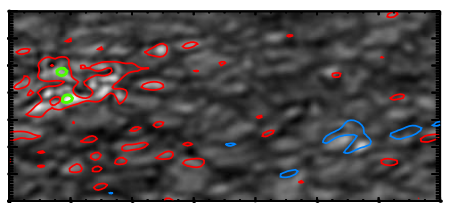

$-704.690 \mathrm{~mA}$

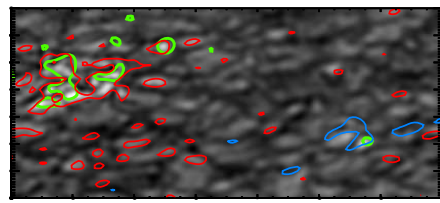

$-402.680 \mathrm{~mA}$

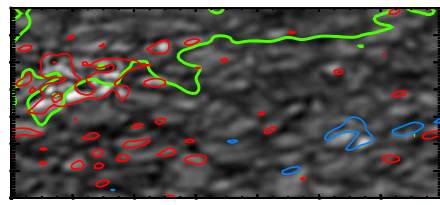

$-100.670 \mathrm{~mA}$

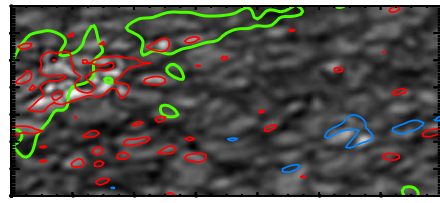

$201.340 \mathrm{~mA}$

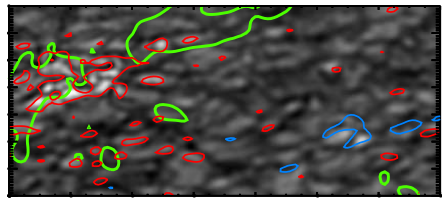

$503.350 \mathrm{~mA}$

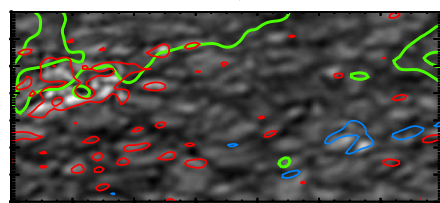

$805.360 \mathrm{~mA}$

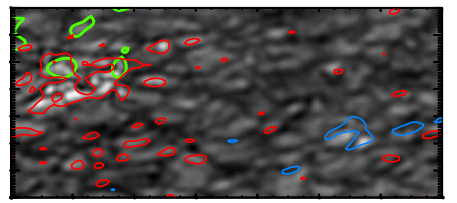

$-906.030 \mathrm{~mA}$

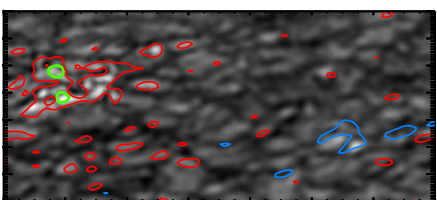

$-604.020 \mathrm{~mA}$

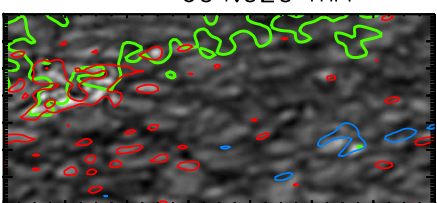

$-302.010 \mathrm{~mA}$

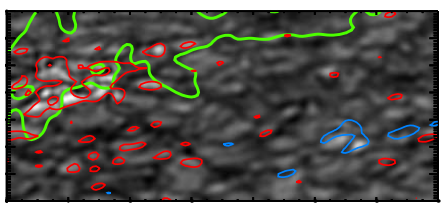

$0.00000 \mathrm{~mA}$

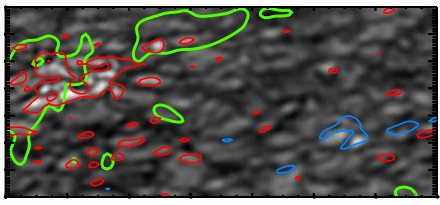

$302.010 \mathrm{~mA}$

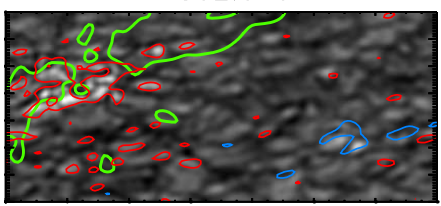

$604.020 \mathrm{~mA}$

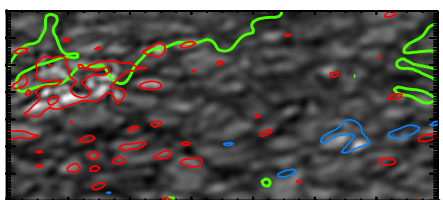

$906.030 \mathrm{~mA}$

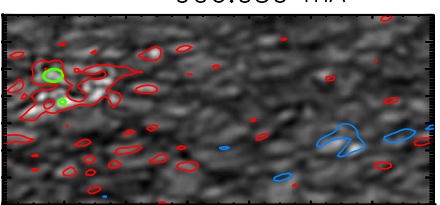

$-805.360 \mathrm{~mA}$

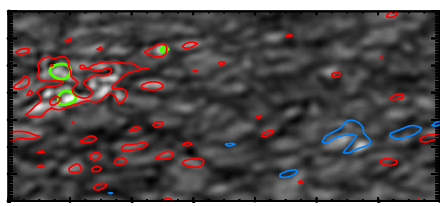

$-503.350 \mathrm{~mA}$

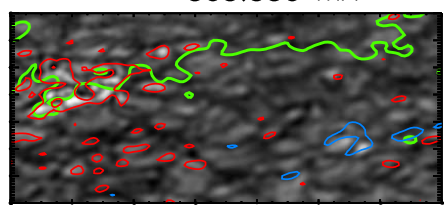

$-201.340 \mathrm{~mA}$

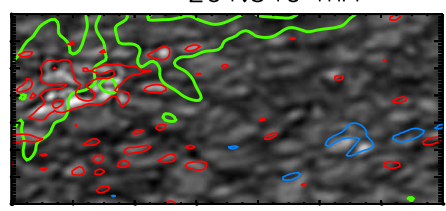

$100.670 \mathrm{~mA}$

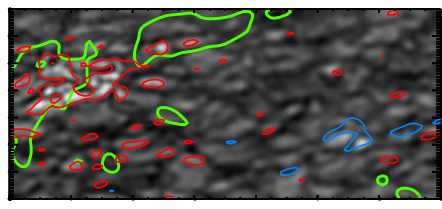

$402.680 \mathrm{~mA}$

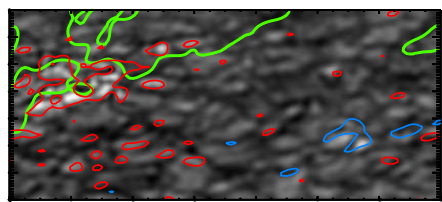

$704.690 \mathrm{~mA}$

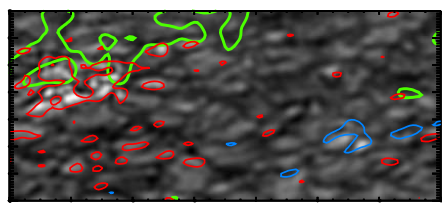

$1006.70 \mathrm{~mA}$

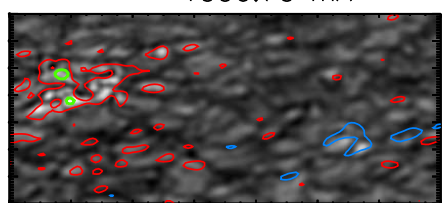

Figure 5.5: Section of speckle reconstructed broadband image overlaid with contours of magnetic field (red at -75 Gauss, blue at 75 Gauss) and $\mathrm{H} \alpha$ brightenings (green at $14 \%$ brighter than mean intensity at each wavelength position). The numbers in $\mathrm{m} \AA$ show the position with respect to the centre of the H $\alpha$ line. The arrow in the lower left image points towards the limb.

From Fig. 5.5 it seems that the bright structures seen in $\mathrm{H} \alpha$ are expanding with height, with its centres in the magnetic elements, when advancing in wavelength from the blue wing to the line core. Yet, near the centre of the line, i.e. in the chromosphere proper, the intensity pattern changes strongly from the images in the wings. On the other side of the line, towards the continuum in the red, the intensity pattern becomes again similar to that of the blue wing, although not identical. The comparison of images in the blue and 
red wings of $\mathrm{H} \alpha$, as proxies for the magnetic elements, shows that the blue wing depicts much more precisely the concentrations of magnetic field than the red wing.

In the example given in Fig. 5.5 the best wavelength position for using $\mathrm{H} \alpha$ as a magnetic field proxy, is at $-0.7 \AA$ off line centre, similar as the $-0.8 \AA$ intensities analysed by Leenaarts et al. (2006). As visible also at e.g. $-0.7 \AA$, the polarity of the magnetic field is of no relevance, $\mathrm{H} \alpha$ traces both polarities. At further wavelength positions the intensity contours of the positive polarity disappear because the intensity pattern is masked by a nearby darker $\mathrm{H} \alpha$ structure.

Brightenings in the red wing near $\mathrm{H} \alpha$ line centre $(+0.2 \AA-+0.4 \AA)$ appear as elongated structures displaced from the magnetic elements. This is different in the blue wing where the brightenings include the magnetic elements.

Two further notes concerning the $\mathrm{H} \alpha$ analysis and results are appropriate: First, as with the intensity threshold for selecting PFe, the threshold used for $\mathrm{H} \alpha$ in Fig. 5.5 must be lowered when observing at lower latitudes, i.e. closer to the boundary of PFe appearance. Second, whereas $\mathrm{H} \alpha$ has proved to be a good proxy for PFe, it does not mark properly (if at all) magnetic elements without associated brightness in continuum. Whether this phenomenon is rare or common requires further observations.

\subsubsection{Temporal evolution}

During the April 2006 campaign, a long time series of a particlar FOV, lasting approximately 6 hours, was observed. Throughout the whole time series, the PF was recognizable although continuously changing, as seen in Fig. 5.6, where the time-series images have been co-align.
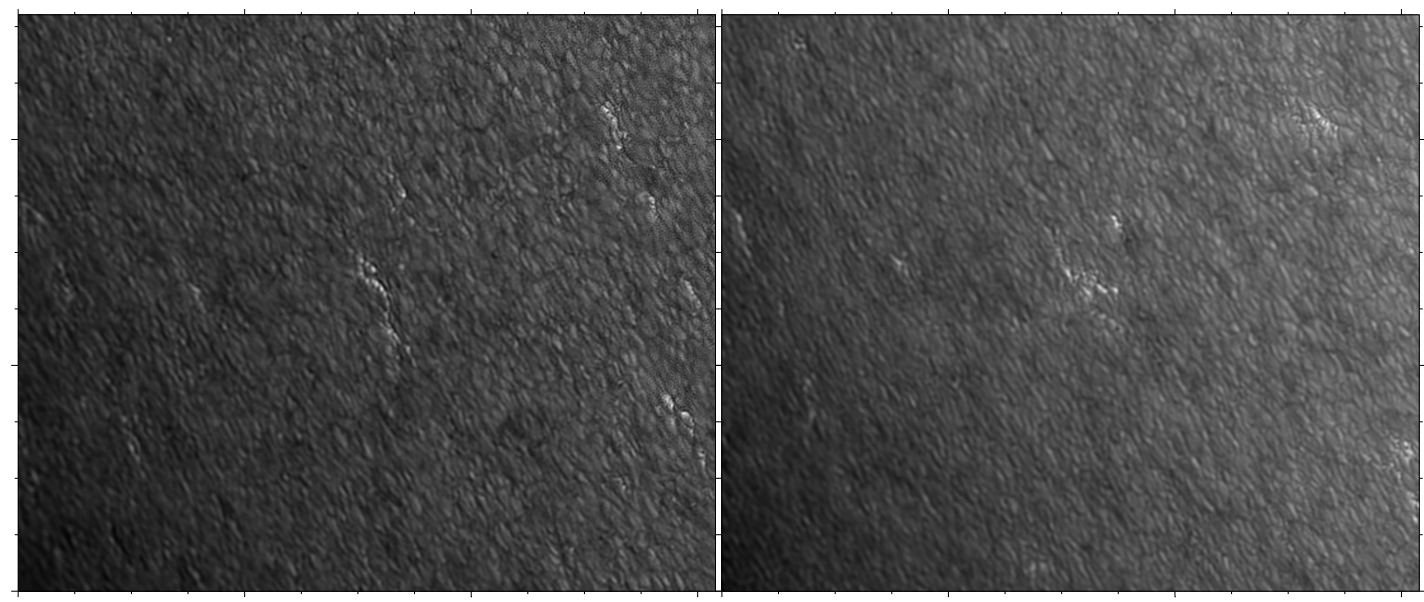

Figure 5.6: Speckle reconstructed images of the same FOV at $\mu=0.31$. Data are from 08:56 UT (left) and 14:13 UT (right). Tickmark distances correspond to $5^{\prime \prime}$.

In their study of the polar rotation of the Sun, Solonski \& Makarova (1992) analysed PFe with lifetimes longer than 8 hours. From their observations, they found that only $16 \%$ of the total number of PFe had lifetimes of that order.

Albeit with limited information from only one FOV, in the present observations apparently 
all of the PF structures remain for the whole time series with no or only little relative shifts across the FOV.

Thanks to the fast scanning of the new FPI, the evolution of PFe can be studied in time scales of approximately 10 seconds from the speckle reconstructed images, as presented in Fig. 5.7 However, the magnetic field measurements only have a cadence of approximately 40 seconds. In the present work only the intensity aspect of the long time series has been analysed.

Figure 5.7 shows a section of the time series data starting at 08:56 UT, which focuses on the central conglomerate shown in Fig. 5.6, The sequence in Fig. 5.7 covers almost four minutes, although a gap of approximately 90 seconds exists between the twelth and thirteenth images due to a failure of the AO.

Note at this closer look and at this time cadence, the many small scale activity that is taking place. From this analysis, the time evolution of PFe is in the order of ten seconds, shorter than thought before.

In Fig.5.7 two brightenings are marked with circles. The upper one shows an increase and subsequent decrease in intensity in a time interval of 2 min. The lower one also presents an intensity increase at the beginning. Afterwards, the intensity diminishes while nearby brightenings appear. In the last row, after the $90 \mathrm{~s}$ gap, there is no appreciable brightness in the lower circle.

For future PF studies, a fast time evolution measurement of PF magnetic field with high spatial resolution will be a primary option in order to analyse whether these increasing/decreasing brightenings are due to changes in magnetic field strengths or to lateral shifts of magnetic fields. Or whether the brightenings respond to a completely different phenomenon, such as convection in the ambient granulation (cf. De Pontieu et al. 2006).

\subsection{Magnetic field}

The study of PF magnetic fields is relevant for both the characteristic properties of PFe and the role of PFe for the global magnetic field. The fact that they possess a strong field together with their high number of appearance during sunspot minimum implies that $\mathrm{PFe}$ are important features for the polar magnetic flux. In this Section, both their internal magnetic field and its relation with the polar field are analysed. Also, the magnetic flux outside PFe, i.e. in structures with no associated brightening, is measured and compared with that contained in PFe and in the polar caps.

\subsubsection{Comparison of methods}

In Sect. 4.3 different methods to obtain the magnetic field were presented. Three of them measure the LOS component of the magnetic field, the COG method, the amplitudes from two-Gaussians fit and the least-squares calculation of Eq. 4.23. Also, a theoretical study from synthetic profiles of the four methods - including the SFR - was performed and the results were shown in Fig. 4.7. Here, another comparison between the three methods for the LOS component is presented in Fig. 5.8, from actual observations. The data are from the August 2005 campaign. 

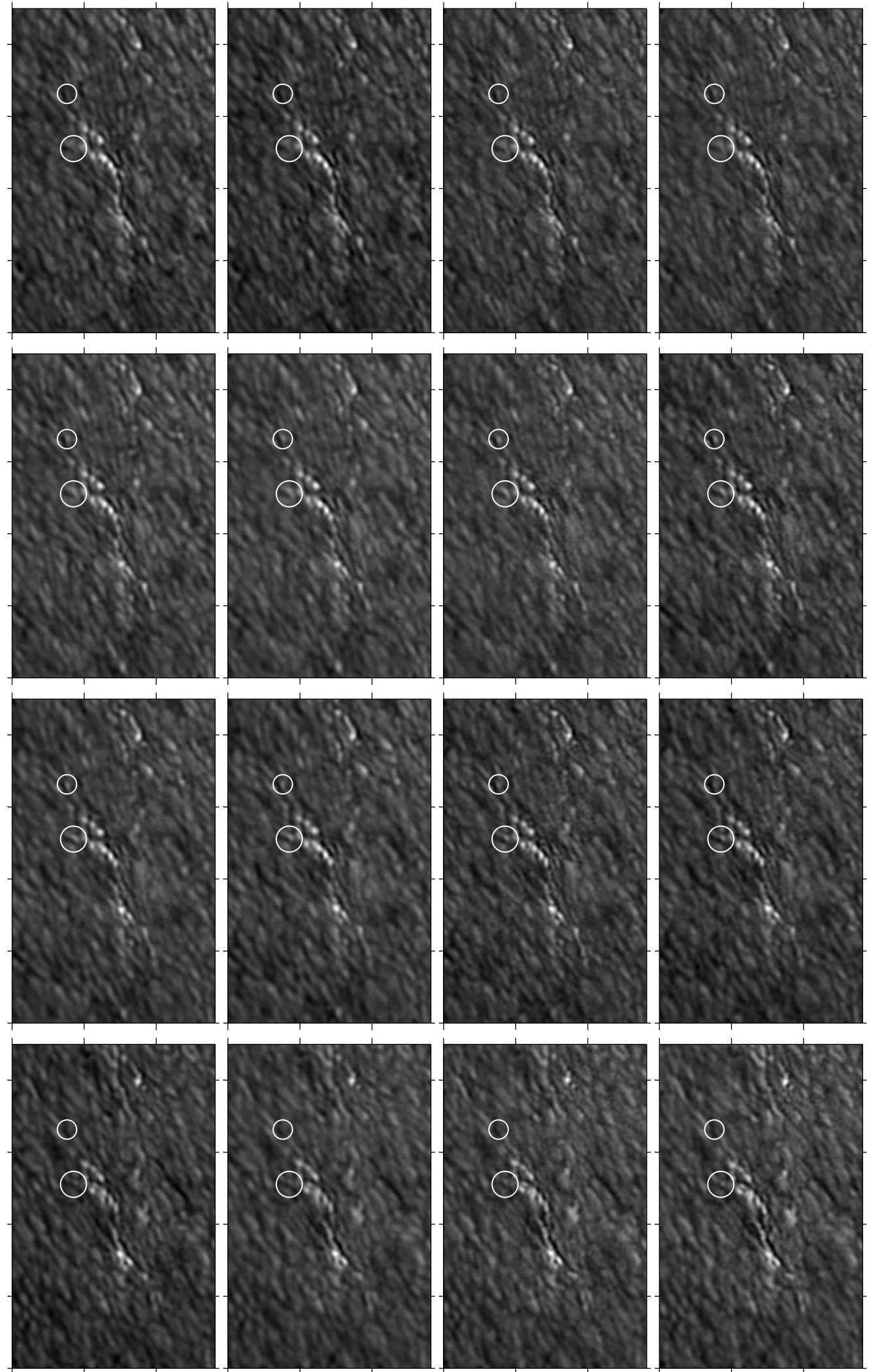

Figure 5.7: Section of speckle reconstructed images from the time series. The series goes left to right and top to bottom, with a cadence of $\approx 10 \mathrm{~s}$. Between the third and fourth row there is a gap of $\approx 1 \mathrm{~min}$. Circles mark appearing-disappearing PFe. Tickmark distances correspond to 5". 

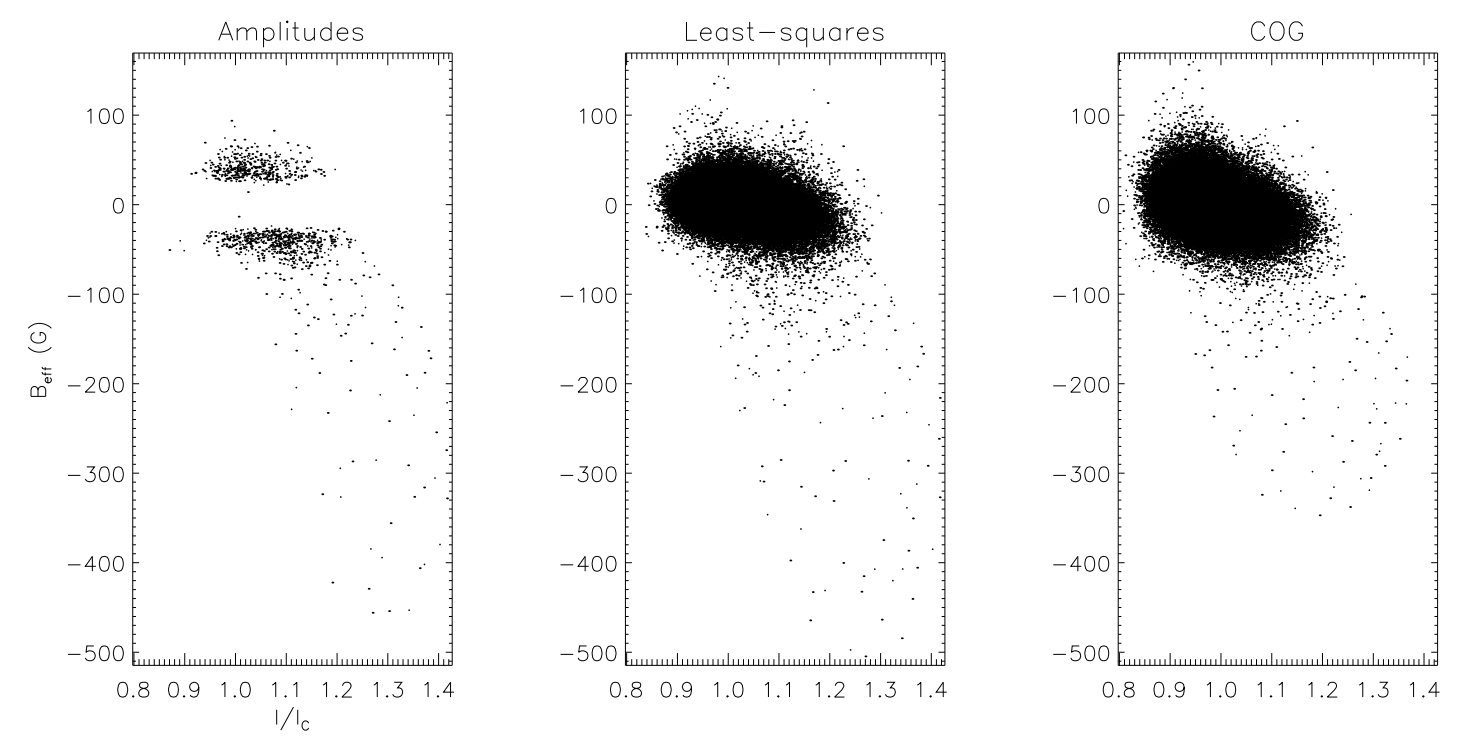

Figure 5.8: Comparison of the three methods applied for the determination of $B_{\mathrm{eff}}$ from a FOV at $\mu=0.5$.

The most noticeable fact is that the plot from the two-Gaussians fit (left panel in Fig. 5.8 has a lower limit of field detection. Also the areas where the magnetic field is not zero are less crowded than in the other two cases. Both these two facts are a consequence of the noise, thus limited capability of the fitting procedure, as mentioned in Sect. 4.3

The least-squares calculation and the COG methods give very similar results as was also noted in Sect.4.3. For mid field strengths the least-squares fit shows some overestimation while for low field strengths the COG method gives some overestimation, as in the synthetic profiles analysis. Thus, most of the studies involving $B_{\text {eff }}$ presented in this work are performed with values of $B$ from the COG method.

\subsubsection{Polar Faculae}

Here the different methods to measure magnetic fields will be applied to PFe selected as explained above. Section 5.2.2.1 deals with the results from the LOS component of the magnetic field. Section 5.2.2.2 analyses the results of the total magnetic field strength from the SFR, which are used in Sect. 5.2.2.3 to compare the flux from PFe with the flux from the polar caps determined earlier by other methods.

\subsubsection{Centre Of Gravity and Weak Field Approximation}

Figure 5.9 depicts the average effective field of each PF measured by means of the COG method, vs. the heliocentric angle for FPI (left) and TIP II (right) observations, respectively. No distinction with respect to the magnetic polarity or whether the PFe belong to the north or south pole is made.

The difference in the strength of the LOS component between the visual and infrared lines - around 4 times lower in the infrared - is puzzling. A possible explanation is the much lower spatial resolution in TIP II data compared with FPI data. This yields a higher influ- 

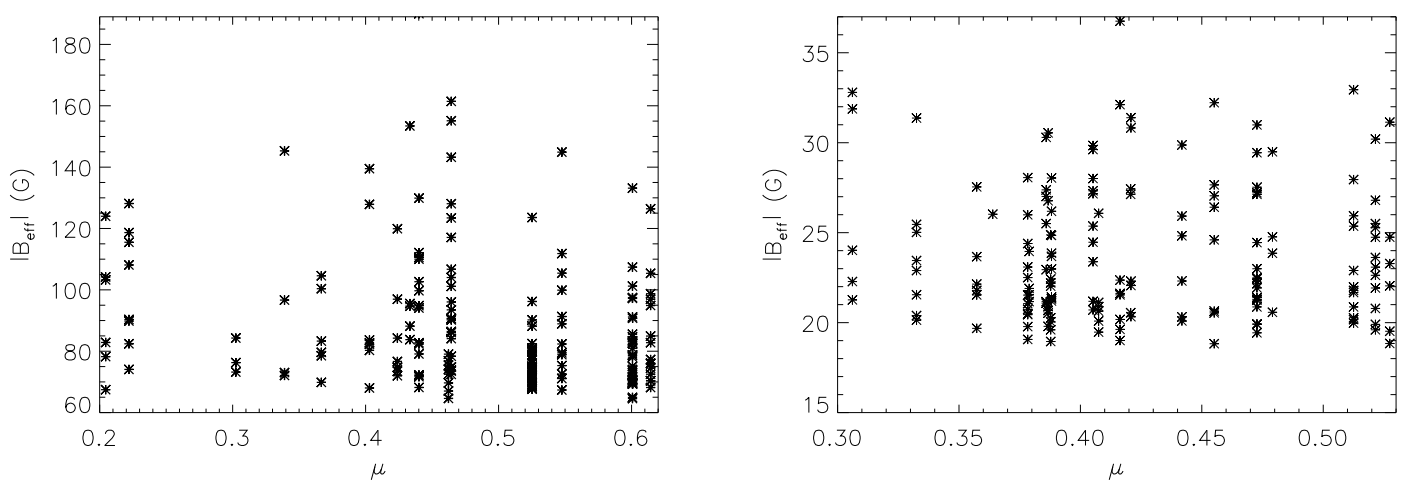

Figure 5.9: Variation towards the limb of the LOS component of magnetic field strength $\left|B_{\text {eff }}\right|$ of $\mathrm{PFe}$ measured with the COG method for FPI observations (left) and TIP II observations (right). The asterisks represent averages from each PF.

ence of profiles of unpolarised light in the resolution element, which causes the COG to measure lower fields than expected. Figure 5.10 shows a section of Stokes $I$ and $V$ spectrograms of one FOV from TIP II observations. The high separation of the Stokes $V$ lobes

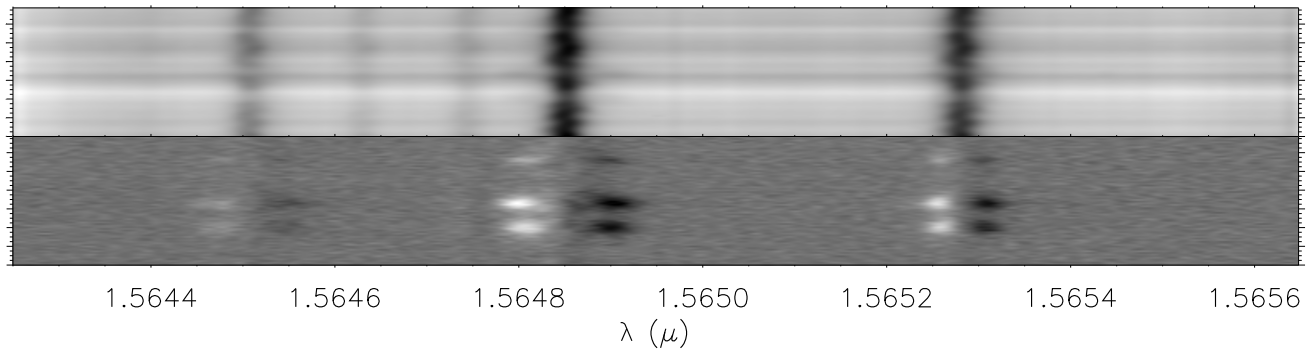

Figure 5.10: Section of Stokes I (upper) and $V$ (lower) spectrograms of a TIP II FOV at $\mu=0.39$. Each major tickmark corresponds to 2". Data from May 2007 campaign.

indicates a stronger magnetic field than the one measured with the COG. The strength of these fields is described in Sect. 5.2.2.2

Both sets of observations, FPI and TIP II, give the same results concerning the variation of $B_{\text {eff }}$ with the heliocentric angle. The measured LOS magnetic fields show no centre-to-limb variation.

Explanations, such as a bias caused by specific methods applied or an instrumental crosstalk effect, have to be discarded. As shown in Fig. 4.7 the COG method exhibits an almost linear behaviour in the measurement of the LOS component of the magnetic field for the whole range of input fields, it works reliably. On the other hand, as explained at the end of Sect. 4.3 previous works suggest that crosstalk would yield a weaker measured magnetic field strength than the actual one, thus producing a steeper decay of the field strength towards limb. Besides, TIP II data are free of crosstalk effects and, although 
in a narrower range of $\mu$ than FPI observations, still show no centre-to-limb variation. Thus, the choice of the $60 \mathrm{G}$ threshold (18 G for TIP II data), independent of heliocentric angle, does not introduce any bias in the detection of PFe.

For TIP II observations, where full-Stokes polarimetry could be performed, the transversal magnetic field strength has also been measured. Figure 5.11 shows the centre-tolimb variation of the transversal field similarly as in Fig. 5.9 with the LOS component, the asterisks denoting averages from each PF. As for the LOS component, no variation of

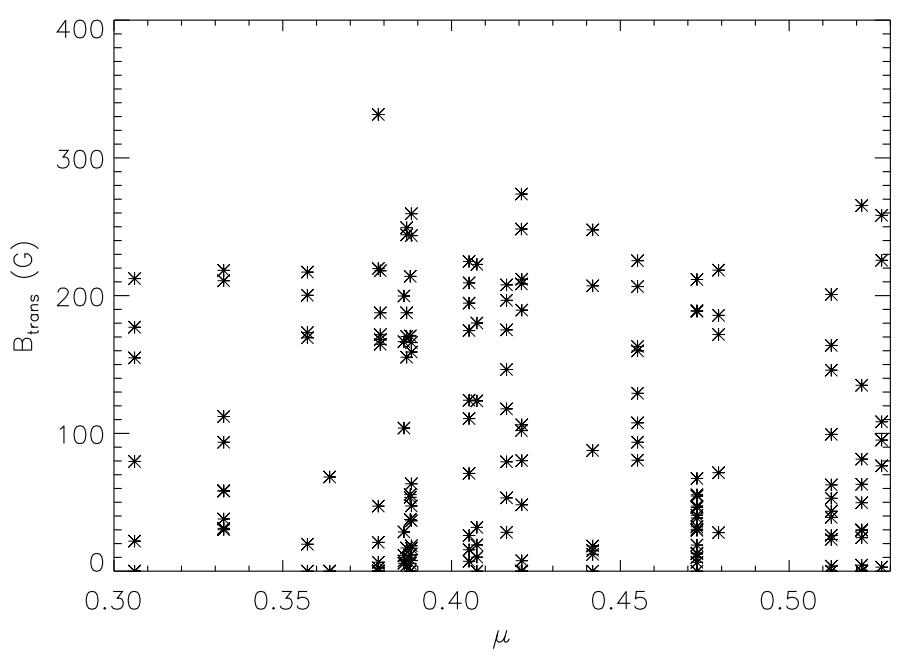

Figure 5.11: Variation towards the limb of the transversal magnetic field component in PFe obtained with a least-square fit to TIP II data in the WFA, Eqs.4.24 and 4.25 Asterisks represent averages from each PF.

the transversal magnetic field component towards the limb is noticeable. A comparison between the values in the right panel of Fig. 5.9 and in Fig. 5.11 shows that, from TIP II observations, the strength of the LOS component amounts only to approximately $20 \%$ of that of the transversal component on average. Following the possible explanation for the lower strengths of the LOS component of the field from TIP II data compared with the FPI data, the difference between LOS and transversal component here could come from the different methods used to derive the measurements, COG and Eqs. 4.24 and 4.25

These findings, including the absence of a noticeable variation towards the limb of the LOS and of the transversal magnetic field component, are difficult to understand. Further studies are definitely needed.

\subsubsection{Strong Field Regime}

In order to relate the magnetic flux from PFe to the global poloidal flux, two things are needed. First, the total area occupied by PFe, which was already calculated from the extrapolations and presented in Tables 5.2 and 5.3 for FPI and TIP II observations, respectively. Second, the total magnetic field strength inherent to PFe.

To obtain the total strength, as described in Sect. 4.3, the SFR is applied. The SFR resuls of the PF field strengths are given in the histograms in Fig. 5.12 Here, the values for the north and south solar poles are given by the solid and dashed lines, respectively. 

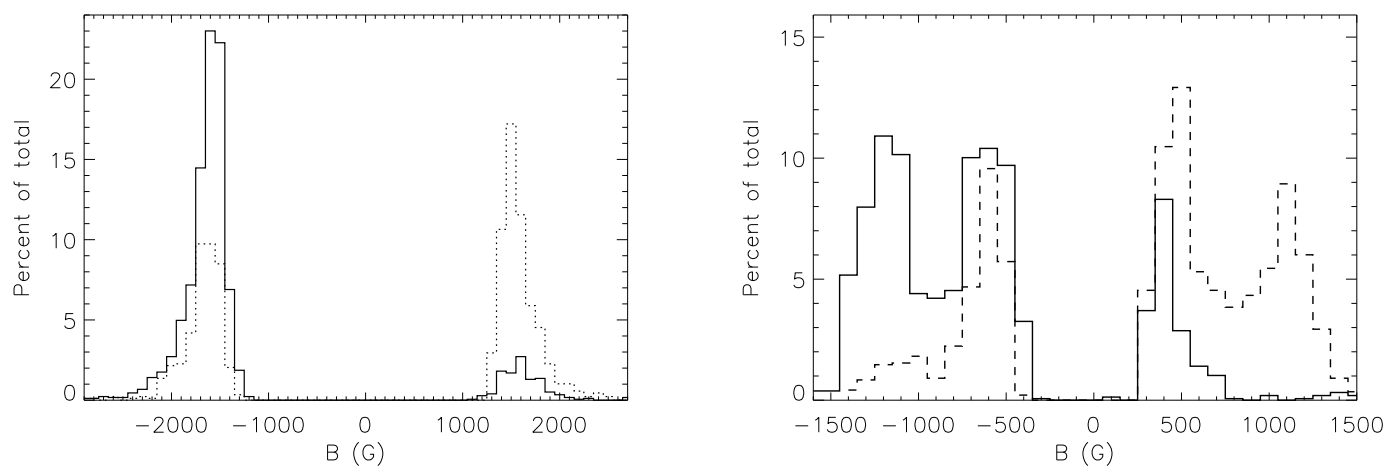

Figure 5.12: Histograms of magnetic field strengths from the separation of Stokes $V$ extrema for FPI data (left) and TIP II data (right). The solid lines correspond to the northern PFe and the dotted and dashed lines to the southern PFe.

The percentage ordinate refers to the PF pixels, separately for the northern and southern polar caps. The left frame corresponds to FPI data and the right frame to TIP II data.

From the visible line observations, the histograms peak at field strengths of 1400$1600 \mathrm{G}$. For the infrared line, the histograms show a bimodal distribution, with the high peak reaching again the kilo-Gauss regime, at 1100-1200 G. This high peak is especially pronounced for PFe of the same polarity as the global magnetic field, whereas for opposite polarity $\mathrm{PFe}$ it is much less clear.

In the case of the visible line measurements, the histograms exhibit tails that extend into stronger fields. This arises from the overestimation of the field strength when applying the SFR, as noted in Sect. 4.3

\subsubsection{Total magnetic flux in PFe}

This section deals with the role of PFe in the global magnetic field of the Sun. From magnetic flux densities in PFe and from the areas of PFe extrapolated from the observed FOVs to the polar cap areas, the total magnetic fluxes from PFe are measured. These total fluxes are then compared with measurements from previous works on PFe and with the global magnetic flux at the solar poles.

As already noted in Sect. 5.1.1 the PF size determination from TIP II data is not as reliable as from FPI data, due to the lower spatial resolution, a key requirement for an accurate measurement. Thus, the estimations for this section are performed using FPI data. For the determination of the total magnetic flux from PFe at the polar caps, a magnetic field strength of $1500 \mathrm{G}$ at the base of PFe is adopted, from the FPI histograms in Fig. 5.12 The areas $F_{\mathrm{PF}}\left(\right.$ in $\left.\mathrm{cm}^{2}\right)$ are taken from Table 5.2. The total magnetic flux is then

$$
\Phi_{\mathrm{PC}}=\alpha \times 1500 \times\left(\sum F_{\mathrm{PF}}\right)[\mathrm{Mx}] .
$$

Here, a magnetic filling gactor $\alpha$ is included to account for the limited spatial resolution. This calculation was done separately for the northern and southern polar caps, for both magnetic polarities. Three different magnetic filling factors of $\alpha=0.1, \alpha=0.3$, and $\alpha=1.0$ were used. In view of the COG measurements of PF magnetic fields (Fig. 5.9) which gave strengths of $\sim 100 \mathrm{G}$ without variation towards the limb, a magnetic filling factor of 
$\alpha=0.1$ appears reasonable. The results are presented in Table 5.4.

Table 5.4: Total magnetic fluxes $\Phi_{\mathrm{PC}}$ at the polar areas of the Sun around the minimum of the sunspot cycle. The three numbers for the entries from this work refer to the fluxes with magnetic filling factor $\alpha=0.1, \alpha=0.3$, and $\alpha=1.0$, from left to right. The signs indicate the polarity.

\begin{tabular}{|c|c|c|c|}
\hline source & \multicolumn{3}{|c|}{ magnetic flux $\Phi_{\mathrm{PC}}\left[10^{22} \mathrm{Mx}\right]$} \\
\hline Sheelev (1966) & \multicolumn{3}{|c|}{1.2} \\
\hline summing over PFe fluxes & & & \\
\hline Svalgaard et al. (1978) & \multicolumn{3}{|c|}{3.2} \\
\hline from direct magnetograms & & & \\
\hline Smith \& Balogh (1995) & \multicolumn{3}{|c|}{2.8} \\
\hline from Ulysses data & & & \\
\hline Benevolenskava (2004) & \multicolumn{3}{|c|}{$\sim 3.0$} \\
\hline \multicolumn{4}{|l|}{ SoHO/MDI magnetograms } \\
\hline \multicolumn{4}{|l|}{ this work } \\
\hline summing over PFe fluxes & $\alpha=0.1$ & $\alpha=0.3$ & $\alpha=1.0$ \\
\hline north pole, signed flux & -0.095 & -0.32 & -0.95 \\
\hline north pole, unsigned flux & 0.115 & 0.38 & 1.15 \\
\hline south pole, signed flux & +0.032 & +0.11 & +0.32 \\
\hline south pole, unsigned flux & 0.052 & 0.17 & 0.52 \\
\hline \multicolumn{4}{|l|}{ summing over non-PFe fluxes } \\
\hline north pole, signed flux & -0.13 & -0.39 & -1.32 \\
\hline north pole, unsigned flux & 0.25 & 0.76 & 2.52 \\
\hline
\end{tabular}

Table 5.4 shows that, with a filling factor $\alpha=0.1$, the summed magnetic fluxes in PFe are lower by factors 30-100 than needed, to account for the fluxes emanating from the polar caps of the Sun. This is in agreement with the estimate by Okunev et al. (2005), who concluded that approximately $2.5 \times 10^{4} \mathrm{PFe}$, many more than observed, would be needed to account for the global magnetic flux at the polar caps. Around the south pole, more flux is "missing" in the PFe than near the north pole. Yet, since the southern polar area with $\psi \leq-60^{\circ}$ was not well observable during the end of August 2005, as noted above in Sect. 5.1.1, the extrapolation from the observations to the polar cap is uncertain for the south pole.

The filling factor 1.0 appears unlikely as much stronger magnetic signals would have been detected. But even with $\alpha=1.0$, the measured total signed fluxes are too low by factors of 3 and 10 for the northern and southern polar caps, respectively.

Total magnetic fluxes in PFe calculated by means of different approaches, from former studies, are also presented in Table 5.4 for comparison with the present work. They are described below. Likewise, the magnetic flux detectable in the observations which does not belong to PFe is also included in Table 5.4. This non-PFe magnetic flux analysis is detailed in Sect. 5.2.3.

Sheeley (1966), via a calibration of the PF flux with equatorial faculae of similar size, obtained a flux of $\Phi_{\mathrm{PF}} \approx 2 \times 10^{20} \mathrm{Mx}$ per facula and arrived, with approximately $60 \mathrm{PFe}$ present during maximum $\mathrm{PF}$ occurrence, at a total flux from a polar cap of 
$\Phi_{\mathrm{PC}} \approx 1.2 \times 10^{22} \mathrm{Mx}$. For comparison, our estimate of the upper limit of the magnetic flux in one PF, assuming a circular shape with a diameter of $725 \mathrm{~km}\left(\equiv 1^{\prime \prime}\right)$ and a field strength of $1500 \mathrm{G}$, gives $\Phi_{\mathrm{PF}} \approx 6.2 \times 10^{18} \mathrm{Mx}$. This is a factor of 30 less flux than Sheeley's (1966) estimate. Note that the area of a PFe with 1" diameter is significantly larger than the usual areas of observed PFe (cf. Fig. 5.3).

According to Svalgaard et al. (1978) the average polar magnetic field is of the order of $6 \mathrm{G}$. With the polar cap area (Eq. 5.1), one obtains a signed magnetic flux of $\Phi_{\mathrm{PC}} \approx$ $2.5 \times 10^{22} \mathrm{Mx}$. From the variation of the average magnetic flux density towards the poles, these authors obtained a total flux $\Phi_{\mathrm{PC}} \approx 3.2 \times 10^{22} \mathrm{Mx}$.

The (signed) magnetic flux in the fast wind, $\Phi_{\mathrm{FW}}$, calculated at $1 A U$ with $B_{\mathrm{rad}} \approx$ $3.5 \mathrm{nT}(=35 \mu \mathrm{G})($ Smith \& Balogh 1995) is

$$
\Phi_{\mathrm{FW}} \approx 3.5 \times 10^{-5} \times 2 \pi(1 A U)^{2} \int_{25^{\circ}}^{90^{\circ}} \cos \psi \mathrm{d} \psi \approx 2.8 \times 10^{22} \mathrm{Mx},
$$

where $A U$ is the astronomical unit $=1.496 \cdot 10^{8} \mathrm{~km}$. Here the integration from $25^{\circ}$ to $90^{\circ}$ takes into account the angular expansion of the solar wind (McComas et al. 2000). The flux in the fast solar wind is consistent with the value given by Svalgaard et al. (1978). For the magnetic flux at $1 A U$ approximately $4500 \mathrm{PFe}$, with flux per PF from our optimum estimate, are needed.

Benevolenskava (2004), from an analysis of SoHO/MDI magnetograms between $78^{\circ}$ and $88^{\circ}$, obtained total unsigned magnetic fluxes for the north polar cap $\Phi_{\mathrm{PC}} \approx 2.7 \times$ $10^{22} \mathrm{Mx}$ and for the south polar cap $\Phi_{\mathrm{PC}} \approx 3.4 \times 10^{22} \mathrm{Mx}$. As noted in Sect. 5.1.1] she pointed out the asymmetry in both poles. However it was not as strong as that identified in the present study regarding FPI observations.

Two further estimates are presented as follows:

1. A measurement of $60 \mathrm{G}$ in three contiguous pixels (as a minimum requirement for a PF identification) at $\mu=0.4$ (as an example) gives after correction for foreshortening of the area, a flux of $3 \times 10^{16} \mathrm{Mx}$. Then assume a magnetic feature which is spread due to limited resolution to a Gaussian field distribution of 0.'5 FWHM from which we measure a maximum field strength of $60 \mathrm{G}$. This structure contains a flux of $\sim 9 \times 10^{16} \mathrm{Mx}$, i.e. a factor of three more than that in the three pixels above. Therefore, it is concluded here that the filling factor $\alpha$ is possibly larger than 0.1.

2. In the present work a total (extrapolated) number of detected PFe per polar cap of 3500 (Table 5.2) has been adopted. The polar areas with $4.1 \times 10^{11} \mathrm{~km}^{2}$ of PFe occurrence contain approximately 500 supergranulation (or chromospheric) network cells (NCs) of $3 \times 10^{4} \mathrm{~km}$ average diameter. This yields 7 detected PFe per NC. Furthermore, an unsigned flux per polar area of $4 \times 10^{22} \mathrm{Mx}$ is assumed, with $25 \%$ of it in flux of magnetic polarity opposite to the global general field at the solar poles (Table 5.4). Thus each NC harbours a flux of $8 \times 10^{19} \mathrm{Mx}$. This flux would be contained in $13 \mathrm{PFe}$ with the above upper flux estimate per PF of $6.2 \times 10^{18} \mathrm{Mx}$. These numerous strong flux features were not identified, they were definitely not present.

Magnetic fields are prone to become intermittent and concentrated flux tubes by convective collapse and to be advected to the borders of granular and supergranular con- 
vection cells (Galloway \& Weiss 1981; Hasan 1985; Vögler et al. 2005). Therefore the picture of small-scale, strong-field flux tubes is retained in the present work as the building blocks for the general, unipolar magnetic field at the solar poles. Mixed-polarity structures are not excluded, yet require sufficient surplus of one polarity over the other. Assuming a magnetic filling factor $\alpha=0.3$ (Table 5.4), the measured flux in PFe is $3-10 \%$ of the net flux in polar areas.

The main conclusion from the magnetic flux results is that the overwhelming part of the total flux is still hidden in smaller flux tubes or bundles of flux tubes with less flux than those seen in PFe with the present resolution. Thus, this suggests that PFe represent the "large-scale" end of a distribution of flux tubes with kilo-Gauss field strength, with increasing number but decreasing magnetic flux towards smaller scales.

\subsubsection{Magnetic flux outside PFe}

Having analysed the flux in PFe, the magnetic flux in the FOVs not contained in PFe will now be discussed. This flux comes from features which do not have any associated brightness in continuum images although their field strength is above the 60 Gauss threshold (see the examples shown in Figs. 4.6 and 5.1). Thus, these features are not counted as PFe but may account for an important amount of magnetic flux in the polar caps.

Table 5.4 includes the magnetic fluxes in non-facular structures, but only for the north pole, due to the observational limitation of the south pole at the time. For their selection, a flux density of $\left|B_{\text {eff }}\right| \geq 60 \mathrm{G}$ within at least three contiguous pixels was also required. Usually, the field strengths (from COG) of the non-facular structures were smaller than $100 \mathrm{G}$ and, on average, their areas a little smaller than those of PFe. From the total flux presented in Table 5.4 these non-facular structures can account for the same amount of magnetic flux as the PFe. Yet here also a filling factor of 1.0 is unrealistic. In this case, it is expected that they would appear as bright structures. Thus the non-facular magnetic structures seen in this study also do not harbour the missing flux necessary to account for the global magnetic field at the poles of the Sun.

\subsection{Velocity}

A statistical study of LOS velocities in PFe was performed, yielding very similar results for both FPI and TIP II observations. First, an analysis of the variation of velocities towards the limb was carried out. Studying separately upflows and downflows, an increase in both of them towards the disc centre was found. In Fig. 5.13 the mean velocities, determined from the COG shifts of the I profiles, of PFe within each FOV are depicted, for FPI data (left) and TIP II data (right). The error bars correspond to the standard deviation of facular velocities within the same FOV, negative velocities are towards the observer, and zero reference velocity refers to the average of all line positions in the FOVs. The dotted straight lines are least square fits to the data. A clear dependence of velocities on $\mu$ is seen, in the sense of increasing blueshift towards the disc centre. On average, there is a surplus of negative velocities, i.e. flows towards the observer. This is interpreted as predominant up-flows in the bright convective elements as the main constituents of faculae (Fig. 4.6). Regarding the flows away from observer, which are also detected, one 

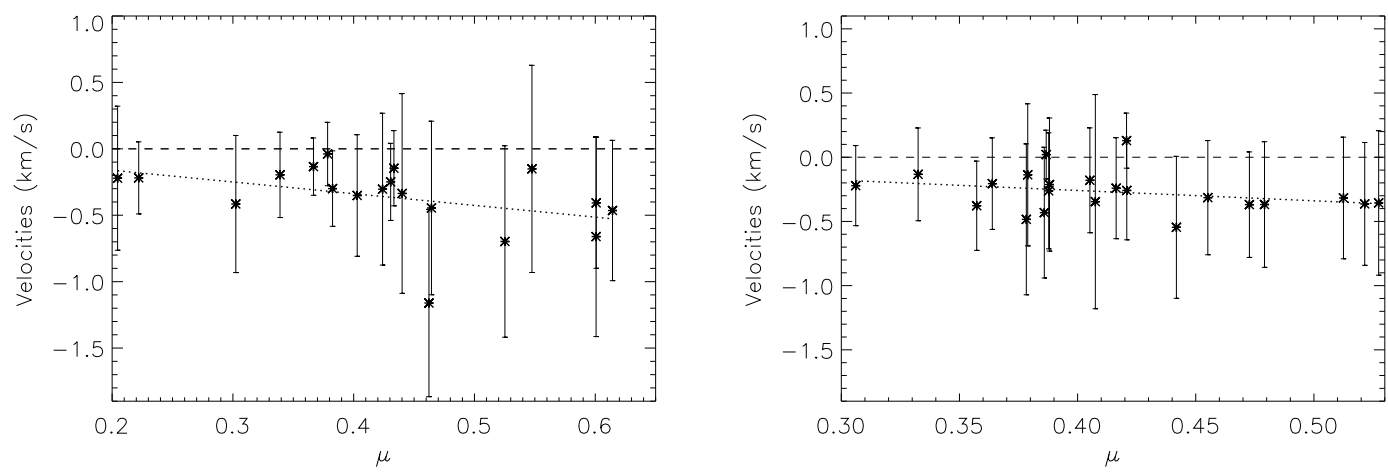

Figure 5.13: Variation of velocities in PFe measured with the COG method from Stokes I profiles for FPI data (left) and TIP II data (right). Asterisks denote the average velocities of PFe in each FOV. Error bars correspond to the standard deviation of facular velocities within the same FOV. Reference zero velocity is the average line position in the FOV.

explanation is that near the limb, at $\mu \leq 0.6$, and depending on the inclination of the convective up- and down-flows, the velocities may also appear in the direction away from the observer.

Second, Figs. 5.14 and 5.15 show separately for PFe near the north and south poles, the velocities measured from all PFe pixels with the COG method applied to the $I$ profiles and with the zero-crossing of the $V$ profiles, for FPI and TIP II observations, respectively. As is also seen in Fig. 5.13, the PF I profiles tend to be blue-shifted, with velocities $\left|v_{\mathrm{COG}}\right|<2.5 \mathrm{~km} \mathrm{~s}^{-1}$. The average COG velocities are $-0.30 \mathrm{~km} \mathrm{~s}^{-1}$ and $-0.61 \mathrm{~km} \mathrm{~s}^{-1}$ at the north and south pole, respectively, for FPI observations. In the case of TIP II data, the values are $-0.29 \mathrm{~km} \mathrm{~s}^{-1}$ and $-0.30 \mathrm{~km} \mathrm{~s}^{-1}$, correspondingly. The velocities from the $V$ zero-crossings are as well spread between $\pm 2 \mathrm{~km} \mathrm{~s}^{-1}$, with averages $\bar{v}_{\mathrm{zc}}=0.15 \mathrm{~km} \mathrm{~s}^{-1}$ and $\bar{v}_{\mathrm{zc}}=-0.29 \mathrm{~km} \mathrm{~s}^{-1}$ for FPI data and $\bar{v}_{\mathrm{zc}}=-0.40 \mathrm{~km} \mathrm{~s}^{-1}$ and $\bar{v}_{\mathrm{zc}}=-0.32 \mathrm{~km} \mathrm{~s}^{-1}$ for TIP II data, at the north and south pole, respectively.

These findings, i.e. a tendency to negative velocities measured in $I$ profiles and, within the measurement accuracy, zero average velocity from $V$ zero-crossings (or slightly negative velocities also for TIP II data), is consistent with the results by Okunev \& Kneer (2004, see their Fig. 11). After adding a velocity due to the gravitational limb effect (Schröter 1957), a net up-flow was found by the latter authors. Okunev et al. (2005) argued that very few PFe with an up-flow of $v_{\mathrm{PF}} \approx 0.5 \mathrm{~km} \mathrm{~s}^{-1}$ would suffice to feed the fast solar wind from the polar coronal holes. In view of the large width of the velocity distributions and in view of the limited accuracy, no further estimates into this direction are developed here, especially since the reference zero velocity in the present observations refers to the average of the line positions in the FOVs, not to the position of the average $I$ profile. This means that the limb effect must not be taken into account in the determination of the zero velocity. For a more accurate analysis, a method of inferring the properties of PF flows is to compare the observed velocities with numerical magneto-hydrodynamic simulations of facular magnetic flux tubes and bundles of flux tubes.

$\mathbf{H} \alpha$ velocities. Once the velocities of $\mathrm{PFe}$ at a photospheric level had been analysed, studying the plasma flow at higher atmospheric layers could give a clue whether $\mathrm{PFe}$ are 

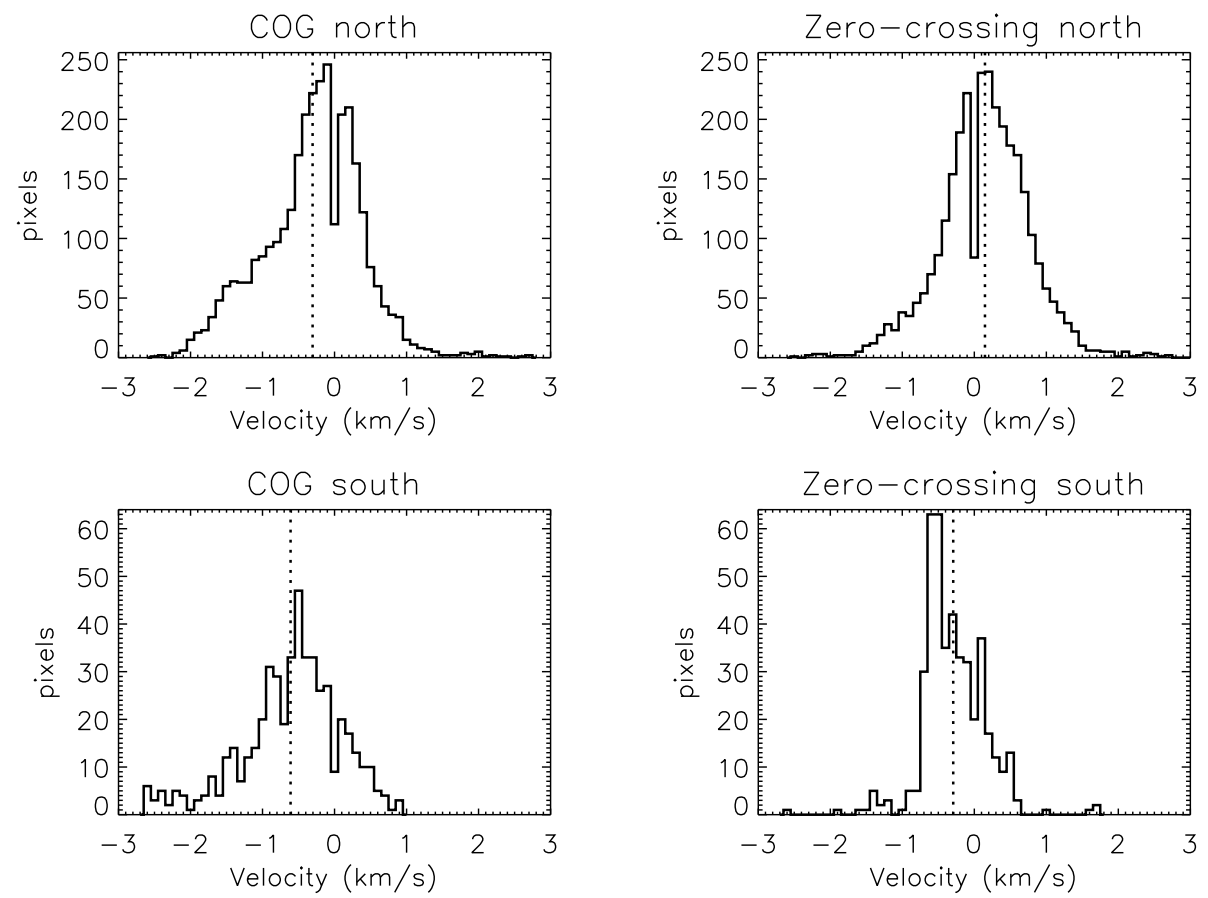

Figure 5.14: Velocities measured from Doppler shift of Stokes $I$ COG and from $V$ zero-crossing from FPI data. Reference zero velocity is the average of the line positions in the FOVs. Vertical dotted lines indicate the average velocities. Positive velocities are away from the observer.
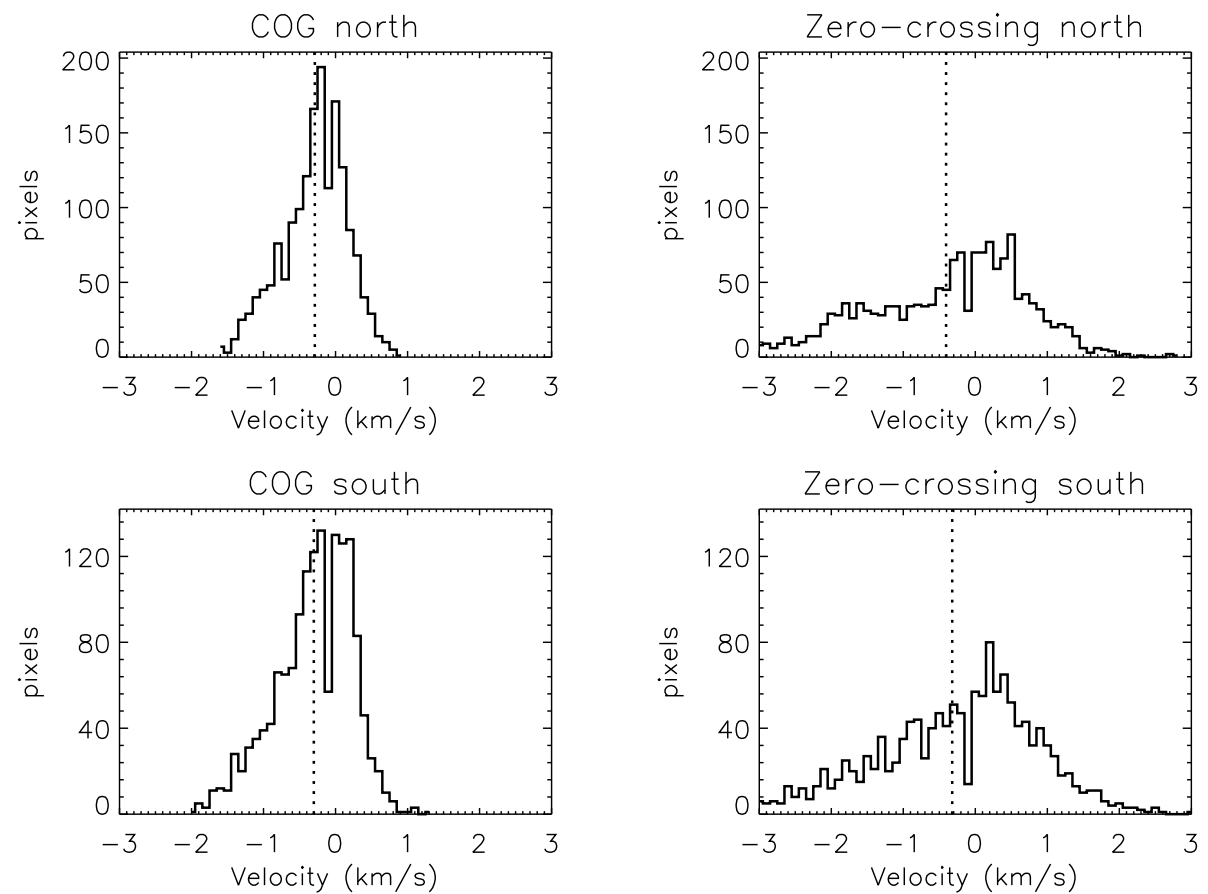

Figure 5.15: Same as Fig. 5.14 for TIP II data. 
feeding the solar wind or the material flow does not reach the outer solar atmosphere. $\mathrm{H} \alpha$ observations were only taken at the northern solar pole, so no information from the south pole is available. The results of the lambdameter method are depicted in Fig. 5.16

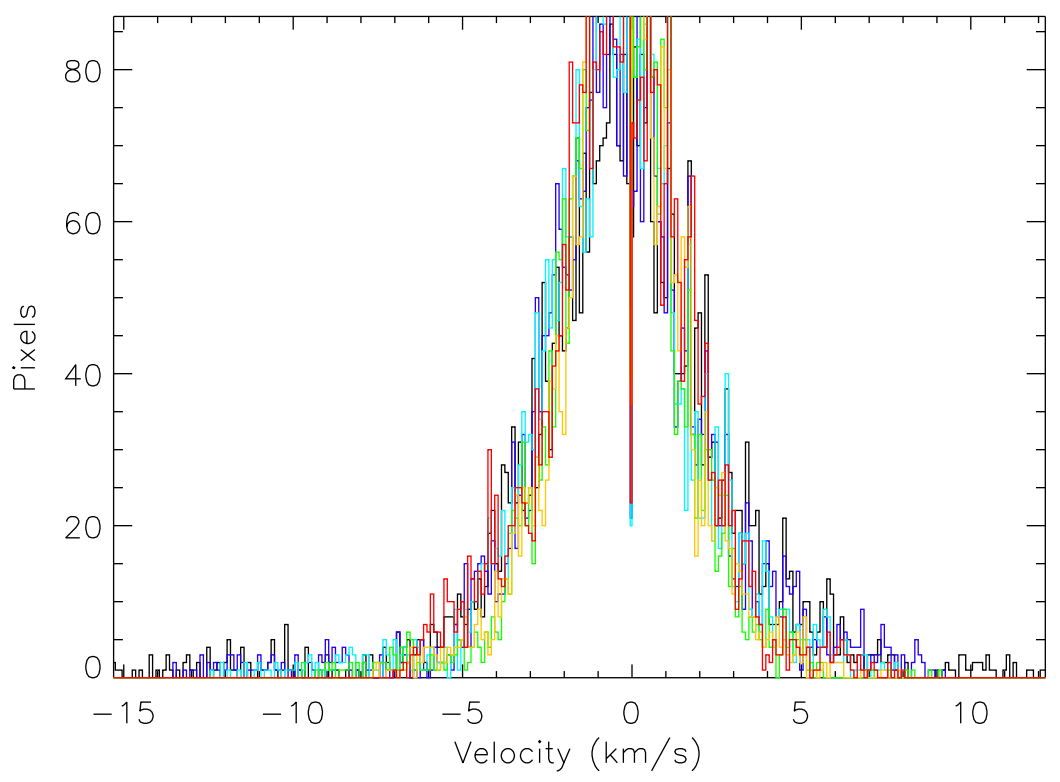

Figure 5.16: Histograms of $\mathrm{H} \alpha$ velocities calculated with the lambdameter method. Colours refer to different wavelength separations for the measurement of the Dopplershift in H $\alpha$, black: $\Delta \lambda=0.2 \AA$, dark blue: $\Delta \lambda=0.41 \AA$, light blue: $\Delta \lambda=0.62 \AA$, green: $\Delta \lambda=0.82 \AA$, orange: $\Delta \lambda=1.03 \AA$, red: $\Delta \lambda=1.23 \AA$. Points with zero velocity have been removed for clarity.

as histograms of the number of pixels for each velocity. The pixels selected to enter the measurement are only those which, at photospheric level, belong to PFe according to the selection criteria described above. The different histograms overplotted as coloured lines correspond to the velocities measured at different heights.

As can be seen in Fig. 5.16, the shapes of the histograms are mostly the same for the six different heights at which velocities were measured in the $\mathrm{H} \alpha$ profile (note that wavelength separations $\Delta \lambda$ for the lambdameter method refer to different heights in the solar atmosphere where the velocity signals are formed). The mean velocities at each height vary slightly between $-0.26 \mathrm{~km} \mathrm{~s}^{-1}$ to $-0.46 \mathrm{~km} \mathrm{~s}^{-1}$, with positive velocities meaning again flows away from the observer. The velocity range from maximum to minimum increases with the atmospheric height from -7 and $+8 \mathrm{~km} \mathrm{~s}^{-1}$ at the lower layer to -15 and $+12 \mathrm{~km} \mathrm{~s}^{-1}$ at the highest layer.

The consistency of the mean velocities obtained from the three different analyses performed here is remarkable. Both photospheric lines, infrared and visible, and the chromospheric $\mathrm{H} \alpha$ line indicate a low although clear continuous outflow of material from $\mathrm{PFe}$ from the photosphere towards higher layers. This suggests that the plasma feeding the solar wind could indeed come from PFe.

From magnetic field extrapolation and data from the SUMER instrument on board the SoHO spacecraft, Tu et al. (2005) suggested that plasma outflows are accelerated inside coronal funnels at a height of $5 \mathrm{Mm}$. The analyses performed in the present work reach 
a height of approximately $1 \mathrm{Mm}$. Observations of PFe at higher layers than $1 \mathrm{Mm}$ could confirm the scenario presented by Tu et al. (2005) and the continuous upflow of material and eventual feeding of the fast solar wind from PFe. 


\title{
6 Conclusions
}

\author{
"... a four-year-old child could understand this report. \\ Run out and find me a four-year-old child, I can't make head or tail of it." \\ Duck Soup (1933)
}

This thesis work has focused in the analysis of polar faculae (PFe) on the Sun, both to have a more in-depth characterisation of these structures and to understand their significance on a global scale.

State-of-the-art observations with recently upgraded instrumentation have been the cornerstone for this study. Data of high spectral resolution with high polarimetric sensitivity observed in the magnetic infrared lines at $1.56 \mu$ have been combined with data with very high spatial resolution in the photospheric visible line of $\mathrm{Fe}_{\mathrm{I}} 6173 \AA$ and the chromospheric $\mathrm{H} \alpha$ line to investigate $\mathrm{PFe}$ at different wavelength ranges and different atmospheric layers on the Sun. Measurements of properties with the visible and infrared magnetic lines have yielded very similar results, giving consistency to the analyses.

The observations for this work were performed when the Sun was near a minimum of sunspot activity, i.e. the global magnetic field was mostly poloidal and the occurrence of PFe was maximum. Thanks to this, statistical analyes of PFe from a wide range of heliocentric angles have been performed.

Spatial resolution has been proved to be the most important constraint in the study of these small-scale features. Area estimations and contrast measurements of PFe are highly affected by the spatial resolution of the observations, in the sense that, with lower spatial resolution, $\mathrm{PF}$ areas appear larger (since PFe cannot be discerned into their smaller components) and contrasts are lower than with high spatial resolution. This effect, regarding the contrast measurements, increases with decreasing distance to the solar limb of the observations.

A much higher number of PFe than hitherto observed was found in the observations for this work. Probably with even higher resolution than the one achieved here, PFe can be resolved into smaller components and still higher numbers can be counted.

From a centre-to-limb study of the occurrence of PFe a step was found at $\mu \approx 0.4$ in both visible and infrared lines. A bias introduced in the PF selection process by either the intensity threshold or the magnetic field threshold is to be discarded. Presumably, the drop in the PF numbers can be explained by a projection effect. With increasing heliocentric angles interfacular areas become hidden and individual faculae appear then forming part of a larger structure. Again, observations with higher spatial resolution can help in studying this possibility. Also observations from out-of-ecliptic spacecraft, with better views to the poles of the Sun, will not have this projection problem. 
Throughout the PF counting, PFe with opposite magnetic polarity than that of the global field were found in higher numbers than thought previously. Most PFe possess a magnetic field with the same polarity as the global field. But the opposite polarity faculae amount up to $20-30 \%$ of the total PF number, making them a non-negligible phenomenon.

Besides, PFe with the same polarity as that of the global field tend, on average, to be larger in size than those with opposite polarity.

To study the impact of PFe on a solar global scale, extrapolations of the number and sizes of PFe were performed from the areas observed during the realisation of this work to the total polar cap areas. When comparing the occurrence of PFe near each solar pole, an important asymmetry was found. The north pole harbours, at the dates of the observations, a larger number of PFe than the south pole. The asymmetry is not so strong for the infrared line observations with respect to the visible line. However, at the time of the visible line observations, the south pole was not so easily observable due to the inclination of the solar axis with respect to the ecliptic pole.

The PF contrast was measured from speckle reconstructed images and its centre-tolimb variation analysed and compared with that of the brightest granules in the vicinity of the PFe. At the lower boundary of PF appearance, the contrast of both PFe and brightest granules converge to a common value. Thus, at $\mu>0.6 \mathrm{PFe}$ are very difficult to distinguish in intensity from granules. Towards limb, a steep increase in the PF contrast until $\mu \approx 0.4$ turns to an approximately constant value for higher heliocentric angles, i.e. for smaller $\mu$.

Quasi-simultaneous observations of the magnetically sensitive $6173 \AA$ iron line and $\mathrm{H} \alpha$ allow to investigate the penetration of PFe into the chromosphere and the response of $\mathrm{H} \alpha$ as a magnetic field proxy. $\mathrm{H} \alpha$ has proved to be a reliable proxy for magnetic bright elements when observed in the blue wing, at -0.7 to $-0.8 \AA$ off line minimum. Starting from the blue wing towards the line centre - i.e. with increasing height in the chromosphere -, PFe brightenings in $\mathrm{H} \alpha$ seem to expand from the centre of PFe. A full comprenhension of $\mathrm{H} \alpha$ observations falls outside the scope of this thesis work. Future analyses will focus on the behaviour of PFe in the chromospheric layer.

High spatial resolution speckle reconstructed images have proven that smaller components of PFe evolve in time scales of the order of 10 seconds, whereas big PF structures remain present for several hours. Future studies should aim to measure PF magnetic fields in these short time scales and at this high spatial resolution to match the intensity analyses.

The line of sight component of the magnetic field in the visible spectral line and the LOS and transversal components of the magnetic field in the infared line were measured and their relation with the heliocentric angle analysed. All of them show a common result: No apparent variation of the components towards limb is present. Instrumental cross-talk of polarisation signals, that possibly falsify the magnetic field measurements, and a bias from selection thresholds are found not to be responsible and are discarded. Thus, this puzzling result needs further studies for understanding.

In the case of the LOS component of the field measured with the centre of gravity method, the magnetic field retrieved from infrared observations is approximately four times lower than that in the visible observations. However, the separations of Stokes $V$ extrema in the infrared lines indicate that a higher strength should be obtained. The reason for this difference is the lower spatial resolution of the infrared observations. As a consequence, 
a larger non-magnetic area affects the profiles from the magnetic areas and the centre of gravity method retrieves a lower magnetic field.

From histograms of total magnetic field strength, PFe possess, as noted in previous studies, a magnetic field strength in the kilo-Gauss range, of approximately $1500 \mathrm{G}$.

The contribution of PFe to the global magnetic flux was calculated by extrapolation of $\mathrm{PF}$ areas and considering a magnetic field strength per PF of $1500 \mathrm{G}$. With these values and, even assuming a magnetic filling factor $\alpha=1.0$, the total magnetic flux in PFe is 3 to 10 times lower than that needed to account for the polar magnetic flux obtained by other methods in earlier studies.

Likewise, the total magnetic flux found in the observations not contained in PFe, i.e. seated in magnetic structures with no associated continuum brightness, is too low to account for the magnetic flux from the polar caps measured with other methods.

Velocities in PFe were measured in the three different regimes treated in this work. All of them, infrared, visible photospheric, and chromospheric, give very similar results: a small upflow of material with a velocity of approximately $0.3 \mathrm{~km} \mathrm{~s}^{-1}$. This upflow of material suggests PFe as candidates for the photospheric sources of the fast solar wind. Observations from higher atmospheric layers are needed to confirm the continuous upflows until the acceleration region of the solar wind.

Alternatively, the measured net upflows could be the result of magnetoconvection. Possibly one observes shifts of Stokes $I$ and $V$ profiles as a complex phenomenon of convection seen obliquely (near the limb) through semi-transparent magnetised gas. Numerical simulations of magnetoconvection and of the expected polarised light from PFe are needed to study this possibility. 



\section{Bibliography}

"Gentlemen, gentlemen... All these books, a world of knowledge at your fingertips, and you play poker all night."

Seven (1995)

Al, N., Bendlin, C., Hirzberger, J., Kneer, F., \& Trujillo Bueno, J. 2004, A\&A, 418, 1131

Axford, W. I., \& McKenzie, J. F. 1997, in Cosmic Winds and the Heliosphere, ed. J. R. Jokipii, C.- P. Sonett, \& M. S. Giampapa, Arizona University Press, Tucson, p. 31

Auffret, H., \& Muller, R. 1991, A\&A, 246, 264

Baumann, I., Schmitt, D., Schüssler, M., \& Solanki, S. K. 2004, A\&A, 426, 1075

Baumann, I., Schmitt, D., \& Schüssler, M. 2006, A\&A, 446, 307

Beckers, J. M. 1964, PhD Thesis, Utrecht

Bello González, N., Okunev, O. V., Domínguez Cerdeña, I., Kneer, F., \& Puschmann, K. G. 2005, A\&A, 434, 317

Bello González, N. 2006, PhD Thesis, Göttingen

Bello González, N., \& Kneer, F. 2008, A\&A, in press

Bendlin, C. 1993, PhD thesis, Göttingen University

Bendlin, C., \& Volkmer, R. 1993, A\&A, 278, 601

Bendlin, C., \& Volkmer, R. 1995, A\&AS, 112, 371

Bendlin, C., Volkmer, R., \& Kneer, F. 1992, A\&A, 257, 817

Benevolenskaya, E. E. 2004, A\&A, 428, 5

Biermann, L. 1951, Z. Astrophys., 29, 274

Blanco Rodríguez, J., Okunev, O., Puschmann, K. G., Kneer, F., \& Sánchez-Andrade Nuño, B. 2007, A\&A, 474, 251 
Brault, J. W., \& Neckel, H. 1987 Spectral Atlas of Solar Absolute Disk-Averaged and Disk-Centre Intensity from $3290 \AA$ to $12510 \AA$

Collados, M. 1999, 3rd Advances in Solar Physics Euroconference: Magnetic fields and Oscillations, ed. Schmieder, B., Hofmann, A., Staude, J., ASP Conf. Ser., 184, 3

Collados Vera, M., Lagg, A., Díaz García, J. J., Hernández Suárez, E., López López, R., Páez Mañá, E., \& Solanki, S. K. 2007, in The Physics of Chromospheric Plasmas, ed. P. Heinzel, I. Dorotovič, \& R. J. Rutten, ASP Conf. Ser., 368, 611

de Boer, C. R. 1996, A\&AS, 120, 195

DeForest, C. E., Hoeksema, J. T., Gurman, J. B., et al. 1997, Sol. Phys., 175, 393

De Pontieu, B., Carlsson, M., Stein, R., Rouppe van der Voort, L., Löfdahl, M., van Noort, M., Nordlund, Å, \& Scharmer, G. 2006, ApJ, 646, 1405

del Toro Iniesta, J. C. 2003, Introduction to Spectropolarimetry, Cambridge University Press

Delaboudinière, J.-P., Artzner, G. E., Brunaud, J., et al. 1995, Sol. Phys., 162, 291

Domingo, V., Ortiz, A., Sanahuja, B., \& Cabello, I. 2005, Adv. Space Res, 35, 345

Fabiani Bendicho, P., Kneer, F., \& Trujillo Bueno, J. 1992, A\&A, 264, 229

Fried, D. L. 1966, J. Opt. Soc. Am., 56, 1372

Galloway, D. J., \& Weiss, N. O. 1981, ApJ, 243, 945

Hasan, S. S. 1985, A\&A, 151, 69

Homann, T., Kneer, F., \& Makarov, V. I. 1997, Sol. Phys., 175, 81

Hirzberger, J., \& Wiehr, E. 2005, A\&A, 438, 1059

Keller, C. U., \& von der Lühe, O. 1992, A\&A, 261, 321

Keller, C. U., Schüssler, M., Vögler, A., \& Zakharov, V. 2004, ApJ, 607, L59

Kneer, F., \& Hirzberger, J. 2001, AN, 322, 375

Korff, D. 1973, J. Opt. Soc. Am., 63, 971

Koschinsky, M. 2001, PhD thesis, Göttingen University

Koschinsky, M., Kneer, F., \& Hirzberger, J. 2001, A\&A, 365, 588

Labeyrie, A. 1970, A\&A, 6, 85

Landi Degl'Innocenti, E. 1992, in Solar Observations: Techniques and Interpretation, First Canary Islands Winter School, ed. F. Sánchez, M. Collados, \& M. Vázquez, Cambridge Univ. Press, Cambridge UK, p.73 
Lawrence, J. K., \& Chapman, G. A. 1988, ApJ, 335, 996

Leenaarts, J., Rutten, R. J., Sütterlin, P., Carlsson, M., \& Uitenbroek, H. 2006, A\&A, 449, 1209

Lin, H., Varsik, J., \& Zirin, H. 1994, Sol. Phys., 155, 243

Livingston, W., \& Wallace, L. 1991, NSO Technical Report, Tucson: National Solar Observatory, National Optical Astronomy Observatory or NSO Technical Report, 91001

Makarov, V. I., \& Sivaraman, K. R. 1989, Sol. Phys., 123, 367

Makarov, V. I., Makarova, L. V., Bogod, V. N., et al. 1991, Soln. Dann. 9, 87

Makarov, V. I., \& Makarova, L. V. 1996, Solar Phys., 163, 267

Makarov, V. I., Tlatov, A. G., \& Sivaraman, K. R. 2003a, Astron. Nachr./AN, 324, 382

Makarov, V. I., Tlatov, A. G., \& Sivaraman, K. R. 2003b, Sol. Phys., 214, 41

Martínez Pillet, V. 1997, Optical Design of a visible polarimeter, IAC internal report

Martínez Pillet, V., Collados, M., Sánchez Almeida, J., Gónzalez, V., Cruz-Lopez, A., Manescau, A., Joven, E., Paez, E., Diaz, J. J., Feeny, O., \& Sánchez, V. 1999, High Resolution Solar Physics: Theory, Observations, and Techniques, ed. Rimmele, T. R., Balasubramaniam, K. S., \& Radick, R. R., ASP Conf. Ser., 183, 264

McComas, D. J., Barraclough, B. L., Funsten, H. O., et al. 2000, J. Geophys. Res., 105(A5), 10419

Neckel, H. 1999, Sol. Phys., 184, 421

Okunev, O. V. 2004, PhD thesis, Göttingen University

Okunev, O. V., \& Kneer, F. 2004, A\&A, 425, 321

Okunev, O. V., \& Kneer, F. 2005, A\&A, 439, 323

Okunev, O. V., Domínguez Cerdeña, I., Puschmann, K. G., Kneer, F., \& Sánchez Almeida, J. 2005, Astron. Nachr./AN, 326, 205

Ortiz, A., Solanki, S. K., Domingo, V., Fligge, M., \& Sanahuja, B. 2002, A\&A, 388, 1036

Pehlemann, E., \& von der Lühe, O. 1989, A\&A, 216, 337

Penza, V., Caccin, B., Ermolli, I., \& Centrone, M. 2004, A\&A, 413, 1115

Puschmann, K. G., Kneer, F., Seelemann, T., \& Wittmann, A. D. 2006, A\&A, 451, 1151

Puschmann, K. G., \& Sailer, M. 2006, A\&A, 454, 1011

Rees, D. E., \& Semel, M. D. 1979, A\&A, 74, 1 
Rees, D. E., Murphy, G. A., \& Durrant, C. J.: 1989, ApJ, 339, 1093

Riehokainen, A., Urpo, S., \& Valtaoja, E. 1998, A\&A, 333, 741

Riehokainen, A., Urpo, S., Valtaoja, E., Makarov, V. I., Makarova, L. V., \& Tlatov, A. G. 2001, A\&A, 366, 676

Sailer, M. J. 2006, PhD thesis, Göttingen University

Sánchez Almeida, J., Collados, M., \& Martínez Pillet, V. 1994, Modulation schemes for the polarimeter of the SVST, IAC internal report

Sánchez Cuberes, M., Bonet, J. A., Vázquez, M., \& Wittmann, A. D. 2000, A\&A, 538, 940

Scherrer, P. H., Bogart, R. S., Bush, R. I., et al. 1995, Sol. Phys., 162, 129

Schröter, E. H. 1957, Zeitschrift für Astrophysik, 41, 141

Schüssler, M., \& Rempel, M. 2005, A\&A, 441, 337

Semel, M. D. 1967, Ann. Astrophys., 30, 513

Sheeley, N. R., Jr. 1964, ApJ, 140, 731

Sheeley, N. R., Jr. 1966, ApJ, 144, 723

Sheeley, N. R., Jr. 1991, ApJ, 374, 386

Shurcliff, W. A. 1962, Polarized Light, Harvard University Press, Cambridge

Smith, E. J., \& Balogh, A. 1995, Geophys. Res. Lett., 22, 3317

Solanki, S. K., \& Fligge, M. 2002, Adv. Space Res. 29, 1933

Solonski, Yu. A., \& Makarova, V. V. 1992, Sol. Phys., 139, 233

Spruit, H. C. 1976, Sol. Phys., 50, 269

Steiner, O. 2007, in Modern Solar Facilities - Advanced Solar Science, ed. F. Kneer, K. G. Puschmann, \& A. D. Wittmann, publisher Universitätsverlag Göttingen, p. 321

Svalgaard, L., Duvall, T. L., Jr., \& Scherrer, P. H. 1978, Sol. Phys., 58, 225

Tsiropoula, G., Alissandrakis, C. E., \& Schmieder, B. 1993, A\&A, 271, 574

Tu, C.-Y., Zhou, C., Marsch, E., et al. 2005, Science, 308, 519

Vernazza, J. E., Avrett, E. H., \& Loeser, R. 1981, ApJS, 45, 635

von der Lühe, O. 1984, J. Opt. Soc. Am. A1, 510

von der Lühe, O., Soltau, D., Berkefeld, T., \& Schelenz, T. 2003, SPIE, 4853, 187 
Vögler, A., Shelyag, S., Schüssler, M., et al. 2005, A\&A, 429, 335

Volkmer, R. 1995, PhD thesis, Göttingen University

Waldmeier, M. 1955, ZAp, 38, 37

Waldmeier, M. 1962, ZAp, 54, 260

Wang, Y.-M., \& Sheeley, N. R., Jr. 2003, ApJ, 599, 1404

Wang, Y.-M., Sheeley, N. R., Jr., \& Lean, J. 2002, ApJ, 580, 1188

Weigelt, G. P. 1977, Optics Comm., 21, 55

Wiegelmann, T., Xia, L. D., \& Marsch, E. 2005, A\&A, 432, L1

Wilhelm, K., Curdt, W., Marsch, E., et al. 1995, Sol. Phys., 162, 189

Wilhelm, K., Marsch, E., Dwivedi, B. N., et al. 1998, ApJ, 500, 1023

Wilhelm, K., Dammasch, I. E., Marsch, E., \& Hassler, D. M. 2000, A\&A, 353, 749

Xia, L. D., Marsch, E., \& Wilhelm, K. 2004, A\&A, 424, 1025

Yi, Z., \& Molowny Horas, R. L. 1992, LEST Technical Report 56, ed. O. Engvold, \& Ø. Hauge, Institute of Theoretical Astrophysics, Oslo University, 69 



\section{Publications}

"Truly, for some men nothing is written unless they write it."

Lawrence Of Arabia (1962)

Blanco Rodríguez, J., Okunev, O., Puschmann, K. G., Kneer, F., Sánchez-Andrade Nuño, B.: On the properties of faculae at the poles of the sun, 2007, A\&A, 474, 251

Sánchez-Andrade Nuño, B., Centeno, R., Puschmann, K. G., Trujillo Bueno, J., Blanco Rodríguez, J., Kneer, F.: Spicule emission profiles observed in He I 10830 A , 2007, A\&A, 472, L51

Sánchez-Andrade Nuño, B., Bello González, N., Blanco Rodríguez, J., Kneer, F., Puschmann, K. G.: Fast events and waves in an active region of the Sun observed in $\mathrm{H \alpha}$ with high spatial resolution, 2008, A\&A, submitted

Blanco Rodríguez, J., Okunev, O., Puschmann, K. G., Kneer, F.: Study of polar faculae with north pole coverage of the Sun, 2007, in Modern solar facilities - Advanced Solar Science, ed. F. Kneer, K. G. Puschmann, A. D. Wittmann, publisher Universitätsverlag Göttingen

Blanco Rodríguez, J., Sánchez-Andrade Nuño, B., Puschmann, K. G., Kneer, F.: Study of Polar Faculae, 2005, in International Scientific Conference on Chromospheric and Coronal Magnetic Fields, ed. D.E. Innes, A. Lagg, S.K. Solanki, on CD-Rom

Sánchez-Andrade Nuño, B., Puschmann K. G., Sánchez Cuberes, M., Blanco Rodríguez, J., Kneer, F.: Analysis of a Wide Chromospheric Active Region, 2005, in International Scientific Conference on Chromospheric and Coronal Magnetic Fields, ed. D.E. Innes, A. Lagg, S.K. Solanki, on CD-Rom

Kneer, F., Puschmann, K. G., Blanco Rodríguez, J., Sánchez-Andrade Nuño, B., Wittmann, A. D.: Magnetic Structures on the Sun: Osbervations with the New "GÖTTINGEN" TwoDimensional Spectrometer on Tenerife, 2005, in International Scientific Conference on Chromospheric and Coronal Magnetic Fields, ed. D.E. Innes, A. Lagg, S.K. Solanki, on CD-Rom

Blanco Rodríguez, J., Sánchez-Andrade Nuño, B., Puschmann, K. G., Kneer, F.: Study of Polar Faculae, 2005, in 11th European Solar Physics Meeting "The Dynamic Sun: 
Challenges for Theory and Observations", ed. D. Danesy, S. Poedts, A. De Groof and J. Andries, on CD-Rom

Sánchez-Andrade Nuño, B., Puschmann K. G., Sánchez Cuberes, M., Blanco Rodríguez, J., Kneer, F.: Chromospheric Dynamics of a Solar Active Region, 2005, in 11th European Solar Physics Meeting "The Dynamic Sun: Challenges for Theory and Observations", ed. D. Danesy, S. Poedts, A. De Groof and J. Andries, on CD-Rom 


\section{Acknowledgements}

"Only dream I ever have... is the surface of the Sun... every time I shut my eyes"

Sunshine (2007)

First of all, I must thank my parents. They give me the opportunity to study Physics, and to go to Teneriffe to complete Astrophysics. They have had the courage to consider the happiness of their son over their doubts and preferences. They have always been there, always supporting, always encouraging and worrying, always loving. To them and the rest of my family, thanks.

The german word for supervisor is Doktorvater, and Franz deserves indeed that title for he has been more a father than just a supervisor. Ever taking care of his students, pushing us into the right way, cheering us and having time for everything, I cannot imagine these past years with other supervisor. This thesis would have been a piece of hot hell without him.

Academically, I cannot avoid acknowledging the teachers who showed and opened this path for me: Mercedes who was an amazing tutor and who is an incredible teacher and better person; Inés with who I had my first contact with real astrophysics back in La Gomera ten years ago, and who was also my first reference in Teneriffe, apart of one of the best communicators and persons I have met; Basilio who taught me three different subjects and managed to make each of them even more fascinating than the previous, who is a source of advices and good mood; Fernando who made the experience of being his student an amazing one and was always helpful despite the little time he has. And some others that remain unwritten in the inker.

Many thanks must be given to Rebecca Centeno and Manuel Collados because of their inestimable help in the calibration and use of TIP II. Many more for their nice conversations, explanations and willingness to help at any time.

Thanks to the Kiepenheuer Institut in Freiburg, which operates the VTT where all the observations for this thesis were realized, especially to Thomas Keller and Joe Staiger who were always ready to help and take care.

I must also thank my institute partners here at IAG for some nice talks about science and about non-work related things. I want to thank especially all my mates of the solar 
group which help me so much during the realisation of this work, both in the academical view and in the social one. I will always remember the teeminars. Thanks to Naza and Benoit, who have taken care of me from the very beginning, and still are at it. And thanks to Klaus for his help and company during the Göttigen months. Oleg was the best guide to this PFe world, and Markus and Bruno deserve a part of this thesis also, take it. Uli Duenzig has been with me in many observational campaigns and his help and good mood are always needed.

Thanks to the DFG for the grant which allowed me to complete this work and to the IAC for considering me worthy. Thanks also to the Max Planck Institute in Lindau for the last months support and for the nice enviroment provided. Many more thanks to the people there who always put a smile in the Sun's face and another one in mine.

I need to thank also my other families, those friends who, despite time past, meet me whenever we can and are still not fed up with me. Their laughs, support and love are a great part of what makes life bearable. Lucky me, there are too many names to be written here.

Mis niñas in Salamanca who look after me more than I deserve.

The gang from high-school with so many years and table footballs at our backs.

The fisicastros from Salamanca who were (and are) the ideal partners to survive that hell. Por Zkeletor!

The crazy astrophysicists from La Laguna, spreading and taking over the world. Still waiting for the Gualtrapillas Astrophysical Journal. Por Crom!

The people met in Göttingen from all around the world sharing glühweins, residence and whiskyseminars. Culture always on top.

And more and more people: Anita, Raquel, ...

Finally, thanks also to Bill Watterson and Matt Groening whose creations have become part of me more than I dare to accept and manage to cheer me up no matter what.

To all of you, and those who have touched me in some way or another along the years, a part of this thesis is because of you. Gracias!

May the road rise to meet you.

May the wind be always at your back.

May the Sun shine warm upon your face.

And rains fall soft upon your fields.

And until we meet again,

May God hold you in the hollow of His hand.

(Irish blessing)

And now, for something completely different... 


\section{Curriculum Vitae}

Name: $\quad$ Julián Blanco Rodríguez

Geburtsdatum: $\quad 14.05 .1980$

Geburtsort: $\quad$ Salamanca, Spanien

Familienstand: $\quad$ ledig

Eltern: Julián Blanco Pollo

María Rodríguez Artigas

Staatsangehörigkeit: Spanisch

Schulbildung: September 1986 - Juni 1998 Colegio "Maristas Champagnat" (Salamanca)

Studium: $\quad$ September 1998 - Juli 2002 Physikalische Fakultät

der Universität Salamanca

September 2002 - Juli 2004 Physikalische Fakultät

der Universität La Laguna

Proyecto fin de carrera:

"Estudio de datos heliosísmicos sintéticos"

Promotion: Oktober 2004 - Januar 2008 Promotion an der Universitäts-Sternwarte, Göttingen (seit Juni 2005 Institut für Astrophysik Göttingen, IAG)

Stipendium der Deutschen Forschungsgemeinschaft 418 SPA-112/15/04

und der International Max Planck Research

School on Physical Processes in the Solar

System and Beyond 